\title{
Time scales for Majorana manipulation using Coulomb blockade in gate-controlled superconducting nanowires
}

\author{
Michael Hell ${ }^{(1,2)}$, Jeroen Danon ${ }^{(2,3)}$, Karsten Flensberg ${ }^{(2)}$, and Martin Leijnse ${ }^{(1,2)}$ \\ (1) Division of Solid State Physics and NanoLund, \\ Lund University, Box. 118, S-22100, Lund, Sweden \\ (2) Center for Quantum Devices and Station $Q$ Copenhagen, \\ Niels Bohr Institute, University of Copenhagen, Copenhagen, Denmark \\ (3) Niels Bohr International Academy, Niels Bohr Institute, \\ University of Copenhagen, Copenhagen, Denmark
}

(Dated: March 5, 2018)

\begin{abstract}
We numerically compute the low-energy spectrum of a gate-controlled superconducting topological nanowire segmented into two islands, each Josephson-coupled to a bulk superconductor. This device may host two pairs of Majorana bound states and could provide a platform for testing Majorana fusion rules. We analyze the crossover between (i) a charge-dominated regime utilizable for initialization and readout of Majorana bound states, (ii) a single-island regime for dominating inter-island Majorana coupling, (iii) a Josephson-plasmon regime for large coupling to the bulk superconductors, and (iv) a regime of four Majorana bound states allowing for topologically protected Majorana manipulations. From the energy spectrum, we derive conservative estimates for the time scales of a fusion-rule testing protocol proposed recently arXiv:1511.05153. We also analyze the steps needed for basic Majorana braiding operations in branched nanowire structures.
\end{abstract}

PACS numbers: 71.10.Pm, 74.50.+r, 68.65.La

\section{INTRODUCTION}

Systems with topologically nontrivial phases have become a focal point of condensed-matter research over the past decade [1] and especially systems hosting Majorana bound states (MBS) [2] have been heavily pursued [38. Two MBS can form a fermionic mode, which can be occupied at the cost of zero energy, that is, the ground state has a fermion-parity degeneracy. This degeneracy is topologically protected against perturbations, which implies that MBS obey non-Abelian exchange statistics [9]. Hence, exchanging MBS (braiding) changes the groundstate in a nontrivial way, a key ingredient for topological quantum computation [10 14].

Identifying a suitable platform for realizing and manipulating MBS, however, remains challenging. MBS exist only in superconductors with triplet pairing [15, which appears intrinsically in $\mathrm{Sr}_{2} \mathrm{RuO}_{4}$ [16 or can be induced extrinsically as a proximity effect [17]. The first candidate systems for MBS were vortices of $2 \mathrm{D}$ triplet superconductors, in which the MBS might be manipulated through gate-voltage controlled point contacts 18 or supercurrents [19]. As an arguably more feasible alternative, 1D systems have been considered $20+22$ and among those magnetic atom chains 23 and semiconductor nanowires [24, 25] have been suggested and seem experimentally promising. Here, the combined effect of strong spin-orbit coupling, (proximity-induced) superconductivity, and exchange interactions or Zeeman splitting 26 induces MBS located at the opposite ends of a topological phase region. Experiments have so far focused on probing transport signatures of MBS [27-29], such as a zero-bias conductance peak [30-33, but they could not conclusively rule out other topologically trivial origins. (a)

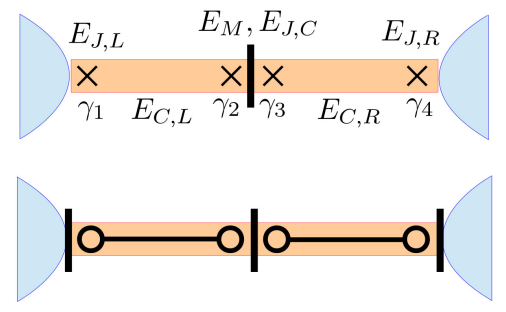

FIG. 1: Segmented nanowire setup for gate-controlled fusion of MBS. The device consists of two tunnel-coupled superconducting islands (orange), formed by a nanowire in proximity to a superconductor (not shown). In addition to that, both islands are connected to nontopological bulk superconductors (blue) with a tunable coupling. In (a), when the outer valves are maximally open (largest coupling) and the central valve is closed, the device hosts four MBS at zero energy (crosses). In (b), when all junctions are closed (minimal coupling), the pairs of MBS on each island are fused (connected circles). In this situation, the device may still possess a subgap state provided $E_{C, \alpha}<\Delta(\alpha=L, R)$, rendering the sketched setup distinct from a conventional nontopological Cooper pair box.

Clear evidence in favor of the topological nature of MBS would instead be provided by verifying their distinctive exchange characteristics. Various approaches to braiding have been suggested, which fall into two categories: One way is to move topological phase boundaries, for example, in nanowire-based proposals through keyboard gates [34 37] or supercurrents [38. Another way is to slowly change the couplings between the MBS to adiabatically manipulate the ground state [39. This can be achieved, 
for example, by magnetic-field control for magnetic atom rings [40] or by tuning electric gates 41] as well as magnetic fluxes 42 in nanowire devices. With the ongoing progress in nanowire fabrication [43 45], especially the fabrication of branched structures [46, 47] essential for braiding, experiments have begun to move forward in this direction.

The successful implementation of braiding necessitates, however, also initialization and readout of MBS and this requires lifting the ground-state degeneracy a process called fusion of MBS [14]. MBS obey nontrivial fusion rules that, in fact, imply braiding 9 and are therefore an interesting subject in itself. Avoiding errors by tuning between the fused and degenerate regime will practically limit the operation speed of topological devices in experiments. The limitations arising from these steps may even be more restrictive than those for manipulating MBS during braiding as we specifically show for nanowire setups. Estimating such time scales, inferred from studying the behavior of the energy spectrum, is thus of the utmost importance to devise future experiments.

A viable strategy to fuse MBS controllably in nanowire setups is to form mesoscopic superconducting islands (see Fig. 1), which form the basis of various nontopological qubits 48, 49, including Cooper pair boxes and transmon qubits [50, 51]. In the topological case, the charging energy $E_{C}$ of the islands introduces an energy splitting $\varepsilon_{P} \sim E_{C}$ between states of different fermion parity. This fuses the MBS as indicated in Fig. 1(b) by connected circles. By coupling the island through a junction to a bulk superconductor, the parity splitting $\varepsilon_{P} \sim\left(E_{J}^{3} E_{C}\right)^{1 / 4} e^{-\sqrt{8 E_{J} / E_{C}}}$ can be made exponentially small by tuning the Josephson energy $E_{J}$ of this junction 52. This introduces MBS at zero energy, at least up to exponential accuracy as indicated in Fig. 1(a) by crosses. The first proposals of this kind envisaged the Josephson couplings to be controlled by magnetic fluxes [42, 53, and parity readout to be accomplished by a cavity, analogous to transmon qubits [54].

A complementary, all-electrical proposal was put forward in Ref. [55]. In contrast to superconducting qubits typically implemented in metallic systems [48, this approach is based on gateable semiconductor nanowire Josephson junctions. Such junctions have been demonstrated experimentally recently for nontopological devices [56, 57] with prospects also for nontopological quantum computation [58. This approach allows the application of experimental tools from quantum-dot experiments, including parity readout by charge sensing and charge pumping. Based on this, a sequence of stepping stones interpolating between MBS detection and quantum computing was suggested, among these the detection of MBS fusion rules and braiding [55. While braiding requires branched nanowire structures, a fusion-rule test could already be realized with a single nanowire: A prototypical device would consist of a superconducting nanowire hosting two islands in series, which are each coupled to a bulk superconductor as sketched in Fig. 1 .

In this paper, we numerically compute the low-energy spectrum for this setup, extending prior studies on coupled Cooper-pair boxes [58] by accounting for both the parity degree of freedom and the charge state of the islands. This goes beyond several other studies of MBS devices for braiding that either exclude charging energy [34 37, 41], or treat charging only effectively [42, 53].

Accounting for charging effects in the entire range between $E_{C} \ll E_{J}$ and $E_{C}>E_{J}$ is necessary to study the crossover between the degenerate and the fused regime both used in the proposal of Ref. [55]. In the latter case, the charge stored in the superconducting Cooper pair condensate cannot be disregarded any more.

In our study, we will identify four different operating regimes for the coupled topological superconducting islands: (i) a charging-dominated regime [see Fig. 1(b)], (ii) a single-island regime, (iii) a double-island regime, and (iv) a regime of four MBS [see Fig. 11(a)]. These regimes can be divided into several subregimes depending on the "fine structure" of the energy spectrum. Mapping out these regimes is useful for the experimental characterization and needed to study the time scales for operating this device.

We further derive conservative time-scale conditions for the fusion-rule testing protocol suggested in Ref. [55]. In this protocol, one changes the Majorana couplings $\left(E_{M}\right)$ and the Josephson couplings $\left(E_{J, L / R}\right)$, which are indicated in Fig. 1(a), in order to tune the system through the above-mentioned regimes (i) $\rightarrow$ (iv). The manipulations have to be made on a time scale $\Delta t$ that is slow in the sense that

$$
\Delta t \gg \frac{\ln \left[\max \left(E_{J, L / R}^{\max }, E_{M}^{\max }\right) / E_{C}\right]}{E_{C}},
$$

but at the same time fast in the sense that

$$
\Delta t \ll \frac{1}{\max \left(\varepsilon_{P}^{\min }, E_{M}^{\min }\right)} .
$$

(We set $\hbar=e=k_{B}=1$.) The above conditions depend on the minimal and maximal values of the couplings, which have to satisfy the condition

$$
E_{C} \ll E_{J}^{\max } \ll\left(E_{M}^{\max }\right)^{2} / E_{C} \ll \Delta^{2} / E_{C} .
$$

Moreover, Eqs. (2) and (3) incorporate the superconducting gap $\Delta$ in the nanowires and the bulk superconductors. The first criterion (1) guarantees that the evolution proceeds adiabatically, that is, transitions from the ground-state manifold into any of the excited state are suppressed. However, there is also a diabaticity condition because the ground-state degeneracy is changed during the protocol. This degeneracy is not perfect in practice (as for any realistic topological device) but a small energy splitting $\varepsilon_{P}^{\min }$ remains. Proceeding adiabatically with respect to this (unwanted) remaining splitting could take the system to the lowest of all energy eigenstates. This has to be avoided by proceeding fast enough so that the 
system state has no time to evolve within the groundstate manifold, which leads to the criterion $\Delta t \ll 1 / \varepsilon_{P}^{\min }$ in Eq. 22. The second condition in Eq. (2) is needed to avoid relaxation processes (tunneling of single electrons between the islands) when they are unwanted. By contrast, resetting the system by going back from (iv) to its initial state at (i) involves a charge-relaxation processes, which contributes to the period of a full cycle [not accounted for by Eq. [2]].

With these insights from the single-wire geometry, we additionally analyze basic operations on MBS in nanowire networks essential for braiding. We show that similar time-scale conditions as Eqs. (1) and (2) have to be satisfied. In the context of braiding, diabatic corrections due to a finite operation time have been discussed in the literature 59, mostly when the MBS are braided through moving domain walls 60 62, but also through changing their couplings [63, 64]. The latter works also suggest error correction procedures based on introducing counterdiabatic correction terms in the Hamiltonian 63, or smoother parameter changes as well as intermediate measurements 64. The focus of our work is on the time scales that are necessary to suppress transition rates into excited states from the start.

Our study does not include the effect of quasiparticles which can lead to parity flips detrimental to the operation of the devices. Thus, $\Delta t$ should also be much smaller than quasiparticle poising times and at least for closed islands this seems to satisfied in view of recent experimental results 65. Our results thus apply when the low-energy spectrum lies below the superconducting gap $\Delta$, which is ensured by the last inequality in Eq. (3). In particular, the charging energies $E_{C, \alpha}$ has to be smaller than the superconducting gap $\Delta$. Moreover, $E_{C, \alpha}$ has to be much larger than temperature since thermal fluctuations of the charge states have to be suppressed for initialization and readout.

The paper is structured as follows: In Sec. III, we introduce our model for the double-island setup shown in Fig. 1. After briefly discussing our numerical approach in Sec. III we analyze the numerically computed energy spectrum in Sec. IV. We find different operating regimes, which can be understood in simple limits from analytic approximations, closely resembling the behavior of coupled oscillators. Based on the energy spectrum, we discuss the time-scale conditions for the fusion-rule testing protocol in Sec. V and for basic manipulations in branched nanowire structures in Sec. VI. Finally, Sec. VII concludes and summarizes our findings. Our analysis is complemented by several appendices, in which we discuss among other things our parameter choices (App. B, extend our analysis of the parameter regimes mentioned above, e.g., for asymmetric setups (App. D), and analyze the time scales for the readout (App. E).

\section{MODEL: GATE-TUNABLE COUPLED SUPERCONDUCTING MAJORANA ISLANDS}

The setup we investigate in this work is sketched in Fig. 1. It consists of a segmented superconducting nanowire, which is at both ends coupled to a bulk superconductor. The superconductivity in the nanowire is proximity induced, for example, by metal deposition [30] or an epitaxially grown shell coating the nanowire [44, 45]. The combined proximal superconductor and the nanowire form what we refer to as superconducting islands (red in Fig. 1). The coupling of these islands to the bulk superconductors and the coupling between the two islands can be controlled electrically by nearby gates. The junctions can thus be operated as valves, which are open for maximal coupling and closed for minimal coupling. When the nanowire is driven into a topologically nontrivial regime, as already mentioned in Sec. II, the islands possess an additional ground-state degeneracy associated with the MBS (see Sec. II B).

We note that the analysis in this paper is not necessarily tied to the specific physical realization mentioned above. One might, for example, also control the Josephson energies by magnetic fluxes [42. However, it is important that all the couplings can be tuned over a wide range from $E_{J} \lesssim E_{C}$ to $E_{J} \gg E_{C}$. To achieve large ratios $E_{J} / E_{C}$ with gate control but without perturbing the MBS, it may be experimentally useful to deviate from the geometry shown in Fig. 1 and instead form a contact from the middle of the nanowire to the ground via a second nanowire allowing gate control of the Josephson energy.

Our goal is to investigate the energy spectrum of this device at energies below $\Delta=\min \left(\Delta_{\text {island }}, \Delta_{\text {bulk }}\right)$, the minimum of the superconducting gaps on the island and in the bulk. We model the device by the following Hamiltonian,

$$
H=\sum_{\alpha=L, R} H_{\alpha}+H_{T}
$$

which consists of three parts: The first two parts, $H_{L}$ and $H_{R}$, describe the two individual superconducting islands and their coupling to the bulk superconductors, and the third part, $H_{T}$, accounts for the tunnel coupling between the islands when opening the central junction. We neglect here capacitive coupling between the islands since there are indications that they are much smaller than the local charging energies 55]. We have verified that small cross-capacitive couplings (much smaller than the local charging energies) do not impair our results for the operating regimes and the time scales discussed below. The reason is that capacitive couplings are relevant only when the charging energies are the dominating energy scale; and otherwise they introduce only minor corrections for the energy spectrum. 


\section{A. Hamiltonian for superconducting islands}

The superconducting islands $(\alpha=L, R)$ are modeled in the standard way for a Cooper pair box [51]:

$$
H_{\alpha}=H_{C, \alpha}+H_{J, \alpha} .
$$

The first term incorporates the classical Coulomb interaction between the electrons on the islands,

$$
H_{C, \alpha}=E_{C, \alpha}\left(\hat{n}_{\alpha}-n_{g, \alpha}\right)^{2},
$$

while the second term accounts for the Josephson couplings to the bulk superconductors:

$$
H_{J, \alpha}=E_{J, \alpha}\left(1-\cos \left(\hat{\varphi}_{\alpha}\right)\right) .
$$

Here, the operator $\hat{n}_{\alpha}$ counts the number of excess electrons on island $\alpha=L, R$ relative to an arbitrary offset. The electron number with the minimal energy can be tuned by nearby gates changing $n_{g, \alpha}$. The operator $\hat{n}_{\alpha}$ is conjugate to the operator $\hat{\varphi}_{\alpha}$ of the phase difference between island and bulk:

$$
\left[\hat{\varphi}_{\alpha}, \hat{n}_{\beta}\right]=2 i \delta_{\alpha \beta}
$$

This means that the phase operator generates changes of the charge 66],

$$
e^{ \pm i \hat{\varphi}_{\alpha}}\left|n_{\alpha}\right\rangle=\left|n_{\alpha} \pm 2\right\rangle
$$

where $\left\{\left|n_{\alpha}\right\rangle: n_{\alpha} \in \mathbb{Z}\right\}$ denotes the orthonormal number basis consisting of states with $n_{\alpha}$ electrons on island $\alpha=$ $L, R$.

One may question at this point whether Eq. 77 is an appropriate model for the Josephson energy for semiconductor nanowire junctions. Recent experiments on gatemons [56, 57 indicate that such junctions connect the island with the bulk through a few, say $N$, channels with large transmission probability $T_{i} \sim 1$ when the valve is opened. Then a different expression for the Josephson Hamiltonian involving higher harmonics in $\hat{\varphi}_{\alpha}$ should be used as discussed in Refs. [55, 56]. Moreover, the charging energy might be renormalized when the transmission amplitudes are not small, similar to Refs. 67, 68. Our model is thus strictly valid only if the transmission probabilities of all channels are small. This implies that we can study the regime $E_{J, \alpha} \gg E_{C, \alpha}$ only under the assumption $E_{C, \alpha} \ll \Delta$ because the Josephson couplings scale as $E_{J, \alpha} \propto \sum_{i=1}^{N} T_{i} \Delta$ 69]. However, we emphasize that all the above-mentioned effects will serve to enlarge the window for the time scales compared with our derivation, which therefore remains a useful conservative estimate.

\section{B. Majorana bound states and basis}

At first sight, Eq. 5 does not seem to differ from a standard Cooper pair box for a topologically trivial superconducting island. What is different here, however, is that the fermion number can be both even and odd, i.e., we have to account for both fermion parity sectors. Introducing the fermion-parity operator,

$$
P=\frac{1}{2}\left(1-(-1)^{\hat{n}_{\alpha}}\right),
$$

one can project Eq. (5) onto the two subspaces according to its eigenvalues $p=0$ (even fermion parity) and $p=1$ (odd fermion parity), respectively, which yields

$$
H_{\alpha}=H_{\alpha}^{p=0}+H_{\alpha}^{p=1} .
$$

There are no off-diagonal blocks between even and odd parity because the Hamiltonians $H_{\alpha}(\alpha=L, R)$ conserve the fermion parity for each island [88]:

$$
\left[H_{\alpha}, P\right]=0 \text {. }
$$

In a topologically trivial superconductor one would account only for the even parity part, $H_{\alpha}^{p=0}$, and omit the odd parity part, $H_{\alpha}^{p=1}$. The reason is that an odd parity state requires an additional quasiparticle mode to be occupied, which is associated with an energy at least as large as the superconducting gap $\Delta$, which is outside the energy regime we are interested in here.

Majorana bound states. The topologically nontrivial superconducting islands each possess an additional fermionic mode, associated with the field operators $\hat{f}_{12}=$ $\left(\hat{\gamma}_{1}+i \hat{\gamma}_{2}\right) / 2$ for the left island and $\hat{f}_{34}=\left(\hat{\gamma}_{3}+i \hat{\gamma}_{4}\right) / 2$ for the right island, respectively. When the outer valves in our device are opened and the central valve is closed, these additional modes derive from pairs of Majorana bound states (MBS) at zero energy, $\gamma_{n}(n=1, \ldots, 4)$, localized at the opposite ends of the wire segments [3, 4] as sketched in Fig. 1(a). The associated self-conjugate Majorana operators, $\hat{\gamma}_{n}=\hat{\gamma}_{n}^{\dagger}$, are denoted with hats and satisfy anticommutation relations $\left\{\hat{\gamma}_{n}, \hat{\gamma}_{m}\right\}=2 \delta_{n m}$. We assume that the two Majorana wave functions on each island do not overlap in space. As a consequence, occupying the fermionic modes $f_{12}$ and $f_{34}$ is associated with zero "orbital" energy. Hence, both fermion parity sectors are accessible at low energies $<\Delta$, in contrast to a topologically trivial superconductor. The degeneracy between the even and odd parity sectors can be lifted either by the charging energy [see Fig. 11(b)] [66] or by the tunnel coupling of the central valve, which in both cases fuses MBS.

Phase basis and Majorana operators. We give an intuitive definition of the Majorana operators first in the phase basis $\left|\varphi_{L}, \varphi_{R}\right\rangle$, which is the Fourier transform of the number basis. First considering only the left island, we define (see App. A 66

$$
\begin{aligned}
\left|0_{12}, \varphi_{L}\right\rangle & =\frac{1}{\sqrt{2 \pi}} \sum_{n_{L} \text { even }} e^{-i \varphi_{L} n_{L} / 2}\left|n_{L}\right\rangle, \\
\left|1_{12}, \varphi_{L}\right\rangle & =\frac{1}{\sqrt{2 \pi}} \sum_{n_{L} \text { odd }} e^{-i \varphi_{L} n_{L} / 2}\left|n_{L}\right\rangle,
\end{aligned}
$$

where the variable $\varphi_{L} \in[0,2 \pi)$ is continuous and $p_{12}=0_{12}, 1_{12}$ denotes the occupation of fermionic mode 
$f_{12}=\gamma_{1}+i \gamma_{2}$ and thus the fermion parity of the island [89]. When used as a label, we add a subscript (here 12) to the numbers 0 and 1 to denote the fermionic mode that is meant; however, in mathematical expressions $p_{12}$ should be evaluated as the numbers 0 and 1 . The wave functions can be represented in phase space as $\psi_{p_{12}}\left(\varphi_{L}\right)=\left\langle p_{12}, \varphi_{L} \mid \psi\right\rangle$, which obey the periodicity condition

$$
\psi_{p_{12}}\left(\varphi_{L}+2 \pi\right)=(-1)^{p_{12}} \psi_{p_{12}}\left(\varphi_{L}\right)
$$

The action of the Majorana operators $\hat{\gamma}_{1}$ and $\hat{\gamma}_{2}$ on the wave functions can then be defined through

$$
\begin{aligned}
& e^{ \pm i \hat{\varphi}_{L} / 2} \hat{\gamma}_{1} \psi_{p_{12}}\left(\varphi_{L}\right)=e^{ \pm i \varphi_{L} / 2} \psi_{\bar{p}_{12}}\left(\varphi_{L}\right) \\
& e^{ \pm i \hat{\varphi}_{L} / 2} \hat{\gamma}_{2} \psi_{p_{12}}\left(\varphi_{L}\right)=e^{ \pm i \varphi_{L} / 2} i^{1-2 p_{12}} \psi_{\bar{p}_{12}}\left(\varphi_{L}\right)
\end{aligned}
$$

where $\bar{p}_{12}=1-p_{12}$. We added the phase factors here because the Majorana operators appear only in combination with them in the tunneling Hamiltonian 20 discussed below (which is all we need). Moreover, because of the phase factors $e^{ \pm i \varphi_{L} / 2}$, the phase-space wave functions on the right-hand side of Eqs. (16) and (17) obey automatically the boundary conditions (15). We give a derivation of the above relations in App. A1 starting from a standard BCS description of the island.

Since we express the Hamiltonian in the number basis for our numerics, we further note the following useful relations:

$$
\begin{aligned}
e^{ \pm i \hat{\varphi}_{L} / 2} \hat{\gamma}_{1}\left|n_{L}\right\rangle & =e^{ \pm i \hat{\varphi}_{L} / 2} i^{2 n_{L}-1} \hat{\gamma}_{2}\left|n_{L}\right\rangle \\
& =\left|n_{L} \pm 1\right\rangle .
\end{aligned}
$$

Corresponding relations hold when replacing $L \rightarrow R$ and $12 \rightarrow 34$ for the right island and a full basis can be formed by tensor-product states.

\section{Majorana-Josephson coupling}

Without tunnel coupling across the center junction, the even and odd parity sectors for each of the islands decouple. This changes with a tunnel coupling, which we model with the following Hamiltonian:

$$
\begin{aligned}
H_{T}= & E_{J, C}\left(1-\cos \left(\hat{\varphi}_{L}-\hat{\varphi}_{R}\right)\right) \\
& +E_{M} \cos \left(\frac{\hat{\varphi}_{L}-\hat{\varphi}_{R}}{2}\right) i \hat{\gamma}_{2} \hat{\gamma}_{3} .
\end{aligned}
$$

The first term is the "conventional" Cooper pair tunneling associated with a Josephson energy $E_{J, C}$, which conserves the fermion parities of both islands. The second term, also known as the fractional Josephson effect [20], involves parity flips. We derive the Majorana-Josephson term in App. A2, which also shows that the combination $i \hat{\gamma}_{2} \hat{\gamma}_{3}$ appears naturally since the tunnel coupling is local. The Majorana-Josephson term can be interpreted most clearly by comparing with its representation in the number basis [using Eq. [19]]:

$$
H_{M}=-\frac{E_{M}}{2} \sum_{n_{\alpha}, \eta= \pm 1}\left|n_{L}-\eta, n_{R}+\eta\right\rangle\left\langle n_{L} n_{R}\right| .
$$

Equations (20) and (21) together show that the transfer of single electrons across the central junction [described by Eq. [21]] leads to a transfer of charge between the islands [through the phase-dependent terms in Eq. 201] as well as a flip of their fermion parities [through the Majorana operators in Eq. 200].

We note that in combining Eq. (20) with the island Hamiltonians (5) in the full Hamiltonian (4), we assume that there is no phase difference across the two bulk superconductors. This is further discussed in App. B1 and motivated mainly by the fact that any phase difference would increase the ground-state energy (and for the protocols we discuss the system should stay mostly in the ground state, at least when phase differences could be relevant).

Even though $E_{J, C}$ and $E_{M}$ appear as independent parameters in Eq. (4), they cannot be controlled individually in an experiment with a gate at the central junction. We estimate in App. B2 that they are related by $E_{J, C} \sim E_{M}^{2} / \Delta$ for typical parameters. The Josephson energy $E_{J, C}$ and the Majorana coupling $E_{M}$ may therefore become of comparable size only when $E_{M}$ approaches the superconducting gap $\Delta$. Since we assume $E_{M} \ll \Delta$ during MBS manipulations, we will thus set $E_{J, C}=0$ in some parts of our analysis, which simplifies the considerations. However, we point out that a nonzero central Josephson coupling is not detrimental to the gate-controlled approach for manipulating MBS.

Total parity conservation. The Hamiltonian (4) conserves the total fermion parity:

$$
[H, P]=0 .
$$

This is seen from the representation 21 of the Hamiltonian in the number basis. Only the fermion parity of the individual islands may be changed by the tunneling process. In contrast to the total charge, the total parity is thus always a good quantum number 90]. The coupling to the environment can break fermion parity conservation (so-called quasiparticle poisoning), which is an experimentally relevant issue. Including such processes is beyond the scope of this paper; a brief discussion of this issue can be found in Ref. [55]. Basically, we expect that such processes happen on time scales long compared to those on which MBS will be operated in such devices.

\section{Hamiltonian in sum and difference variables}

The above terms of the Hamiltonian are expressed in the phase and number operators referring to the individual islands. For our physical discussion of the energy 
spectra, it will be useful to express the Hamiltonian instead in the sums and differences of the phase and number operators,

$$
\begin{aligned}
& \left(\begin{array}{c}
\hat{\Phi} \\
\Delta \hat{\varphi}
\end{array}\right)=\left(\begin{array}{cc}
1 & 1 \\
1 & -1
\end{array}\right)\left(\begin{array}{l}
\hat{\varphi}_{L} \\
\hat{\varphi}_{R}
\end{array}\right), \\
& \left(\begin{array}{c}
\hat{N} \\
\Delta \hat{n}
\end{array}\right)=\left(\begin{array}{cc}
1 & 1 \\
1 & -1
\end{array}\right)\left(\begin{array}{l}
\hat{n}_{L} \\
\hat{n}_{R}
\end{array}\right),
\end{aligned}
$$

which is merely a canonical transformation (up to normalization constants). These operators form again a set of canonically conjugate operator pairs:

$$
\begin{aligned}
{[\hat{\Phi} / 2, \hat{N} / 2] } & =[\Delta \hat{\varphi} / 2, \Delta \hat{n} / 2]=i, \\
{[\hat{\Phi}, \Delta \hat{n}] } & =[\hat{N}, \Delta \hat{\varphi}]=0 .
\end{aligned}
$$

In terms of these operators, the Hamiltonian reads

$$
\begin{aligned}
H= & 2 \bar{E}_{C}\left[\left(1-\varepsilon_{C}^{2}\right)\left(\frac{\hat{N}-N_{g}}{2}\right)^{2}+\left(\frac{\Delta \hat{n}-\Delta n_{g}}{2}+\varepsilon_{C} \frac{\hat{N}-N_{g}}{2}\right)^{2}\right]+2 \bar{E}_{J}\left[1-\cos \left(\frac{\hat{\phi}}{2}\right) \cos \left(\frac{\Delta \hat{\varphi}}{2}\right)-\varepsilon_{J} \sin \left(\frac{\hat{\phi}}{2}\right) \sin \left(\frac{\Delta \hat{\varphi}}{2}\right)\right] \\
& +E_{M} \cos \left(\frac{\Delta \hat{\varphi}}{2}\right) i \hat{\gamma}_{2} \hat{\gamma}_{3}+E_{J, C}(1-\cos (\Delta \hat{\varphi}))
\end{aligned}
$$

This contains the averages,

$$
\bar{E}_{C}=\frac{E_{C, L}+E_{C, R}}{2}, \quad \bar{E}_{J}=\frac{E_{J, L}+E_{J, R}}{2},
$$

and asymmetry parameters,

$$
\varepsilon_{C}=\frac{E_{C, L}-E_{C, R}}{E_{C, L}+E_{C, R}}, \quad \varepsilon_{J}=\frac{E_{J, L}-E_{J, R}}{E_{C, L}+E_{C, R}},
$$

as well as the sum and difference of the gatings:

$$
\begin{aligned}
N_{g} & =n_{g, L}+n_{g, R}, \\
\Delta n_{g} & =n_{g, L}-n_{g, R} .
\end{aligned}
$$

This completes our discussion of the model Hamiltonian and we explain next how we diagonalize it numerically.

\section{NUMERICAL DIAGONALIZATION}

We compute the spectrum of the model Hamiltonian (4) by expressing it as a matrix in the number basis $\left|n_{L}, n_{R}\right\rangle=\left|n_{L}\right\rangle \otimes\left|n_{R}\right\rangle$ based on Eqs. (6), (7), and (20) together with Eqs. (9) and (21). Since the Hamiltonian (4) conserves the total parity, both the total parity sectors can be diagonalized individually for all parameters. We restrict our calculations to the subspace of even total parity unless stated otherwise.

For the numerical diagonalization, we introduce a cutoff for the maximal electron number that we include: $\left|n_{L}\right|,\left|n_{R}\right| \leqslant N_{\max }$. This is sufficient because the charging energy acts like a parabolic potential for a "particle" that is confined in number space. In this analogy, the kinetic energy of the particle is given by the energy scale $E_{T}=\max \left(E_{J, C}, E_{M}, E_{J, L}, E_{J, R}\right)$. If the potential energy $\sim E_{C}\left(n_{L}^{2}+n_{R}^{2}\right)$ exceeds the kineticenergy scale $E_{T}$, the contributions from the corresponding number states become exponentially small for the lowest eigenstates. Thus, one may neglect states for $n_{L}, n_{R} \gtrsim N_{\max }=\sqrt{E_{T} / E_{C}}$. Since we keep $E_{T} \lesssim 100 E_{C}$ in our simulations, choosing a cutoff $N_{\max }>10$ already yields the low-energy spectrum with high accuracy.

Diagonalizing the Hamiltonian in the number basis turns out to be much more convenient in the presence of charging energies as compared to diagonalizing it in the phase basis as we further substantiate in App. C.

\section{LOW-ENERGY SPECTRUM}

We next discuss the low-energy spectrum of the coupled topological superconducting islands. In addition to providing an overview of the different operating regimes, this is also of experimental interest: A thorough characterization of such devices will be needed as a preparation step before the protocols presented in Ref. [55] can be implemented experimentally.

To keep the analysis simple in this Section, we neglect the Josephson energy of the central junction $\left(E_{J, C}=0\right)$ and assume symmetric islands $\left(E_{J, L}=E_{J, R}=E_{J}\right.$ and $\left.E_{C, L}=E_{C, R}=E_{C}\right)$. We dedicate App. D to investigating effects of deviating from these assumptions. Analyzing the spectrum under the simplifying assumptions has the advantage that it provides a conservative estimate of the time scales for operating MBS in these devices (see Sec. VP.

The characteristics of the spectrum can be divided into four different parameter regimes as illustrated in Fig. 2 (i) If the charging energy dominates all other energy scales, $E_{C} \gg E_{J}, E_{M}$, the system behaves as two closed, uncoupled islands (green in Fig. 2, see Sec. IV A). The islands host a definite number of electrons except for charge-degeneracy points, depending on the gatings $n_{g, L}$ and $n_{g, R}$. (ii) If the Majorana tunneling dominates, 
$E_{M} \gg E_{J}, E_{C}$, the system behaves as a single, larger island at low energies $<\sqrt{4 E_{M} E_{C}}$ (orange in Fig. 2 , see Sec. IVB). The superconducting phases of both islands are then locked to each other. At larger energies $>\sqrt{4 E_{M} E_{C}}$, the dynamics of their phase difference has to be taken into account.

(iii) If instead the Josephson coupling dominates, $E_{J} \gg E_{M}, E_{C}$, the system has to be treated rather as two separate open islands (blue in Fig. 2, see Sec. IV C). Here, the phases of the islands have to be treated as individual degrees of freedom even at low energies. (iv) Finally, there is the four-MBS regime (yellow in Fig. 2, see Sec. IV D, which appears for two open uncoupled islands. Here, both the tunnel coupling of the MBS across the center junction and the charging-mediated couplings of the MBS on each island are strongly suppressed.

Representative plots of the energy spectra along paths in the $\left(E_{J}, E_{M}\right)$ plane shown in Fig. 2 are shown in Fig. 3. The color of the horizontal axes in Fig. 3 corresponds to the regimes shown in Fig. 2 From Fig. 3 it is clear that the dashed lines in Fig. 2, marking the boundaries of the different regimes, should not be understood as lines of a "phase transition". The transition from one regime to the other is gradual and may even be shifted for higher-lying excited states. Figure 2 should thus be understood rather as a rough guide for the characteristics of the spectra. We next discuss the different regimes in detail.

\section{A. Two closed uncoupled islands: $E_{C} \gg E_{M}, E_{J}$}

When the charging energy $E_{C}$ dominates, the eigenstates are close to the number states $\left|n_{L}, n_{R}\right\rangle$ except at degeneracy points where $n_{g, \alpha}= \pm 1 / 2, \pm 1, \pm 3 / 2, \ldots$. This results in a charge-stability diagram similar to nonsuperconducting double-dot devices [70] but with the constraint that the total parity is conserved (parityswitching processes are not considered here). The eigenenergies are roughly given by $E=E_{C, L} n_{L}^{2}+$ $E_{C, R} n_{R}^{2}$, which approximately reproduces the low-energy spectra shown in the left (green) parts of Figs. 3(a) and (d) for $E_{M}, E_{J} \ll E_{C}$.

The MBS $\left(\gamma_{1}, \gamma_{2}\right)$ and $\left(\gamma_{3}, \gamma_{4}\right)$ are fused in this regime, which is a viable way to initialize and readout MBS (see Sec. Vp. Away from degeneracy points, the eigenstates of different fermion parity possess a different charge parity and charge are not independent.

\section{B. Single island: $E_{C}, E_{J} \ll E_{M}$}

When the Majorana coupling between the islands is the largest energy scale, the physics can be understood most clearly from the representation 27) of the Hamiltonian in the sum and difference variables. Equation 27) can here be interpreted analogous to a pair of strongly coupled oscillators, identifying the phases with positions and the number of electrons with momentum. For $E_{M} \gg E_{J}, E_{C}$, the dynamics of the "relative coordinate" $\Delta \varphi$ is fast since it is subject to a strong confining potential, while the dynamics of the "center coordinate" $\Phi$ is slow since it is much more weakly confined.

To understand the energy spectrum, we first decompose the Hamiltonian into $H=H_{\Delta}+H_{\Sigma}$ with

$$
\begin{aligned}
& H_{\Delta}=2 E_{C}\left(\frac{\Delta \hat{n}-\Delta n_{g}}{2}\right)^{2}+E_{M} \cos \left(\frac{\Delta \hat{\varphi}}{2}\right) i \hat{\gamma}_{2} \hat{\gamma}_{3}, \\
& H_{\Sigma}=2 E_{C}\left(\frac{\hat{N}-N_{g}}{2}\right)^{2}+2 E_{J}\left[1-\cos \left(\frac{\Delta \hat{\varphi}}{2}\right) \cos \left(\frac{\hat{\Phi}}{2}\right)\right] .
\end{aligned}
$$

On a rough energy scale, the spectrum is determined by $H_{\Delta}$, while $H_{\Sigma}$ can be treated as a perturbation for $E_{M} \gg E_{J}, E_{C}$. First setting $H_{\Sigma}=0$, the Hamiltonian is formally the same as that of a Cooper pair box, with the physical difference that the Majorana term couples consecutive electron number states on the islands instead of consecutive Cooper pair number states [91]. The eigenenergies are approximately given by

$$
\begin{aligned}
E_{k_{\Delta}}= & \sqrt{4 E_{C} E_{M}}\left(k_{\Delta}+1 / 2\right) \\
& -\left(E_{C} / 4\right)\left(k_{\Delta}^{2}+k_{\Delta}+1 / 2\right)+O\left(\sqrt{E_{C} / E_{M}}\right)
\end{aligned}
$$

with $k_{\Delta}=0,1, \ldots$. We obtained this result by expanding $H_{\Delta}$ in $\Delta \varphi$ and including anharmonic corrections perturbatively along the lines of Ref. [51]. The resulting lowestorder energy gaps of $\sqrt{4 E_{C} E_{M}} k_{\Delta}$ to the ground state are indicated as red-dashed lines in Fig. 3(a). We note that the energy levels are altered when the central Josephson energy is included, which we discuss further in App. D 1.

We next discuss the effect of the "fine-structure" term $H_{\Sigma}$, which gives rise to smaller energy splittings than those induced by $H_{\Delta}$. Depending on the bulk Josephson coupling $E_{J}$, we find two subregimes, which are marked in Fig. 2 in two shades of orange.

(i) Closed islands: $E_{J} \ll E_{C}\left(\ll E_{M}\right)$. Let us first consider the simplest case of $E_{J}=0$, the situation shown in the orange part of Fig. 3(a): Here, the total number of electrons on the islands is conserved, $[\hat{N}, H]=0$, and $\hat{N}$ can thus be treated as a number $N$. In Fig. 3(a), we highlight the energy gaps of all states with zero excess charge, $N=0$, labeled by $\left(k_{\Delta}, 0\right)$ in darker color (corresponding to $H_{\Sigma}=0$ ). The full energy spectrum including all other states with (even) $N \neq 0$ is then obtained as replicas of the spectrum for $N=0$ by adding the charging energies $H_{\Sigma}=2 E_{C}(N / 2)^{2}$. The corresponding energy differences to the total ground state are shown in Fig. 3(a) in brighter color. Among those, we see a sequence of constant energy gaps. These correspond to the energetically lowest states $\left(k_{\Delta}=0\right)$ for the different values of $N \neq 0$. Correspondingly shifted "replicas" of 


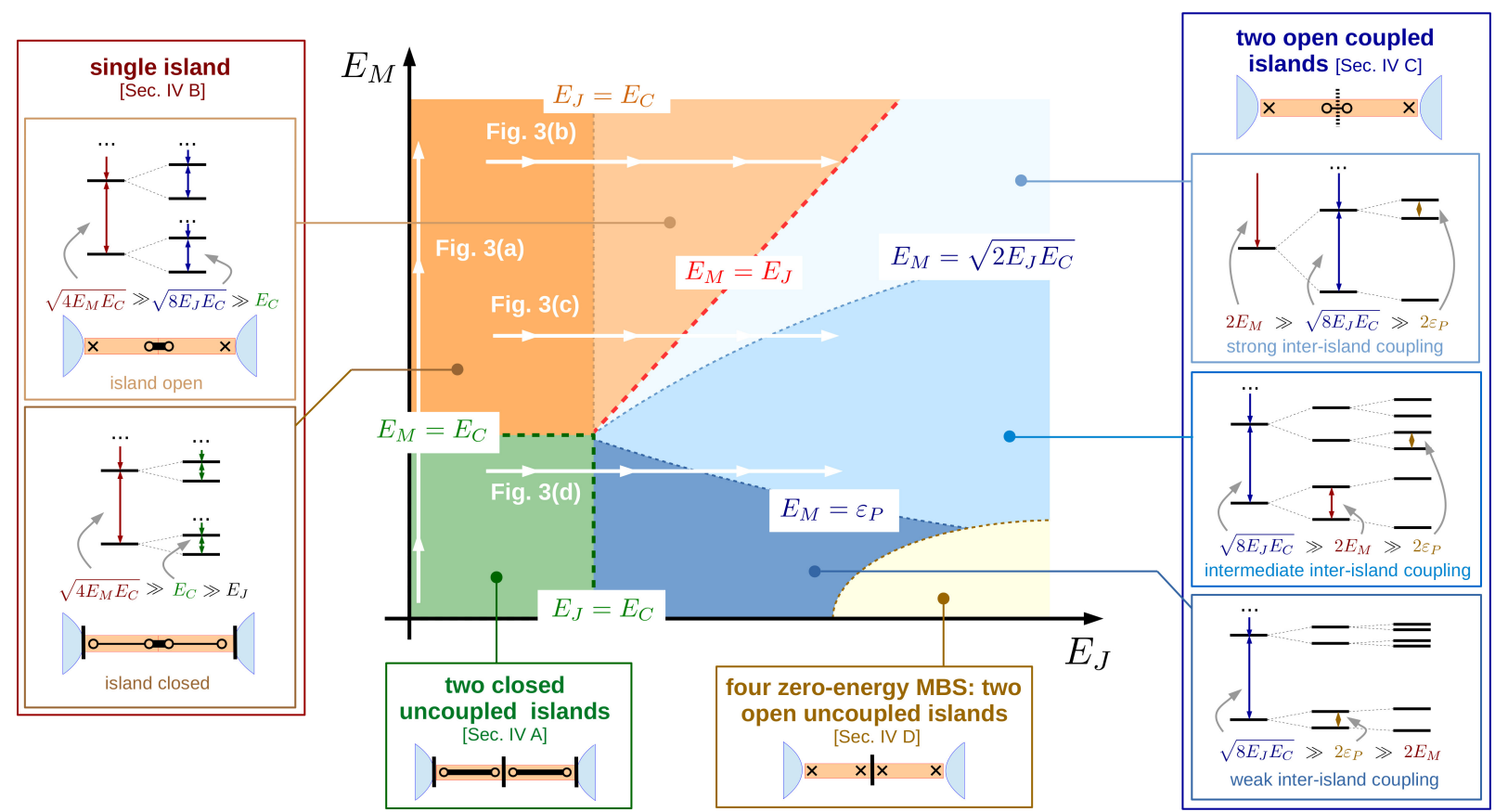

FIG. 2: Sketch of the parameter regimes for the energy spectra characteristics of the coupled topological superconducting island Hamiltonian (4). In the boxes, we sketch the energy level spectrum and indicate the different energy scales dominating the level structure. The lower part of the boxes shows the couplings (lines) between the MBS, which are fused when denoted as circles and at close to zero energy when denoted as crosses. We assume symmetric islands, $E_{J, L}=E_{J, R}=E_{J}, E_{C, L}=E_{C, R}=E_{C}$, and have set the central Josephson coupling to zero, $E_{J, C}=0$. The white arrows indicate the paths taken in the $\left(E_{J}, E_{M}\right)$ plane for the plots in Fig. 3

the states with excited oscillation quanta $\left(k_{\Delta}=1, \ldots\right)$ can also be identified.

(ii) Open islands: $E_{C} \ll E_{J}\left(\ll E_{M}\right)$. Treating the "fine-structure" term (33) as a perturbation, we replace in leading order $\cos (\Delta \varphi / 2)$ by its average:

$$
H_{\Sigma} \approx 2 E_{C}\left(\frac{\hat{N}}{2}\right)^{2}+\frac{E_{J}}{2}(1-\rho)\left(\frac{\hat{\Phi}}{2}\right)^{2}+\rho,
$$

and the eigenenergies are $E=E_{k_{\Delta}}+\Delta E_{k_{\Sigma}}$ with

$$
\Delta E_{k_{\Sigma}}=\sqrt{8 E_{C} E_{J}(1-\rho)}\left(k_{\Sigma}+1 / 2\right)+\rho .
$$

Here, $k_{\Sigma}=0,1, \ldots$ and $\rho=E_{k_{\Delta}} / 2 E_{M}$. Thus, each $k_{\Delta}$ is associated with a ladder of states and each of these ladder states is specified by $k_{\Sigma}$. The different ladders are spaced by the large energy $E_{k_{\Delta}} \approx \sqrt{4 E_{C} E_{M}}$ and the states within each ladder are spaced by an energy $\sim \sqrt{8 E_{J} E_{C}}$. Note that for $E_{J} \gg E_{C}$, anharmonic corrections to $E_{k_{\Delta}}$ can be neglected as compared to the latter splitting.

The crossover from regime (i) to (ii) is indicated by the transition from the dark orange to the light orange part in Fig. 3. (b). If we focus on low energies $E \ll \sqrt{4 E_{C} E_{M}}$, all states correspond to $k_{\Delta}=0$, i.e., there are no excitations corresponding to oscillations in the difference phase $\Delta \varphi$ involved. This physically means that both phases are rigidly coupled at low energies and the two islands behave as one.
In view of the Majorana physics, this means that the "nonlocal" parity $\left\langle i \hat{\gamma}_{2} \hat{\gamma}_{3}\right\rangle=-1$, indicating that the interisland fermionic mode, associated with the annihilator $\hat{f}_{23}=\hat{\gamma}_{2}+i \hat{\gamma}_{3}$, is empty. The MBS pair $\left(\gamma_{2}, \gamma_{3}\right)$ is thus fused - the occupation of $f_{23}$ requires a finite energy $E_{M}$, which is much larger than all other energy scales. For low energies, the fermion parity degree of freedom is gapped out (recall that the spectra are shown here only for even total parity).

\section{Two open islands: $E_{C}, E_{M} \ll E_{J}$}

We next turn to the more intricate case when the Josephson energies of the junctions with the bulk superconductors dominate. Here, $\varphi_{L}$ and $\varphi_{R}$ are not rigidly coupled to each other at low energies (as in the foregoing section) and their individual dynamics becomes important. The low-energy spectrum depends crucially on how the Majorana coupling energy $E_{M}$ compares with (i) the Josephson plasma frequency $\sqrt{8 E_{J} E_{C}}$ and (ii) the charging-induced energy splitting of even and odd fermion parity states of the individual islands. The corresponding energy scale [53] is

$$
\varepsilon_{P}=\frac{32}{\left(2 \pi^{2}\right)^{1 / 4}}\left(E_{J}^{3} E_{C}\right)^{1 / 4} e^{-\sqrt{8 E_{J} / E_{C}}},
$$


(a) $E_{J} / E_{C}=0$

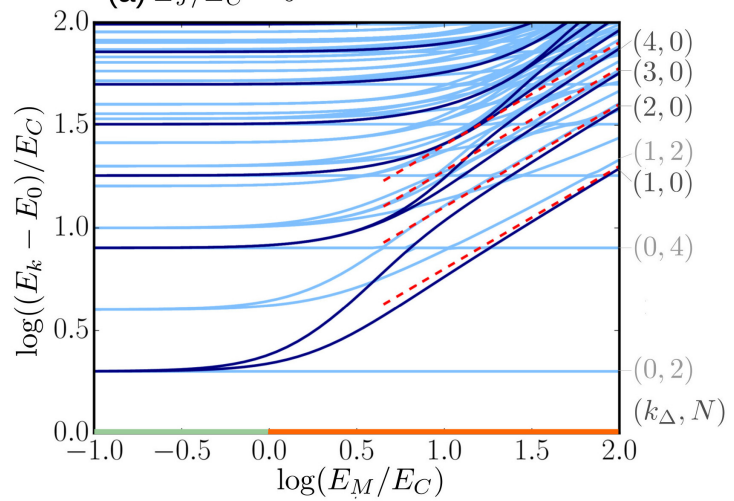

(c) $E_{M} / E_{C}=10^{+1}$

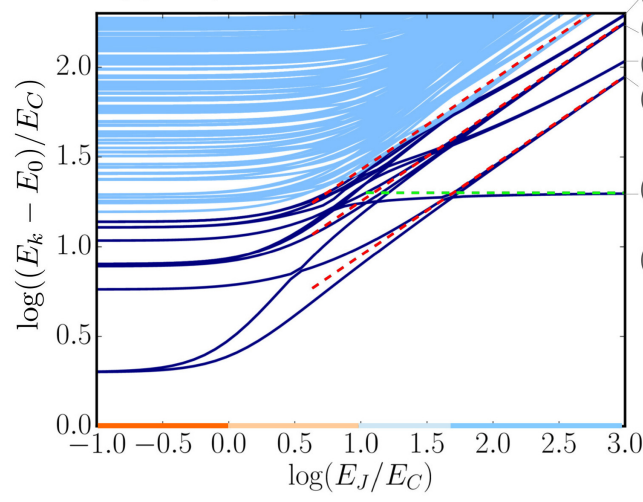

$\left(2,1_{23}\right)$ $\left(2,0_{23}\right)$ $\left(1,1_{23}\right)$ $\left(1,0_{23}\right)$ $\left(0,1_{23}\right)$ $\left(k, p_{23}\right)$ (b) $E_{M} / E_{C}=10^{+3}$
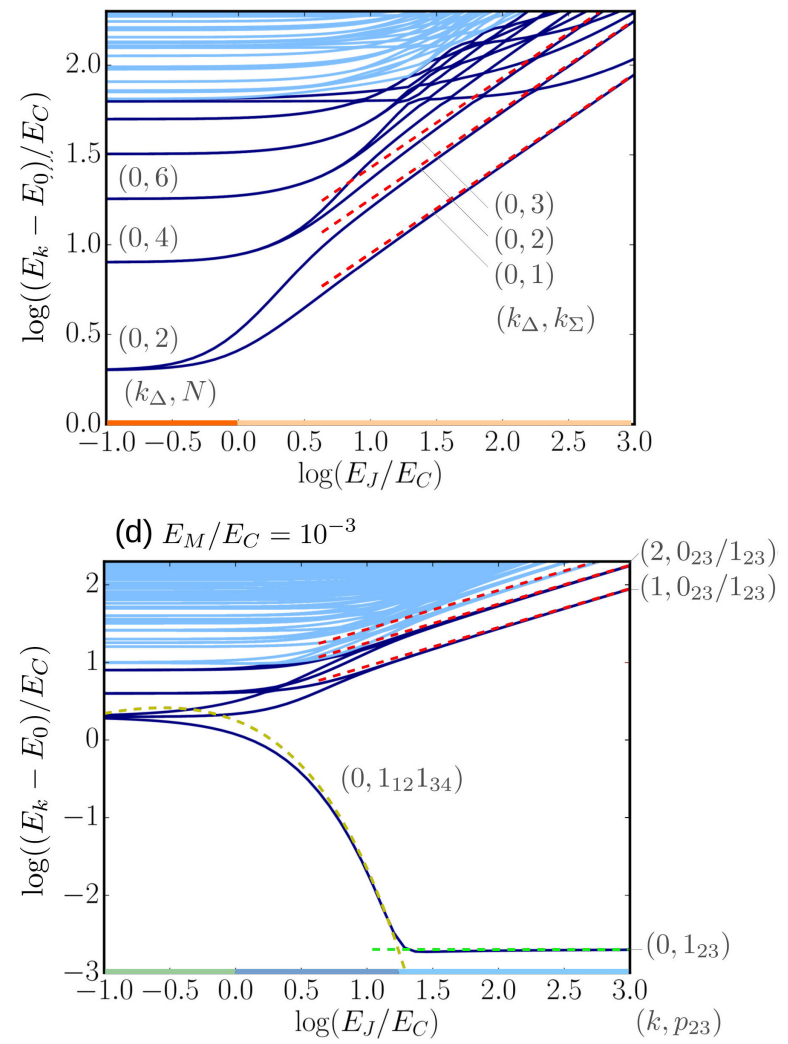

FIG. 3: Low-energy spectrum of coupled topological superconducting islands. Solid lines are the numerically computed energy splittings (blue) between succeeding excited states (energy $E_{k}$ ) and the ground state (energy $E_{0}$ ) in the even total parity sector. Dashed lines indicate analytic approximations to the energy gaps (see below). The parameters are changed along the paths in the $\left(E_{J}, E_{M}\right)$ plane as denoted by the white arrows in Fig. 2 and the colors on the horizontal axis correspond to the regimes in Fig. 2 In (a), we show the spectrum as a function of a the Majorana coupling $E_{M}$ for $E_{J}=0$. We indicate in darker color those states with zero excess charge, $N=0$, while the bright color corresponds to other excess excess charge, $N \neq 0$. The red-dashed lines indicate the analytic approximations from Eq. (35). In (b)-(d), we show the spectra as a function of the bulk Josephson coupling $E_{J}$ for different values of $E_{M}$ as indicated. Here, the darker color highlights the 12 lowest eigenstates and the bright color corresponds to higher-lying states. The dashed lines indicate the transition energies $E_{k}-E_{0}=k \sqrt{8 E_{J} E_{C}}$ related to Josephson plasma oscillations (red), the parity splitting $2 \varepsilon_{P}$, Eq. (37) (yellow), and the Majorana splitting $2 E_{M}$ (green). We assume $E_{C, L}=E_{C, R}=E_{C}, E_{J, L}=E_{J, R}=E_{J, C}=0, n_{g, L}=n_{g, R}=0, E_{J, C}=0$, and use a number-state cutoff $N_{\max }=25$ for the numerical calculations.

which is much smaller than $\sqrt{8 E_{J} E_{C}}$. This yields three possibilities, which are shown in Fig. 2 as three shades of blue and which we discuss next.

(i) Weak inter-island coupling: $2 E_{M} \ll 2 \varepsilon_{P} \ll$ $\sqrt{8 E_{J} E_{C}}$ : In this regime, the system behaves as two weakly coupled topological superconducting islands. To discuss the physics, let us first set $E_{M}=0$ so that $H\left(E_{M}=0\right)=\sum_{\alpha} H_{\alpha}\left(\hat{n}_{\alpha}, \hat{\varphi}_{\alpha}\right)$ simply decomposes into the two island parts. The eigenstates of the system are trivially the product states

$$
\begin{aligned}
& \left|k_{L} k_{R} ; 0_{12} 0_{34}\right\rangle=\left|k_{L} 0_{12}\right\rangle \otimes\left|k_{R} 0_{34}\right\rangle, \\
& \left|k_{L} k_{R} ; 1_{12} 1_{34}\right\rangle=\left|k_{L} 1_{12}\right\rangle \otimes\left|k_{R} 1_{34}\right\rangle .
\end{aligned}
$$

Here, $\left|k_{\alpha}, p_{n m}\right\rangle$ are the excitations of the Josephson plasma oscillations of each island with $k_{\alpha}$ excited quanta and parity $p_{n m}$ of the fermionic mode $\hat{f}_{n m}=\hat{\gamma}_{n}+i \hat{\gamma}_{m}$.
The total energies are approximated by

$$
\begin{aligned}
E \approx & \sqrt{8 E_{J} E_{C}}\left(k_{L}+k_{R}+1\right) \\
& +\left(p_{12}+p_{34}-1\right) \varepsilon_{P},
\end{aligned}
$$

On a rough energy scale, the spectrum can be grouped into pairs of $\left(k_{L}+k_{R}+1\right)$-fold degenerate states, split by the Josephson plasma frequency $\sqrt{8 E_{J} E_{C}}$ (see Fig. 2). The corresponding splittings are denoted by red-dashed lines in the right blue parts of Figs. 3(c) and (d) with $k=k_{L}+k_{R}$. These are valid not only for the weak inter-island coupling, $E_{M} \ll \varepsilon_{P}$, but also for the other subregimes discussed in this section.

Within each of the "parity pairs", the odd-odd parity combination is split from the lower even-even parity combination $\left[92\right.$ by the smaller energy $2 \varepsilon_{P}$ (we focus now on the case $\left.E_{M} \ll \varepsilon_{P}\right)$. For the two lowest lying states with 
$k_{L}=k_{R}=0$, the excited state $\left|00 ; 1_{12} 1_{34}\right\rangle$ is therefore split from the ground state $\left|00 ; 0_{12} 0_{34}\right\rangle$ by $2 \varepsilon_{P}$ as given by Eq. (37), which is indicated by the yellow-dashed line in the dark blue part of Fig. 3(d). We have verified that a nonzero capacitive coupling between the islands, modeled by a term $E_{C, L R} \hat{n}_{L} \hat{n}_{R}\left(E_{C, L R} \leq E_{C}\right)$, influences this parity energy splitting only slightly and does not affect the exponential suppression. We also neglected anharmonic corrections of $O\left(E_{C}\right)$ to the Josephson-plasma frequency in Eq. 40 because these only shift levels of the same $k_{L}+k_{R}$ by the same amount but do not contribute their splitting. Compared to the rough energy scale $\sqrt{8 E_{J} E_{C}}$, these anharmonic corrections are negligible.

When $E_{M} \ll \varepsilon_{P}$, the Majorana tunneling has little effect on the spectrum. For the lowest parity pair, a nonzero $E_{M}$ results in a small shift of the energy levels. This is different for the higher-lying excited states, which exhibit degeneracies that may be lifted for nonzero $E_{M}$ (not resolved on the scale shown in Fig. 3).

(ii) Intermediate inter-island coupling: $2 \varepsilon_{P} \ll$ $2 E_{M} \ll \sqrt{8 E_{J} E_{C}}$. When the Majorana coupling exceeds the charging-induced parity splitting, the interisland tunneling strongly mixes the even-even and oddodd parity sectors. The energy eigenstates are therefore "bonding" and "antibonding" combinations of the "local" parity states:

$$
\begin{aligned}
\left|k_{L} k_{R} ; 0_{23} 0_{14}\right\rangle & =\left|k_{L} k_{R}\right\rangle \otimes \frac{\left|0_{12} 0_{34}\right\rangle+\left|1_{12} 1_{34}\right\rangle}{\sqrt{2}} \\
\left|k_{L} k_{R} ; 1_{23} 1_{14}\right\rangle & =\left|k_{L} k_{R}\right\rangle \otimes \frac{\left|0_{12} 0_{34}\right\rangle-\left|1_{12} 1_{34}\right\rangle}{\sqrt{2}}
\end{aligned}
$$

split by an energy $2 E_{M}$ [green dashed in Figs. 3(c) and (d)]. The crossover from regime (i) to (ii) can be clearly seen for the lowest-lying excited state in Fig. 3(d) from the dark blue to the lighter blue part. As our notation in Eqs. (41) and 42 suggests, increasing the tunnel coupling fuses the MBS in a complementary way: The MBS at the central junction become more strongly fused than the pairs on each island. This is a key ingredient to test the Majorana fusion rules as discussed in Sec. V]

(iii) Strong inter-island coupling: $2 \varepsilon_{P} \ll \sqrt{8 E_{J} E_{C}} \ll$ $2 E_{M}\left(\ll 2 E_{J}\right)$. In this regime, the tunnel coupling between the two islands is not a "fine-structure" effect: Roughly speaking, the lower end of the spectrum is given by bonding states of the two islands, while the upper end of the spectrum is given by antibonding states (see Fig. 2. This effectively removes the parity degree of freedom from the low-energy spectrum, similar to the single-island regime. Accordingly, the Josephson plasmon excitations do not appear in parity pairs.

This can be seen in Fig. 3(c) in the light-blue part: Here, the lowest state of flipped nonlocal parity, labeled by $\left(0,1_{23}\right)$, is at a much higher energy than other states with the same nonlocal parity $\left(k>0,0_{23}\right)$ as the ground state. This contrasts with the situation for the darker blue parts shown in the Fig. 3(c) and in Fig. 3(d), where the state $\left(0,1_{23}\right)$ is closest to the ground state.
Finally, we emphasize that there is still a difference between the regime of strongly coupled individual islands $\left(E_{J} \gg E_{M}, E_{C}\right)$ and the regime of a single island $\left(E_{M} \gg E_{J}, E_{C}\right)$. In the former case $\varphi_{L}$ and $\varphi_{R}$ are not locked to each other and one may still use $k_{L}, k_{R}$ as independent quantum numbers to give a rough construction of the low-energy spectrum. In contrast to the singleisland regime, the oscillator levels are degenerate here [compare Eq. (36) and Eq. (40)]. It is only the parity degree of freedom that is "gapped out" in both regimes.

\section{Regime of four zero-energy $\operatorname{MBS}\left(E_{M}, \varepsilon_{P} \lll E_{C}\right)$}

The system hosts four zero-energy MBS $\gamma_{1}, . ., \gamma_{4}$ as sketched Fig. 1 when both $E_{M}$ and $\varepsilon_{P}$ become negligibly small. Equation (37) shows that the charging-mediated energy splitting $\varepsilon_{P}$ can become exponentially small in $E_{J} / E_{C}$. The lowest-energy parity states $\left|00 ; 0_{12}, 0_{34}\right\rangle$ and $\left|00 ; 1_{12}, 1_{34}\right\rangle\left(k_{L}=k_{R}=0\right)$ are then degenerate up to exponential accuracy. Including also states of odd total parity, the ground state becomes four-fold degenerate and is spanned by $\left|00 ; p_{12}, p_{34}\right\rangle\left(p_{n m}=0,1\right)$.

\section{TIME SCALES FOR GATE-CONTROLLED FUSION-RULE TESTING PROTOCOL}

The segmented nanowire structure shown in Fig. 1 has recently been proposed as en experimental testbed for Majorana physics that could be realized in the near future [55]. MBS may be manipulated by opening and closing junctions through gate control. In this Section, we derive the time-scale conditions (1) and (2) stated in Sec. I. which are required to perform the fusion-rule test suggested in Ref. 55.

The fusion rules of MBS can lead to nontrivial parity correlations by fusing four MBS in complementary pairs. This is rooted in the nonlocal character of the MBS and not possible for local fermions. To prepare and probe such parity correlations, one goes through the steps sketched in Fig. 4(a), changing the parameters along the path in the $\left(E_{J}, E_{M}\right)$ plane as shown in Fig. $4(\mathrm{~b})$. The starting point (A) is to initialize the system in the ground state when the MBS are fused in pairs $\left(\gamma_{2}, \gamma_{3}\right)$ and $\left(\gamma_{1}, \gamma_{4}\right)$. This corresponds to a superposition of eveneven and odd-odd fermion parities in the complementary pairs $\left(\gamma_{1}, \gamma_{2}\right)$ and $\left(\gamma_{3}, \gamma_{4}\right)$. To detect these parity correlations, one first forms all four zero-energy MBS $(\mathrm{C})$ and and then converts them into charge states for the islands (D). Subsequent charge detection then probes the prepared parity correlations. To repeat the experiment by going back from point $\mathrm{D}$ to $\mathrm{A}$, a resetting step is needed, in which the system has to relax into the ground state again.

We now go step by step through the protocol and verify the time-scale criteria (1) and (2). Our considerations 
here concern the cycle sketched in Fig. 4 the time-scale conditions on the readout are discussed in App. E.

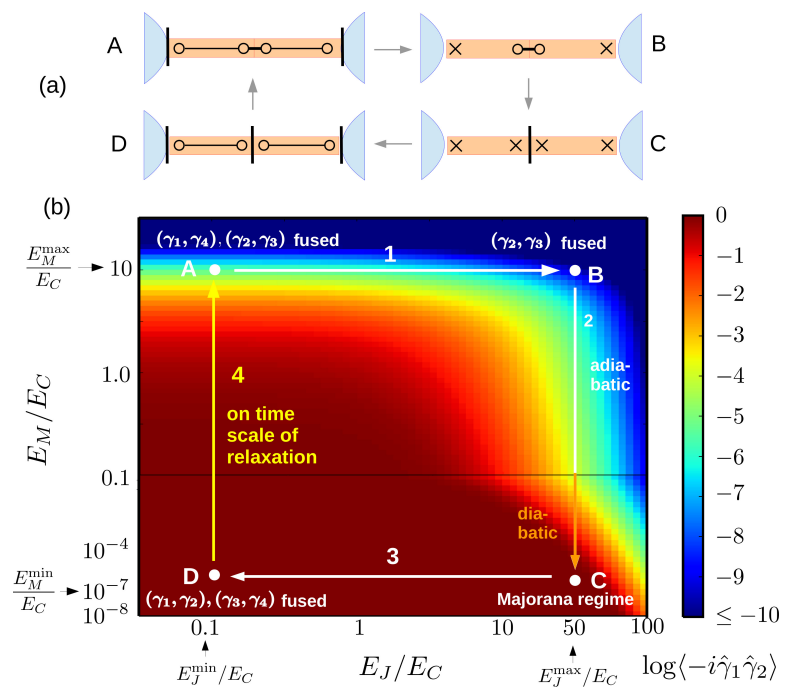

FIG. 4: Protocol for testing the Majorana fusion rules. The steps of the protocol are sketched in (a) and the corresponding path taken in the $\left(E_{J}, E_{M}\right)$ parameter space in (b) (compare with Fig. 2). White arrows indicate that these processes have to be adiabatic, while the orange arrow indicates a diabatic step, in which the system should not follow the ground state evolution. The yellow arrow indicated the resetting step, which should be done on the time scale of the charge relaxation to the ground state. The color scale gives the groundstate expectation value of $\left\langle-i \hat{\gamma}_{1} \hat{\gamma}_{2}\right\rangle$ [see Eq. (46)], indicating the preferred parity combination of the ground state. In the charge-dominated regime $\left(E_{J}, E_{M} \ll E_{C}\right)$, the Majorana pairs $\left(\gamma_{1}, \gamma_{2}\right)$ and $\left(\gamma_{3}, \gamma_{4}\right)$ are fused $\left[\left\langle-i \hat{\gamma}_{1} \hat{\gamma}_{2}\right\rangle \rightarrow 1\right]$, while in the tunneling-dominated regime $\left(E_{M} \gg \varepsilon_{P}\right)$, the Majoranas $\left(\gamma_{2}, \gamma_{3}\right)$ are fused $\left[\left\langle-i \hat{\gamma}_{1} \hat{\gamma}_{2}\right\rangle \rightarrow 0\right]$. To bring all the parity states of the two islands as close to zero energy as possible, the path should cover the Majorana regime $\left(E_{M}, E_{J} \gg E_{C}\right.$ and $E_{M}, E_{J}$ should be, in principle, as large as possible). We use $E_{J, C}=5 E_{M}^{2} / \Delta, E_{J, L}=E_{J, R}=E_{J}, \Delta=100 E_{C}$, $n_{g, R}=-n_{g, L}=0.3$, and $N_{\max }=21$.

\section{A. Time-scale conditions}

To derive the time-scale conditions for each step of the fusion-rule protocol, one has to demand the following adiabaticity condition [71]:

$$
\max _{k, t} f_{k}(t) \ll 1
$$

with

$$
f_{k}(t)=\left|\frac{\left\langle\psi_{k}(t)|\partial H(t) / \partial t| \psi_{0}(t)\right\rangle}{\left[E_{k}(t)-E_{0}(t)\right]^{2}}\right| .
$$

Here, $k$ labels all excited states $\left|\psi_{k}\right\rangle$ at energy $E_{k}$ and 0 labels the ground state $\left|\psi_{0}\right\rangle$ at energy $E_{0}$ [except for step 2, in which the first excited state has to be excluded from condition (43)].

Our first goal is to turn Eq. 43) into a condition for the entire time interval $\Delta t$ when changing the parameters, labeled in the following by $\boldsymbol{\lambda}=\left(\lambda_{1}, \ldots\right)$, from $\boldsymbol{\lambda}(0)$ at time $t=0$ to $\lambda(\Delta t)$ at time $t=\Delta t$. In our case, the parameters are given by $\boldsymbol{\lambda}=\left(E_{J}, E_{M}\right)$. The optimal way to change the parameters is to keep the function $f_{k}(t)$ constant during the parameter sweep because that minimizes the sweeping time $\Delta t$ for a given value of $\max _{k, t} f_{k}(t)$. We can then turn Eq. (43) into the following condition by integrating over time:

$$
\begin{aligned}
\Delta t & \gg \int_{0}^{\Delta t} d t\left|\frac{\left\langle\psi_{k}(t)|\partial H(t) / \partial t| \psi_{0}(t)\right\rangle}{\left[E_{k}(t)-E_{0}(t)\right]^{2}}\right| \\
& =\int_{C} d \boldsymbol{\lambda} \cdot\left|\frac{\left\langle\psi_{k}(\boldsymbol{\lambda})|\partial H(\boldsymbol{\lambda}) / \partial \boldsymbol{\lambda}| \psi_{0}(\boldsymbol{\lambda})\right\rangle}{\left[E_{k}(\boldsymbol{\lambda})-E_{0}(\boldsymbol{\lambda})\right]^{2}}\right| .
\end{aligned}
$$

Here, $C$ is the path in the parameter space connecting $\boldsymbol{\lambda}(0)$ and $\boldsymbol{\lambda}(\Delta t)$. We emphasize that Eq. 43) is sufficient only if $f_{k}(t)$ is constant (in practice remaining of the same order of magnitude), which might correspond to a rather complicated time dependence for $\boldsymbol{\lambda}(t)$. This means that one has to know the properties of the system quite well to design the best gate pulse (in that respect the estimate (45) is optimistic).

Our estimates for the energy gaps $E_{k}(t)-E_{0}(t)$ are based on the numerically computed energy spectrum of Hamiltonian (4) and the analytic approximations worked out in Sec. IV. The gaps to the lowest and further selected excited states along the path shown in Fig. 4 are shown in Fig. 5. We motivate our parameter choices for Figs. 4 and 5 in App. B3. In the following, we work out Eq. (45) only for the lowest accessible excited state and show in App. F that the time scale derived from this is not modified if the effect of transitions into the entire spectrum of higher-lying excited states is included.

Provided optimal pulsing shapes can be achieved, our time-scale estimates are conservative in four respects: (i) We implement the cosine approximation for the Josephson energies in Eq. (4). As we explained in the Appendix of Ref. [55, we expect corrections due to higher harmonics to enhance energy gaps between the ground state(s) and the excited states and to reduce splittings within the ideally degenerate ground-state manifold. The timescale window we estimate here is therefore narrower than what one could expect including these corrections. (ii) While our numerical results in Figs. 4 and 5 include a nonzero Josephson energy $E_{J, C}$ for the central junction, we use $E_{J, C}=0$ for our analytic estimates below. Since nonzero $E_{J, C}$ also enhances the relevant gaps, this again tends to underestimate the actual time-scale window. (iii) For the estimates, we take the islands to be symmetric, $E_{J, L}=E_{J, 2}=E_{J}$, and $E_{C, 1}=E_{C, 2}=E_{C}$. In practice, they will be asymmetric and therefore the energy gaps associated with one of the islands will be less constraining for the time scales than those associated with the other island. (iv) We estimate all matrix elements in Eq. 45 by maximal values (if nonzero) 
even though they can be smaller in practice. We next go through each of the steps of the protocol sketched in Fig. 4 in detail.

\section{B. Initial point}

We start at point $\mathrm{A}$ in the parameter space in Fig. 4(b). Here, the central valve is open $\left(E_{M}=E_{M}^{\max }\right)$, while the valves to the bulk superconductors are closed $\left(E_{J}=E_{J}^{\min }\right)$. In this configuration, the MBS pairs $\left(\gamma_{2}, \gamma_{3}\right)$ and $\left(\gamma_{1}, \gamma_{4}\right)$ are fused. In the ground state, $\left|N=0, k_{\Delta}=0 ; 0_{14} 0_{23}\right\rangle$, the corresponding fermionic modes are empty and no Josephson-plasma oscillations are excited. We will from hereon suppress quantization indices for charge state / plasma oscillations whenever we refer to the lowest state with respect to these degrees of freedom, i.e., $\left|p_{14} p_{23}\right\rangle=\left|N=0, k_{\Delta}=0 ; p_{14} p_{23}\right\rangle$.

To illustrate the evolution of the parity of the ground state along the protocol, we show with the color scale in Fig. 4(b) the numerically computed ground-state expectation value of the operator

$$
\begin{aligned}
& -i \hat{\gamma}_{1} \hat{\gamma}_{2}=\hat{P}_{e e}-\hat{P}_{o o} \\
& =\left(\sum_{n_{L}, n_{R} \text { even }}-\sum_{n_{L}, n_{R} \text { odd }}\right)\left|n_{L} n_{R}\right\rangle\left\langle n_{L} n_{R}\right| .
\end{aligned}
$$

If $\left\langle-i \hat{\gamma}_{1} \hat{\gamma}_{2}\right\rangle=+1(-1)$, a state has even-even (odd-odd) parity, while if $\left\langle-i \hat{\gamma}_{1} \hat{\gamma}_{2}\right\rangle=0$, the state is a linear combination of an even-even and an odd-odd state with equal probability (we restrict ourselves here to the subspace of even total parity). Figure 4(b) demonstrates that, at point $\mathrm{A},\left|\left\langle-i \hat{\gamma}_{1} \hat{\gamma}_{2}\right\rangle\right| \ll 1$ in the ground state, which is consistent with the form $\left|0_{14} 0_{23}\right\rangle=\left[\left|0_{12} 0_{34}\right\rangle+\right.$ $\left.\left|1_{12} 1_{34}\right\rangle\right] / \sqrt{2}$.

\section{Step 1: Initialization of $\gamma_{1}$ and $\gamma_{4}$}

The first step of the protocol brings the MBS $\gamma_{1}$ and $\gamma_{4}$ located at the two outer ends of the island close to zero energy 93 . This is achieved by opening the valves to the bulk superconductors $\left[\lambda=E_{J} \rightarrow E_{J}^{\max }\right.$ at point $\mathrm{B}$ in Fig. 4(b)], which suppresses charging effects on both islands. Yet, the Majorana pair $\left(\gamma_{2}, \gamma_{3}\right)$ remains fused, i.e., $\left\langle-i \hat{\gamma}_{1} \hat{\gamma}_{2}\right\rangle$ remains essentially zero along the curve $A \rightarrow B$ in Fig. 4(b). The numerically computed energy gap $E_{1}-E_{0}$ between the first excited state and the ground state $\left|0_{14} 0_{23}\right\rangle$ is shown in Fig. 5 as a function of $E_{J}$.

Concerning the time-scale $T_{1}$ for this step, we can use $\left|\left\langle\psi_{1}\left|\partial H / \partial E_{J}\right| \psi_{0}\right\rangle\right| \leqslant 1$ and express Eq. (45) as

$$
T_{1} \gg \int_{0}^{E_{C}} \frac{d E_{J}}{\left|E_{1}-E_{0}\right|^{2}}+\int_{E_{C}}^{E_{J}^{\max }} \frac{d E_{J}}{\left|E_{1}-E_{0}\right|^{2}} .
$$

We decomposed the integral into two parts according to the two parameter regimes crossed during the $E_{J}$ sweep. For $E_{J} \lesssim E_{C}$, the gap is dominated by the charging energy and we can estimate $E_{1}-E_{0} \geq 2 E_{C}$. For $E_{C} \lesssim$ $E_{J}<E_{J}^{\max }<\left(E_{M}^{\max }\right)^{2} / 2 E_{C}$, the gap is dominated by Josephson plasma oscillations and given roughly by $E_{1}-$ $E_{0} \approx \sqrt{8 E_{J} E_{C}}$ as the light blue dashed line in Fig. 5 illustrates. Inserting these two estimates into Eq. (47), we obtain

$$
T_{1} \gg \frac{\ln \left(E_{J}^{\max } / E_{C}\right)}{E_{C}} .
$$

where we used $E_{J}^{\max } / E_{C} \gg 1$. We see that the timescale condition scales only logarithmically with $E_{J}^{\max }$. Accounting for the dependence of the matrix elements $\left|\left\langle\psi_{1}\left|\partial H / \partial E_{J}\right| \psi_{0}\right\rangle\right|$ on $E_{J}$ might result in even less restrictive conditions than Eq. (48) 94. However, for the experimental situation we have in mind, the ratio $E_{J}^{\max } / E_{C}$ will be a few tens and the logarithmic term is less important.

We finally mention that condition $(48)$ has to be reconsidered if $E_{J}^{\max } \gg\left(E_{M}^{\max }\right)^{2} / 2 E_{C}$ : The gap to the first excited state is then constant and given by $2 E_{M}^{\max }$. However, transitions into this lowest state are forbidden (see below) and for the parameters used Fig. 5, one hardly enters into this regime.

\section{Step 2: Initialization of $\gamma_{2}$ and $\gamma_{3}$}

Closing the central valve brings the Majoranas $\gamma_{2}$ and $\gamma_{3}$ to zero energy $\left[E_{M} \rightarrow E_{M}^{\min }\right.$ at point C in Fig. 4(b)]. This separates the system into two decoupled islands and the two states $\left|0_{14} 0_{23}\right\rangle,\left|1_{14} 1_{23}\right\rangle=\left[\left|0_{12} 0_{34}\right\rangle \pm\left|1_{12} 1_{34}\right\rangle\right] / \sqrt{2}$ become degenerate, at least ideally. As in any other topological setup, the ground-state degeneracy is in practice slightly broken due to the charging-induced parity splitting $\varepsilon_{P}$ on each of the islands (see Sec. IVC). This splitting is suppressed with exponential accuracy but renders the even-even parity state $\left|0_{12} 0_{34}\right\rangle$ the ground state for $E_{M} \ll \varepsilon_{P}$. Therefore, this step has to be performed diabatically regarding the two lowest states when $E_{M}$ becomes of the order of $\varepsilon_{P}$. The goal is here that the system remains in the prepared superposition $\left|\psi_{0}\right\rangle=\left(\left|0_{12} 0_{34}\right\rangle+\left|1_{12} 1_{34}\right\rangle\right) / \sqrt{2}$. Moreover, this step should be adiabatic regarding all other excited states, which is the condition we discuss first.

Adiabaticity condition. For $E_{M} \gg \varepsilon_{P}$, the tunnel splitting $2 E_{M}$ sets the gap $E_{1}-E_{0}$ between the ground and first excited state (red line in Fig. 5). Importantly, the tunneling Hamiltonian $H_{T}$ does not allow for transitions between the two lowest states $\left|0_{14} 0_{23}\right\rangle$ and $\left|1_{14} 1_{23}\right\rangle$ because $H_{T}$ cannot flip the nonlocal parities of the fermionic modes $\hat{f}_{14}=\hat{\gamma}_{1}+i \hat{\gamma}_{4}$ and $\hat{f}_{23}=\hat{\gamma}_{3}+i \hat{\gamma}_{3}$. The lowest accessible excited state is therefore $\left|\psi_{2}\right\rangle \approx$ $\left|k_{L}+k_{R}=1 ; 0_{14} 0_{23}\right\rangle$ with an excited Josephson plasma oscillation on one of the islands as explained in Sec. IV C (ii). The energy gap is constant here: $E_{2}-E_{0} \sim$ 


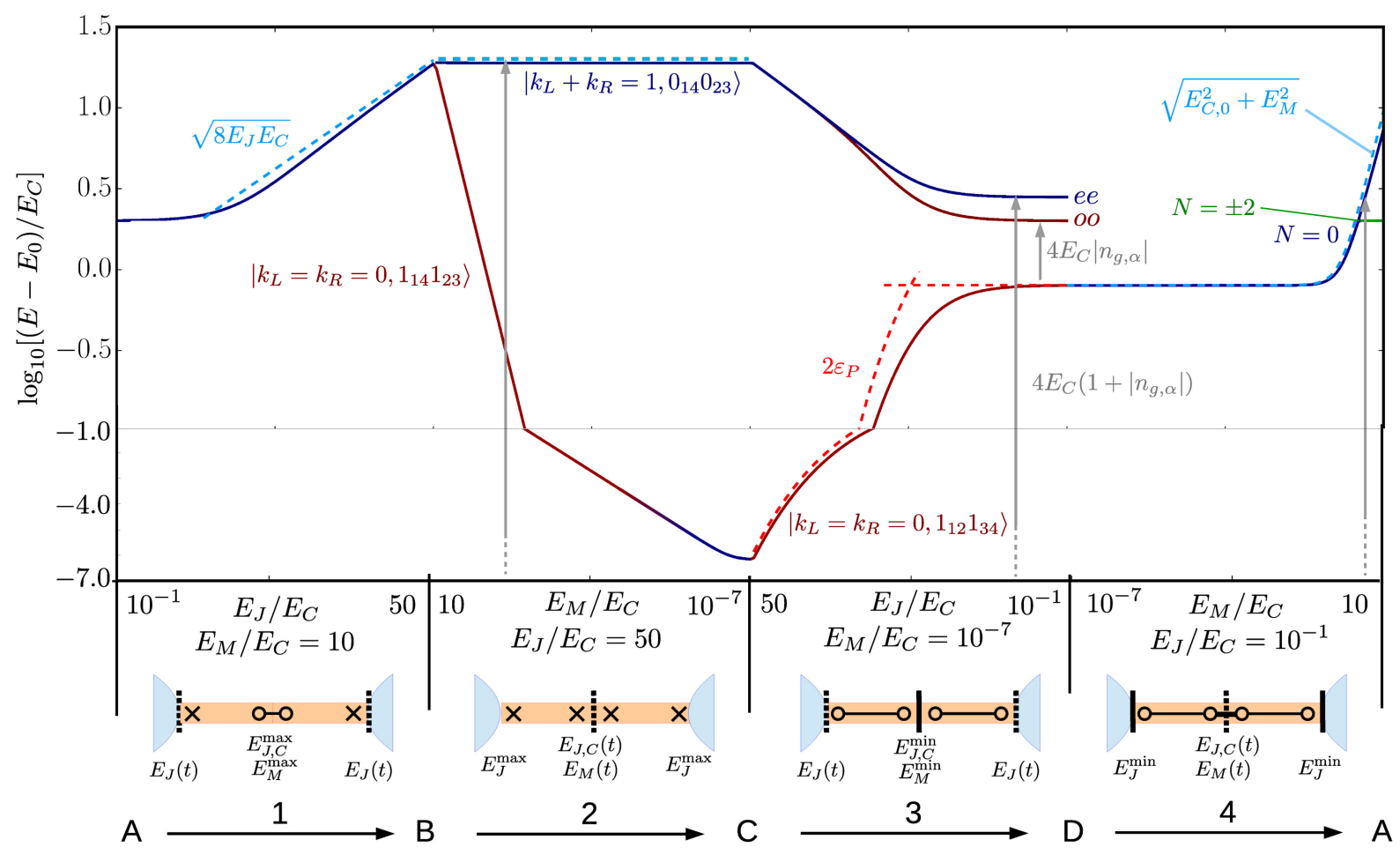

FIG. 5: Energy gap between the ground state and first excited state for even total parity (in some intervals the gap to other excited states is shown as well). The parameters $E_{M}$ (and accordingly $E_{J, C}=5 E_{M}^{2} / \Delta$ ), as well as $E_{J}$ on the horizontal axis are changed logarithmically along the path $A \rightarrow B \rightarrow C \rightarrow D \rightarrow A$ marked in Fig. 4(b). The solid lines correspond to the numerically computed splittings while the dashed lines are the approximation formulas for the splittings (see text). Gray arrows indicate allowed transitions and we mark the lowest accessible excited state from the ground state in blue. In several steps transitions into the lowest excited states (red, green) are prohibited by parity or charge conservation as explained next: In step 2, the lowest excited state $\left|1_{23}, 1_{14}\right\rangle$ is decoupled from the ground state $\left|0_{23}, 0_{14}\right\rangle$ for a large energy range because the tunneling Hamiltonian $H_{T}$ conserves the nonlocal parities $p_{23}, p_{14}$. However, when $\varepsilon_{P}$ becomes dominant, the parity character of the ground state changes to $\left|0_{12} 0_{34}\right\rangle$. Thus, when $E_{M}$ approaches $\varepsilon_{P}$, transitions to the lowest excited state become possible. By tuning fast the system stays in the desired state $\left|0_{23}, 0_{14}\right\rangle=\left(\left|0_{12} 0_{34}\right\rangle+\left|1_{12} 1_{34}\right\rangle\right) / \sqrt{2}$, i.e., the system can be both in the ground and first excited state after step 2. Since the islands are decoupled then, the local parities $p_{12}, p_{34}$ are conserved in step 3 and transitions from even-even to odd-odd parity or vice versa are prohibited. From the ground state, one can therefore only reach the third-lowest excited state [blue, parity $\left(0_{12}, 0_{34}\right)$ ]. In addition, transitions from the first to the second excited state are possible [red, parity $\left(1_{12}, 1_{34}\right)$ ], which determines the adiabaticity condition. Finally, in step 4 , the islands host a different total charge $N= \pm 2$ in the lowest excited state, while transitions are only possible into the lowest $N=0$ state $\left(N=n_{L}+n_{R}\right.$ is the total number of electrons). The parameters are $n_{g, R}=-n_{g, L}=0.3, \Delta=100 E_{C}, E_{C, 0}=2 E_{C}\left(1+n_{g, L}-n_{g, R}\right)(\mathrm{see}$ text), and the number-state cutoff is $N_{\max }=25$. Note that we chose a rather large value for $\Delta$ to be consistent with the assumption $E_{C} \ll E_{M}^{\max } \ll \Delta$ for our numerical approach. We expect that the protocol should also work for smaller values of $\Delta$ as we further explain in App. B

$\sqrt{8 E_{J}^{\max } E_{C}}$ (blue dashed line in Fig. 5). Inserting this into Eq. 45 and using $\left|\left\langle\psi_{k}\left|\partial H / \partial E_{M}\right| \psi_{0}\right\rangle\right| \leqslant 1$ yields the condition:

$$
T_{2} \gg \frac{E_{M}^{\max }}{8 E_{J}^{\max } E_{C}},
$$

If $E_{M}^{\max } / E_{J}^{\max }<1$, as assumed here, this condition is fulfilled if $T_{2} \gg 1 / E_{C}$, which is a looser condition than Eq. 48.

Diabaticity condition. If $E_{M} \ll \varepsilon_{P}$, the two lowest eigenstates are $\left|0_{12} 0_{34}\right\rangle$ and $\left|1_{12} 1_{34}\right\rangle$ and split by $2 \varepsilon_{P}$ as discussed in Sec. IV C (i). The system has to evolve diabatically when tuning through the crossover point at
$E_{M}=\varepsilon_{P}$, i.e., step 2 has be carried out fast in the sense that

$$
T_{2} \ll \frac{1}{\varepsilon_{P}^{\min }},
$$

where $\varepsilon_{P}^{\min }$ is the value for $\varepsilon_{P}$ at point $\mathrm{C}$, where it is minimal because $E_{J} / E_{C}$ is maximal [see Eq. (37)]. Roughly speaking, the above condition ensures that the timeevolution operator with the ground-state manifold can be approximated as 


$$
\begin{aligned}
U\left(T_{2}\right)= & e^{-i \int_{0}^{T_{2}} d t H(t)}=e^{-i \int_{0}^{T_{2}} d t \varepsilon_{P}(t)}\left|1_{12} 1_{34}\right\rangle\left\langle 1_{12} 1_{34}\right| \\
& +e^{+i \int_{0}^{T_{2}} d t \varepsilon_{P}(t)}\left|0_{12} 0_{34}\right\rangle\left\langle 0_{12} 0_{34}\right| \approx 1 .
\end{aligned}
$$

\section{E. Step 3: Parity-to-charge conversion}

The next step is to read out the fermion parities of each of the disconnected islands, which necessitates closing of the valves to the bulk superconductors again $\left[E_{J} \rightarrow E_{J}^{\min }\right.$ at point D in Fig. 4(b)]. The two decoupled parity components in the prepared state $\left|0_{14} 0_{23}\right\rangle=$ $\left(\left|0_{12} 0_{34}\right\rangle+\left|1_{12} 1_{34}\right\rangle\right) / \sqrt{2}$ are thereby mapped onto different charge states. We assume for the following estimations that the gate voltages are adjusted such that

$$
-1 / 2<n_{g, L}<0<n_{g, R}<1 / 2,
$$

and that the $n_{g, \alpha}$ are not close to either of the boundaries. Under this assumption, the parity states are transferred to 95

$$
\begin{aligned}
& \left|0_{12} 0_{34}\right\rangle \rightarrow\left|n_{L}=0, n_{R}=0\right\rangle, \\
& \left|1_{12} 1_{34}\right\rangle \rightarrow\left|n_{L}=-1, n_{R}=+1\right\rangle .
\end{aligned}
$$

These charge states are subsequently detected either by proximal charge sensors or by a charge pumping scheme. The details of these two readout schemes are explained in Ref. [55] and the time-scales for the charge pumping are considered further in App. E, Note that the above scheme also works for asymmetric charging energies or in the presence of cross-capacitive couplings between the islands; however, the conditions on the gatings may be altered.

Adiabaticity condition. The adiabaticity criteria for step 3 are closely related to those of step 1 . The only difference is that we have to consider the subspaces of even-even and odd-odd parity separately. They are decoupled because the two wire segments are decoupled $\left(E_{M}=E_{M}^{\min }\right)$. For $E_{J} \gg E_{C}$, the system behaves as two decoupled superconducting islands and the gaps in both parity sectors are the same: $E_{1, \text { ee }}-E_{0, \text { ee }}=$ $E_{1, \mathrm{oo}}-E_{0, \mathrm{oo}} \approx \sqrt{8 E_{J} E_{C}}$. For $E_{J} \ll E_{C}$ the system behaves as two decoupled topological Cooper pair boxes. Here the eigenstates are close to charge states and the gaps between them depend sensitively on the gating: Under condition (52), we get $E_{1, \text { ee }}-E_{0, \text { ee }}=4 E_{C}\left(1+n_{g, L}\right)$, while $E_{1, \text { oo }}-E_{0, \text { oo }}=4 E_{C} \min \left(\left|n_{g, L}\right|,\left|n_{g, R}\right|\right)$. Provided $\left|n_{g, \alpha}\right|=O(1)$, both gaps are on the order of the charging energy. We can thus follow the argumentation of step 1 and obtain the following adiabaticity condition:

$$
T_{3} \gg \frac{\ln \left(E_{J}^{\max } / E_{C}\right)}{E_{C}} .
$$

No-relaxation condition. Clearly, this operation has to be done fast enough so that no electrons can be exchanged between the two islands, i.e.,

$$
T_{3} \ll \frac{1}{E_{M}^{\min }} .
$$

Otherwise a leakage into the even-even ground state may spoil the readout.

\section{F. Step 4: Reset}

The final step is to close the cycle in parameter space in order to repeat the protocol again. Opening the central valve $\left(E_{M} \rightarrow E_{M}^{\max }\right.$ at point A) fuses the MBS pair $\left(\gamma_{2}, \gamma_{3}\right)$ and brings the parameters of the system back to the initial point.

Relaxation condition. Depending on the measurement outcome, the reset requires a relaxation process because the odd-odd parity configuration is an excited state of the device. Before repeating the cycle, one thus has to wait until the system has relaxed to the ground state (which is possible if the central valve is opened):

$$
T_{4} \gg \tau_{\text {relax }} \text {. }
$$

We estimate the involved charge relaxation processes by typical charge relaxation times for GaAs double quantum dots, which are on the order of 10 ns [72, 73] due to phonon emission [96. However, the relaxation time scales for nanowire setups in the presence of a screening superconductor might be different and an interesting future task to investigate. Since such a relaxation process is needed anyway, there is also no general reason to perform the parameter sweep adiabatically, at least in the charge-readout scheme. For the charge readout, one can simply wait long enough after opening the central valve until the relaxation has certainly happened.

Adiabaticity condition. For the pumping readout scheme, it would be desirable to perform the resetting step fast as possible in order to maximize the pumping current. Besides waiting for charge relaxation, it is therefore favorable to open the central valve adiabatically to make sure that the system is not driven to even higher excited states (necessitating possibly multiple relaxation steps). For this reason, and because we use it later in Sec. VIA 1, we investigate here the conditions needed to perform the opening of the central valve adiabatically.

As mentioned in Sec. IVB the Hamiltonian conserves the total number of electrons $N=n_{L}+n_{R}$ on the islands when the junctions to the bulk superconductors are closed. Therefore, transitions are possible only within the sectors for fixed $N$. Using the inequality $\left|\left\langle\psi_{1, N=0}\left|\partial H / \partial E_{M}\right| \psi_{0}\right\rangle\right| \leqslant 1$, we obtain from Eq. 45) the sufficient condition:

$$
T_{4} \gg\left(\int_{0}^{E_{C}}+\int_{E_{C}}^{E_{M}^{\max }}\right) \frac{d E_{M}}{\left|E_{1, N=0}-E_{0}\right|^{2}},
$$

where we have split the integral again into two parts analogous to our considerations in step 1 . For $E_{M} \lesssim E_{C}$, the 
gap is dominated by the charging energy (blue-dashed line in Fig. 5f: $E_{1}-E_{0} \approx \sqrt{E_{C, 0}^{2}+E_{M}^{2}} \geqslant E_{C, 0}:=$ $2 E_{C}\left(1+n_{g, L}-n_{g, R}\right) \sim E_{C}$ under condition (52). For $E_{M} \gg E_{C}$, the system is tuned into the single island regime considered in Sec. IV B and the gap can be estimated by $E_{1, N=0}-E_{0} \approx \sqrt{4 E_{C} E_{M}}$ for $E_{J, C}=0$. The latter regime is not reached for the parameters used in Fig. 5. Inserting these two gap estimates into the above integrals, we get the condition

$$
T_{4} \gg \frac{\ln \left(E_{M}^{\max } / E_{C}\right)}{E_{C}},
$$

for $E_{M}^{\max } / E_{C} \gg 1$ and assuming that $n_{g, L}-n_{g, R}$ does not come close to 1 .

This finally completes the account of the time-scale conditions (1) and (2) for the fusion-rule testing protocol.

\section{G. Time-scale estimate for entire cycle}

According to the above estimates, all steps are adiabatic if carried out on a time scale $\sim 1 / E_{C}$. For charging energies of a few hundred $\mathrm{mK}$, which is much smaller than the typical superconducting gap in $\mathrm{Al}$ (around $2 \mathrm{~K}$ [45]), we obtain $1 / E_{C} \sim 0.1$ ns. Taking all the steps and logarithmic correction factors into account, steps 13 have to be carried out on a time scale of $10 \mathrm{~ns}$ to be adiabatic. This is a time scale on the same order as typical times for charge relaxation needed in the resetting step [72, 73. To be sure the system has completely relaxed into the ground state, $100 \mathrm{~ns}$ for the entire cycle in the pumping scheme seems reasonable. This results in a pumping current of a few pA.

We have so far not considered the time-scale conditions for the readout, which we postpone to App. E. We discuss there that a parity-selective pumping process may be implemented on a time scale faster than $\sim 1 / E_{C}$, i.e., the steps for the pumping play a minor role in estimating the minimal cycle period.

\section{TIME SCALES FOR GATE-CONTROLLED MAJORANA MANIPULATIONS IN NANOWIRE NETWORKS}

In this final section, we estimate time scales for performing gate-controlled exchanges of MBS, which requires going from single-wire structures as sketched in Fig. 6(a) to branched structures such as trijunction setups as depicted in Fig. 6(b). We envisage the three topological wire segments (orange) to be connected via a nontopological region (blue), which may be either normal or superconducting.

We specifically work out two basic operations that are needed for realizing braiding: We first consider in Sec. VIA the transfer of a MBS from one nanowire segment to another as sketched in Fig. 6(c). The second operation, considered in Sec. VIB, is the transfer of a MBS across a trijunction as sketched in Fig. 6(d). While the first operation can be analyzed from simulations of the segmented nanowire device, the second operation would, in general, require a numerical simulation of a trijunction geometry, which we do not pursue here. However, with our insights from Sec. IV] for the single-wire geometry, we may identify the relevant energy scales for the trijunction geometry, allowing us to give a conservative time-scale estimate also for the operations in nanowire networks. By concatenating operations of Figs. 6(c) and (d), it is possible to exchange two MBS as shown in Fig. 6(e). This realizes the braiding protocol discussed in Ref. 55, closely related to Refs. 34, 37, 41 but using electrical control over the Josephson couplings as an alternative to magnetic flux manipulation schemes.

\section{A. Transfer of Majorana bound states across coupled nanowire segments}

We first discuss the transfer of a MBS from one nanowire segment to the other as sketched in Fig. 6(c). To be specific, let us first consider the situation when (A) the valve to the left bulk superconductor is maximally open $\left(E_{J, L} \gg E_{C, L}\right)$ and all other valves are closed $\left(E_{M}=E_{J, C}=E_{J, R}=0\right)$. Then both MBS $\gamma_{1}$ and $\gamma_{2}$ on the left island are very close to zero energy (the energy splitting is exponentially small in $\left.\sqrt{E_{J, L} / E_{C, L}}\right)$. By contrast, the MBS $\gamma_{3}$ and $\gamma_{4}$ on the right island are fused due to their coupling through the charging energy $E_{C, R}$. To transfer the MBS $\gamma_{2}$ at the central junction to the right wire segment, one first opens the central valve, going through the configuration $(\mathrm{B})$ to $(\mathrm{C})$ and then opens the valve to the right bulk superconductor resulting in (D).

We emphasize that the order of opening the valves is important: Opening the right valve first would increase the ground-state degeneracy by bringing $\gamma_{3}$ and $\gamma_{4}$ to zero energy resulting in the configuration shown in Fig. 6(a). Opening the central valve could then lead to uncontrolled rotations in the ground-state subspace, which must be avoided. If instead the central valve is opened first, the ground state degeneracy remains two-fold (including both even and odd total parity). To illustrate this point, let us consider the following Majorana Hamiltonian,

$$
H=i E_{M} \hat{\gamma}_{2} \hat{\gamma}_{3}+i \varepsilon_{P, R} \hat{\gamma}_{3} \hat{\gamma}_{4}
$$

accounting for the charging-induced coupling $\varepsilon_{P, R}$ of MBS $\gamma_{3}$ and $\gamma_{4}$ and the tunnel coupling $E_{M}$ of MBS $\gamma_{2}$ and $\gamma_{3}$. Diagonalization shows that fusing three MBS yields one fermionic mode at finite energy and one MBS, $\gamma_{0}$, that remains at zero energy [42]:

$$
\hat{\gamma}_{0}=a \hat{\gamma}_{2}+b \hat{\gamma}_{4}
$$


(a)
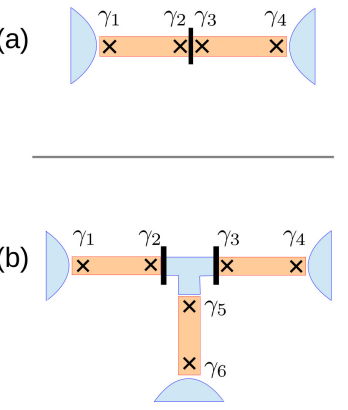

(c)

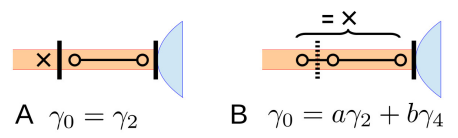

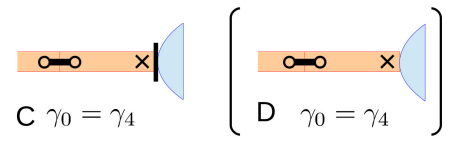

(d)

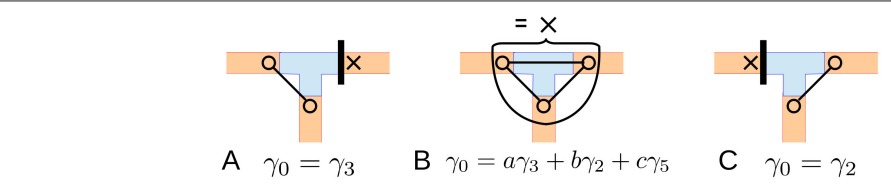

A $\gamma_{0}=\gamma_{3}$

B $\gamma_{0}=a \gamma_{3}+b \gamma_{2}+c \gamma_{5}$

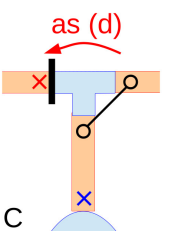

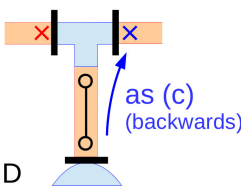

FIG. 6: Basic operations for gate-controlled manipulations of MBS in nanowire networks. (a) and (b) show the positions of the four and six MBS in the segmented and trijunction nanowire geometry, respectively. In (c), we show the steps of transferring a MBS from one end of the left wire segment to the opposite end of the right segment. During the entire transfer process, a MBS $\gamma_{0}$ remains at zero energy (see Eq. (61) and the explanation below), which is a linear combination with changing weights of $\gamma_{2}$ and $\gamma_{4}$ as indicated in the figure. We put the last step in parenthesis since opening the valve to the right superconductor is not necessary, provided the coupling of the central junction can be made large enough (as specified in Sec. VI A). In (d), we show the steps to transfer a MBS from the right side of the trijunction to the left side. (e) Elementary braid operation, composed of operations (c) and (d). Analogous to (c), the vertical island needs not be connected to a bulk superconductor for large couplings across the trijunction. We emphasize that all operations in (c) [(d) and (e)] must be performed such that the ground-state degeneracy is not changed during the operation, meaning that during the braid operation the system should never be in the configurations shown in (a) [(b)] with 4 (6) uncoupled MBS.

The MBS operator $\hat{\gamma}_{3}$ does not appear in Eq. 61) because MBS $\gamma_{2}$ and $\gamma_{4}$ are not directly coupled. Equation (61) implies that irrespective of the values for $E_{M}$ and $E_{J, R}$, which control the coefficients $a$ and $b$, the ground state is always two-fold degenerate with respect to the fermionic mode $\hat{f}=\hat{\gamma}_{1}+i \hat{\gamma}_{0}$. To verify this, we numerically computed the energy spectrum of Hamiltonian (4) for both total even parity as well as total odd parity. We have verified that the energy splitting between the lowest states in both sectors is given by Eq. (37) for $E_{J, L} / E_{C} \gg 1$, that is, it is exponentially small in $\sqrt{E_{J, L} / E_{C}}$.

We note that the Majorana Hamiltonian $(60)$ is a good effective description only for the regime $E_{C} \ll E_{M} \ll$ $E_{J, L}$, as it otherwise fails to describe the relevant energy gaps otherwise because it ignores the Cooper pair condensate. (The validity is further discussed in App. G.) The parameter $\varepsilon_{P, R}$ depends, in principle, on all other parameters and can be suppressed with increasing $E_{M}$. In general, the energy gaps have to be inferred from the full Hamiltonian.

\section{Time-scale conditions}

Based on the adiabaticity criterion (45), we next show that the transfer proceeds adiabatically if it is carried out on a time scale

$$
T \gg \frac{\ln \left[\max \left(E_{J, R}^{\max }, E_{M}^{\max }\right) / E_{C}\right]}{E_{C}},
$$

provided $E_{M}^{\max }, E_{J, R}^{\max } \leq E_{J, L}^{\max }$. This is a conservative estimate derived by assuming $E_{J, C}=0$. In Fig. 7, we show the energy gap between the lowest excited and the ground state for both steps of the MBS transfer, both including and excluding the central Josephson coupling $E_{J, C}$. Our results are again restricted to even total parity (transitions into states of different total parity are forbidden). We next discuss the time scales for each of the two steps.

Step 1: Opening the central valve $\left(E_{M} \rightarrow E_{M}^{\max }\right)$. This step is related to the parameter sweep performed in step 4 in the fusion-rule protocol. Here, we are, however, only interested in the adiabatic evolution of the ground state and the total charge on the two islands is not conserved because $E_{J, L} \neq 0$. Thus, transitions are also possible into the lowest excited state. However, the condition $E_{J, L} \gg E_{C}$ also implies that the smallest energy gap is not independent of $E_{M}$ as in the case of $E_{J, L}=0$. In fact, by comparing Fig. 7 with Fig. 5 we can see that the lowest excited state for $E_{J, L} \gg E_{C}$ (Fig. 7) follows the same $E_{M}$ dependence as the lowest state in the $N=0$ sector for $E_{J, L}=0$ (step 4 in Fig. 5). We can thus follow the same considerations as for step 4 in the fusion-rule protocol and obtain the following sufficient condition for adiabatic evolution:

$$
T_{1} \gg \frac{\ln \left(E_{M}^{\max } / E_{C}\right)}{E_{C}} .
$$




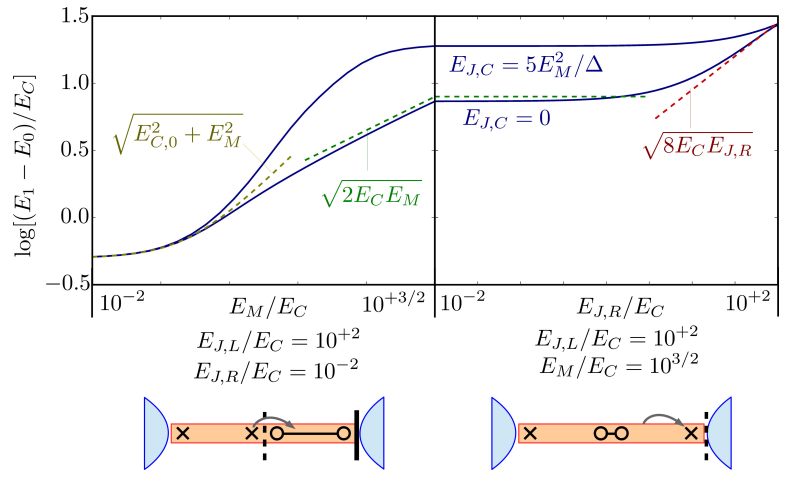

FIG. 7: Transfer of MBS between two nanowire segments. Numerically computed energy gap (solid blue) between the ground state and the first excited state. The two curves are for two different values of $E_{J, C}$. We change $E_{M}$ in the left part and $E_{J, R}$ in the right part as illustrated in the sketches below the plots. We also show analytic approximations for the energy splitting (dashed lines) as denoted and further discussed in the text. Due to the gating of the right island, the relevant charging energy is given by $E_{C, 0}=E_{C}\left(1-2 n_{g, R}\right)$. The other parameters are given by $E_{C, L}=E_{C, R}=E_{C}, n_{g, L}=0$, $n_{g, R}=1 / 4$, and $N_{\max }=20$.

We note that an underlying assumption here is that $E_{M}^{\max }<E_{J, L}$. Otherwise the two islands are strongly hybridized and the lowest excited state is the first excited state of the Josephson plasma oscillations [see Sec. IV B (B)]. This regime is not reached for the parameters we assume in Fig. 7 .

Step 2: Opening the right valve $\left(E_{J, R} \rightarrow E_{J, R}^{\max }\right)$. As the right half of Fig. 7 shows, the energy gap $E_{1}-E_{0} \sim \sqrt{2 E_{J} E_{M}}$ remains constant over a larger range and increases only for larger $E_{J, R}$. If the Josephson coupling to right bulk superconductor in the final stage does not exceed that to left bulk superconductor (i.e., $\left.E_{J, R}^{\max } \leqslant E_{J, L}^{\max }\right)$, the gap $\Delta E$ satisfies

$$
\Delta E\left(E_{J, L}^{\max }, E_{J, R}\right) \geqslant \Delta E\left(E_{J, L}=E_{J, R}\right)
$$

i.e., the gap is always larger than that given when symmetrically increasing both Josephson couplings. The symmetric increase is studied in Sec. $\mathrm{VC}$ for step 1 of the fusion-rule protocol and the adiabaticity criterion (43) should be satisfied here even better due to the larger gap. Employing the result 48 given there, the evolution proceeds adiabatically if the time for this step satisfies

$$
T_{2} \gg \frac{\ln \left(E_{J, R}^{\max } / E_{C}\right)}{E_{C}} .
$$

Conditions 63 and (64) show that transferring MBS between different wire segments can be performed on the same time scale as initializing them for, e.g., testing the fusion rules as discussed in Sec. V.

\section{Suppression of charging effects through the center junction}

We next show that the above transfer of the MBS between two segments can actually be achieved even without the second step of opening the right valve to the bulk superconductor. A prerequisite is that the condition $E_{C} \ll E_{M}, E_{J, L}$ can be satisfied.

Intuitively, one could expect that the connection to the right bulk is not needed once the system behaves as larger superconducting island for large Majorana coupling $E_{M}>E_{J}$. We verify this expectation in App. G. where we show that asymmetries of the bulk Josephson couplings $E_{J, \alpha}$ become irrelevant for $E_{M} \gg E_{J}$. However, it turns out that the condition $E_{M}>E_{J}$ is, in fact, not necessary at all.

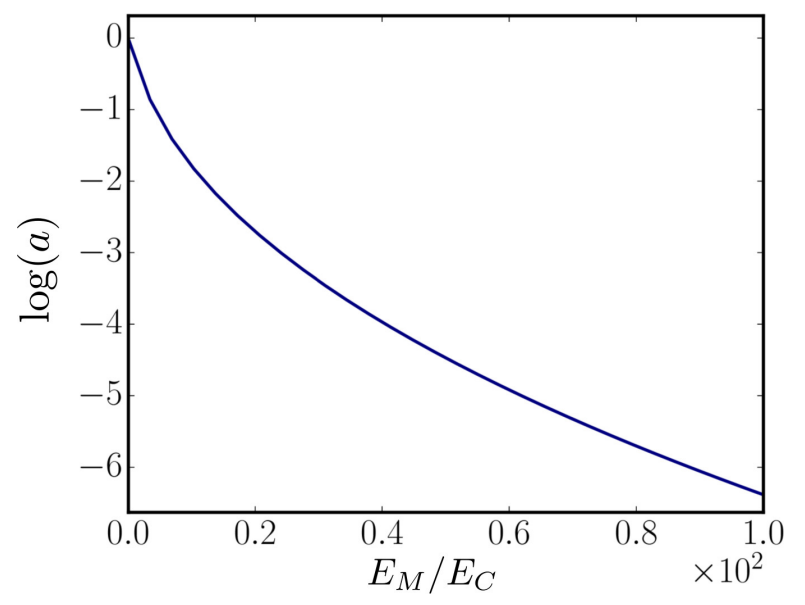

FIG. 8: Gate-controlled transfer of a MBS between two nanowire segments. We show the numerically computed coefficient $a$ in Eq. 61) as a function of the Majorana coupling $E_{M}$. The calculation of the coefficient $a$ is explained in App. G. The parameters used here are $E_{J, L}=100 E_{C}$, $E_{J, R}=E_{J, C}=0, n_{g, L}=0, n_{g, R}=0.25$, and $N_{\max }=10$.

To demonstrate this, we show the dependence of the coefficient $a$ in the linear combination $\hat{\gamma}_{0}=a \hat{\gamma}_{2}+b \hat{\gamma}_{4}$ [Eq. (61)] in Fig. 8. Indeed, we find that $a$ is strongly suppressed as a function of the Majorana tunneling $E_{M}$ (exponentially for very large ratios $E_{M} / E_{C}$ ). The technical details how we extract $a$ and $b$ from our numerical simulations are postponed to App. G. The exponential suppression of $a$ crucially relies on a suppression of the coupling $\varepsilon_{P, R}$ between the two MBS on the right island. In other words, tuning the Majorana coupling changes not only the parameter $E_{M}$ in the effective model 60, but also the coupling $\varepsilon_{P, R}$. This demonstrates that it is important to include the dynamics of the Cooper pair condensates on the islands to arrive at an effective description of the Majorana physics. 


\section{B. Transfer of Majorana bound states across trijunctions}

The second operation we consider is the transfer of a MBS across a trijunction as sketched in Fig. 6(d). At first, the left valve at the trijunction is open, connecting the left and the vertical segment, while the right valve at the trijunction is closed as shown in (A). A MBS is thus located at the right side of the trijunction. Then the right valve is opened and all three MBS located near the branching point are fused as depicted in (B). Analogous to the MBS transfer discussed above, this leaves behind a finite-energy fermionic mode and one zero-energy MBS, which is a linear combination of all three coupled MBS. Following the notation introduced in Fig. 6(b), this mode can be expressed as

$$
\hat{\gamma}_{0}=a \hat{\gamma}_{2}+b \hat{\gamma}_{3}+c \hat{\gamma}_{5}
$$

Finally, the left valve is closed and the MBS is located at the left of the trijunction, while now the right and vertical segments are connected as shown in (C).

To estimate time scales for this transfer process, we first need to specify a model for the trijunction geometry. We assume that the Hamiltonian is a simple extension of the two-fold segmented nanowire structure [42]:

$$
\begin{aligned}
H= & \sum_{\alpha=L, R, V} E_{C, \alpha}\left(\hat{n}_{\alpha}-n_{g, \alpha}\right)^{2}+E_{J, \alpha}\left(1-\cos \left(\hat{\varphi}_{\alpha}\right)\right) \\
& +\sum_{\langle\alpha, \beta\rangle} E_{M, \alpha \beta} \cos \left(\frac{\hat{\varphi}_{\alpha}-\hat{\varphi}_{\beta}}{2}\right) i \hat{\gamma}_{\alpha} \hat{\gamma}_{\beta} .
\end{aligned}
$$

In the second term above, we sum over the pairs $\langle\alpha, \beta\rangle=$ $\langle L, R\rangle,\langle R, V\rangle,\langle V, L\rangle$ and the Majorana operators refer to those MBS at the trijunction as indicated in Fig. 8. We neglect in our considerations the Josephson coupling between the islands since it tends to increase all energy gaps. We will argue in the following that transfer of the MBS is adiabatic if it is carried out on a time scale

$$
T \gg \frac{\operatorname{arsinh}\left(E_{M}^{\max } / E_{M}^{\min }\right)}{E_{C}},
$$

in the parameter regime

$$
E_{C} \ll E_{M}^{\min } \lesssim E_{J, L / R}\left(\lesssim \frac{\left(E_{M}^{\min }\right)^{2}}{E_{C}}<\frac{\Delta^{2}}{E_{C}}\right) .
$$

Here, $E_{M}^{\min (\max )}$ denotes the minimal (maximal) value of

$$
E_{M}=\sqrt{E_{M, L R}^{2}+E_{M, R V}^{2}+E_{M, V L}^{2}}
$$

which is the energy of the fermionic mode created by fusing the three MBS at the trijunction.

Let us discuss the physics behind the different conditions in Eq. 688). First of all, the condition $E_{J, L / R} \gg E_{C}$ implies that the charging energies on both the left and right island are strongly suppressed and the MBS pairs $\left(\gamma_{1}, \gamma_{2}\right)$ and $\left(\gamma_{3}, \gamma_{4}\right)$ are decoupled to exponential accuracy. Second, since $E_{M}^{\min } \gg E_{C}$, the charging energy on the vertical island is also exponentially small in $E_{M} / E_{C}$ during the entire process (see App. G): The vertical island is then strongly coupled either to the left or the right island. Under this condition, the Josephson coupling $E_{J, V}$ to the bulk superconductor attached to the vertical island would not be necessary at all. Connecting the vertical island to the bulk may, however, be useful in experiments to suppress charging effects on the vertical island even further if $E_{M}$ cannot be made sufficiently large in practice.

The condition $E_{M}^{\min } \lesssim E_{J, L}, E_{J, R}$ implies that the lowest energy scale of the system is set by $\Delta E \sim \sqrt{E_{M} E_{C}}$, similar to the Majorana-tunneling dominated regime discussed in Sec. IV B The Josephson plasma frequencies of the islands $\sim \sqrt{E_{J} E_{C}}$ or the Majorana couplings $\sim E_{M}$ are much larger. We therefore conclude that the gap of the lowest excited state $\left|\psi_{1}\right\rangle$ to the ground-state manifold is at least on the order of $\sqrt{E_{M} E_{C}}$. Using the adiabaticity criterion (45), we can write down the condition

$$
T \gg \int_{C} \frac{\sum_{\langle\alpha, \beta\rangle}\left|d E_{M, \beta \alpha}(\boldsymbol{\lambda})\right|}{E_{M} E_{C}}
$$

where $C$ denotes the curve in parameter space given by the three Majorana couplings $\boldsymbol{\lambda}=$ $\left(E_{M, L R}, E_{M, R V}, E_{M, V L}\right)$. The integral on the right-hand side of Eq. 69) scales roughly as stated by Eq. (67), which depends logarithmically on $E_{M}^{\max } / E_{M}^{\min }$.

We emphasize that it is important that the junctions at the branching point are not closed at the same time (then $E_{M} \approx 0$ and the denominator in Eq. $(69)$ diverges). If the vertical island is connected to the bulk, this would result in the situation shown in Fig. 66(b): Then the number of zero-energy MBS would be increased from four to six, which doubles the ground-state degeneracy. Since relevant gaps become small, the evolution would not necessarily be adiabatic any more and uncontrolled rotations of the state may result.

We finally note that the transfer would in principle also work if the rightmost condition in Eq. (68), $E_{J, L / R} \lesssim\left(E_{M}^{\min }\right)^{2} / E_{C}$, was not be satisfied. We added it here because the entire protocol may involve more steps where this condition may be needed, for example, to initialize the system.

\section{SUMMARY AND CONCLUSIONS}

In this paper, we have analyzed the energy spectrum of two tunnel-coupled topological nanowire segments, which is a candidate setup for testing the topological nature of Majorana bound states.

We have computed the energy spectrum of this device at low energies numerically and identified four different operating regimes with several fine-structure subregimes as depicted in Fig. 2 2 (i) We find a charge-dominated 
regime $E_{C} \gg E_{M}, E_{J, \alpha}$ utilizable for initialization and readout of Majorana bound states. (ii) We identify a single-island regime for $E_{M} \gg E_{C}, E_{J, \alpha}$. (iii) We find a double-island regime for $E_{J, \alpha} \gg E_{M}, E_{C}$, where charging effects are strongly suppressed with $\varepsilon_{P, \alpha} \sim$ $\left(E_{C} E_{J, \alpha}^{3}\right)^{1 / 4} \exp \left(-\sqrt{8 E_{J, \alpha} / E_{C}}\right)$. (iv) Finally, one can enter a regime with four MBS near zero energy, a regime allowing for topologically protected Majorana manipulations.

Based on the low-energy spectrum, we have analyzed the time scales for preparation, manipulation, and readout of MBS in this setup. We discussed specifically each step of the fusion-rule test suggested in Ref. [55] and also basic operations for manipulating MBS in nanowire networks as needed for braiding. It turns out that the time scale is limited from below by $\Delta t \gg A / E_{C} \sim 0.1 \mathrm{~ns}$, where $A$ depends on logarithmic terms in $E_{M} / E_{C}$ and $E_{J, \alpha} / E_{C}$ and from above by $\Delta t \ll 1 / \varepsilon_{P, \alpha}, 1 / E_{M}^{\min }$, which is the minimal ground-state energy splitting. In addition, the resetting step of the fusion-rule protocol includes a charge-relaxation process, which is not included in the above criteria. Charge-relaxation times of semiconductor quantum dots are typically on the order of $10 \mathrm{~ns}$.

Since all the time-scale estimates we give are rather conservative, it would be interesting to see to which extent one could do better than the time-scale window given here. This would require a more accurate description of the Josephson energy for the nanowire junctions, both for the connection to the bulk superconductors and between the two islands. Since the charging energy might not be very small compared to the superconducting gap in the experimental devices [45, future studies should also include the effect of quasiparticles. Quasiparticle poisoning has been studied in particular for nontopological transmon qubits 74 77] and also aiming at topological devices 65, 78, 79, but especially the interplay with charging energy has, to our knowledge, not yet been explored.

\section{Acknowledgments}

We acknowledge stimulating discussions with David Aasen, Jason Alicea, Joshua A. Folk, Fabian Hassler, Andrew Higginbotham, Thomas S. Jespersen, Ferdinand Kuemmeth, Charles M. Marcus, and Ryan V. Mishmash. We acknowledge support from the Crafoord Foundation (M. L. and M. H.), the Swedish Research Council (M. L.), and The Danish National Research Foundation (K. F.).

\section{Appendix A: Derivation of the Hamiltonian}

In this Appendix, we derive the island Hamiltonian (6) and tunneling Hamiltonian (20) presented in Sec. II of the main part starting from a BCS description. Our effective low-energy Hamiltonian is valid at energies much smaller than the superconducting gap $(E \ll \Delta)$. Aside from this, we define the phase-basis states we introduced in Eqs. (13) and (14) in the main part and give the connection to the definition of the Majorana operators.

\section{Island Hamiltonian}

The island Hamiltonian $H_{I}=H_{F}+H_{C}$ is given by two contributions. The first, single-particle part $H_{F}$ describes the fermionic modes of the islands and takes the form of a BCS Hamiltonian:

$$
\begin{aligned}
H_{F}(\varphi)= & \int d^{3} x\left\{\sum_{\sigma} \hat{\psi}_{\sigma}^{\dagger}(x) H_{0}(x) \hat{\psi}_{\sigma}(x)\right. \\
& \left.+\left(\Delta(x) e^{-i \varphi} \hat{\psi}_{\uparrow}^{\dagger}(x) \hat{\psi}_{\downarrow}^{\dagger}(x)+\text { H.c. }\right)\right\} .
\end{aligned}
$$

Here, $\hat{\psi}_{\sigma}(x)$ denotes field operators for electrons with spin $\sigma$. The first part, $H_{0}(x)$, contains all effects not related to superconductivity and especially the spin-orbit coupling and Zeeman energy, which are needed to drive the island into a topologically nontrivial state. The second line of Eq. A1 incorporates the superconductivity in a BCS description. The superconducting gap is given by $\Delta(x)$, which is nonzero in the metal part of the island, and the superconducting phase of the island is given by $\varphi$.

The second contribution to the island Hamiltonian is the Coulomb interaction energy,

$$
H_{C}(\varphi)=E_{C}\left(\frac{2}{i} \frac{\partial}{\partial \varphi}+\hat{n}_{e}-n_{g}\right)^{2},
$$

which incorporates the total number of electrons, given by twice the number $N_{C}$ of Cooper pairs in the condensate $\left(2 \hat{N}_{C}=-2 i \partial / \partial \varphi\right)$ plus unpaired electrons occupying other fermionic modes:

$$
\hat{n}_{e}=\sum_{\sigma} \int d^{3} x \hat{\psi}_{\sigma}^{\dagger}(x) \hat{\psi}_{\sigma}(x) .
$$

The gating $n_{g}$ in Eq. A2 accounts for the effect of the applied gate voltages, which change the number of electrons on the island that minimizes the energy

The Hamiltonian is expressed here in the phase basis, $\left|\varphi, \mathbf{n}_{e}\right\rangle$, which characterizes the Cooper-pair condensate and $\left|\mathbf{n}_{e}\right\rangle=\left|n_{e, 0}, n_{e, 1}, \ldots\right\rangle$ contains the occupation numbers for modes of unpaired electrons. The phase-basis states can be expressed as

$$
\left|\varphi, \mathbf{n}_{e}\right\rangle=\frac{1}{\sqrt{2 \pi}} \sum_{N_{C}} e^{-i \varphi N_{C}}\left|N_{C}, \mathbf{n}_{e}\right\rangle,
$$

where $\left|N_{C}, \mathbf{n}_{e}\right\rangle$ is a state with a well-defined number of $N_{C}$ Cooper pairs. Since $N_{C}$ is an integer, the basis states are $2 \pi$-periodic in $\varphi,\left|\varphi+2 \pi, \mathbf{n}_{e}\right\rangle=\left|\varphi, \mathbf{n}_{e}\right\rangle$, and so are the wave functions in phase-space representation, $\psi_{\mathbf{n}_{e}}(\varphi)=$ $\left\langle\varphi, \mathbf{n}_{e} \mid \psi\right\rangle$. 
To proceed, we remove the superconducting phase from the single-particle part along the lines of, e.g., Ref. 80 by a unitary transformation of the Hamiltonian and the states,

$$
H^{\prime}=U H U^{\dagger}, \quad|\psi\rangle^{\prime}=U|\psi\rangle,
$$

choosing (different from Ref. 80 )

$$
U=e^{-i \varphi \hat{n}_{e} / 2} .
$$

The transformed Hamiltonians and states introduced in this Appendix are the ones used in the main part (where we leave out the prime for simplicity). Applying the unitary transformation to the single-particle part yields

$$
H_{F}^{\prime}=U H_{F}(\varphi) U^{\dagger}=H_{F}(0)=\sum_{i>0} E_{i} \hat{\chi}_{i}^{\dagger} \hat{\chi}_{i}
$$

where in the last step we diagonalized $H_{F}$ with eigenenergies $E_{i} \geq 0$ and Bogoliubov field operators

$$
\hat{\chi}_{i}=\sum_{\sigma} \int d x\left\{a_{i, \sigma}(x) \hat{\psi}_{\sigma}(x)+b_{i, \sigma}(x) \hat{\psi}_{\sigma}^{\dagger}(x)\right\} .
$$

When expressing $H_{F}^{\prime}$ in its eigenmodes, we assumed that the wire is infinitely long. The single-particle part thus possesses a mid-gap mode $i=0$ at energy $E_{0}=0$ with field operator $\hat{\chi}_{0}$, which does not appear in the sum of Eq. A7). Here and in the rest of this paper we refer to this mode as the 'zero-energy mode' or 'Majorana mode', even though this mode may move away from zero energy when adding the effect of the charging energy or when the wire has finite length. We next exclude all modes with finite energy $E_{i>0} \sim \Delta$, which means that we may simply drop $H_{F}^{\prime}$ when projecting on the low-energy subspace. We decompose the only remaining field operator $\hat{\chi}_{0}$ into the Majorana operators $\hat{\gamma}_{1}, \hat{\gamma}_{2}$ by

$$
\hat{\chi}_{0}=\hat{\gamma}_{1}+i \hat{\gamma}_{2} .
$$

Exploiting Eq. A8, the Majorana operators read

$$
\hat{\gamma}_{n}=\sum_{\sigma} \int d^{3} x\left\{c_{n i \sigma}(x) \hat{\psi}_{\sigma}(x)+c_{n \sigma i}^{*}(x) \hat{\psi}_{\sigma}^{\dagger}(x)\right\},
$$

where we chose the functions $c_{n i \sigma}(x)$ such that they are exponentially localized either at the left end (for $n=1$ ) or the right end (for $n=2$ ) of the wire.

We next transform the charging energy,

$$
H_{C}^{\prime}=U H_{C} U^{\dagger}=E_{C}\left(\frac{2}{i} \frac{\partial}{\partial \varphi}-n_{g}\right)^{2},
$$

in which the term from the unpaired fermionic modes has been removed. The information about the parity of the island is now contained in the boundary conditions of the wave function in phase space. The low-energy subspace is spanned by the basis states

$$
\left|\varphi, p_{12}\right\rangle^{\prime}=e^{-i \varphi p_{12} / 2}\left|\varphi, p_{12}\right\rangle
$$

where $p_{12}=0,1$ is the occupation number of the zeroenergy mode. We have left out here the reference to all other unpaired fermionic modes, which we assume for simplicity to be occupied by an even number of electrons 97. The phase basis states can now be written as in the main part, Eqs. 13) and (14):

$$
\begin{aligned}
\left|\varphi, p_{12}\right\rangle^{\prime} & =\frac{1}{\sqrt{2 \pi}} \sum_{N_{C}} e^{-i \varphi\left(2 N_{C}+p_{12}\right) / 2}\left|N_{C}, p_{12}\right\rangle(\mathrm{A} \\
& =\frac{1}{\sqrt{2 \pi}} \sum_{n \text { with parity } p_{12}} e^{-i \varphi n / 2}|n\rangle
\end{aligned}
$$

Moreover, physical wave functions have to obey paritydependent boundary conditions:

$$
\psi_{p_{12}}^{\prime}(\varphi+2 \pi)=(-1)^{p_{12}} \psi_{p_{12}}^{\prime}(\varphi) .
$$

The above boundary conditions are closely related to the phase-space representation of the number operator: One possibility is to use periodic boundary conditions (as before the unitary transformation), then $-2 i \partial / \partial \varphi$ counts the number of fermions in the Cooper-pair condensate. The other possibility is to use (anti)periodic boundary conditions and then $-2 i \partial / \partial \varphi$ counts the number of fermions contained in the Cooper-pair condensate plus the Majorana mode. This can be easily seen by comparing Eqs. (A4) and A13).

For an island without charging energy and a welldefined, fixed phase $\varphi$, the Hilbert space can be further reduced to that of a two-level system $\left|p_{12}\right\rangle^{\prime}=\left|\varphi, p_{12}\right\rangle^{\prime}$. In this case, the zero-mode field operator acts as

$$
\begin{aligned}
& \hat{\chi}_{0}\left|p_{12}=1\right\rangle^{\prime}=\left|p_{12}=0\right\rangle^{\prime}, \\
& \hat{\chi}_{0}\left|p_{12}=0\right\rangle^{\prime}=0,
\end{aligned}
$$

and using Eq. A9, the Majorana operators flip the parity:

$$
\begin{aligned}
\hat{\gamma}_{1}\left|p_{12}\right\rangle^{\prime} & =\left|\bar{p}_{12}\right\rangle^{\prime}, \\
\hat{\gamma}_{2}\left|p_{12}\right\rangle^{\prime} & =i^{2 p_{12}-1}\left|\bar{p}_{12}\right\rangle^{\prime} .
\end{aligned}
$$

Such a simplified description of the Hilbert space is reasonable when the tunneling energies in our setup are large compared to the charging energy.

When the charging energy is increased, phase fluctuations become important and phase is no longer a good quantum number. In this case, Eqs. A18 and A19 should only be understood as formal definitions of the Majorana operators and one has to be careful when trying to assign a physical meaning to them. Applying the Majorana operators to a general state $\psi_{p_{12}}^{\prime}(\varphi)$ that obeys the boundary condition A15, produces a new wave function that violates the periodicity condition and is thus not physical. Specifically, the Majorana operators interchange the wave function components in the even and odd parity sector. Thus, the odd (even) component of the new wave function obeys (anti)periodic boundary conditions, i.e., the opposite of what Eq. A15 requires. 
Therefore, any observable, including the Hamiltonian, can in general not contain 'lonesome' Majorana operators $\hat{\gamma}_{n}$. Rather, the Majorana operators always appear in combination with phase operators, such as in Eqs. 16 and 17 ,

$$
e^{ \pm i \hat{\varphi} / 2} \hat{\gamma}_{1}\left|\varphi, p_{12}\right\rangle^{\prime}=e^{ \pm i \varphi / 2}\left|\varphi, \bar{p}_{12}\right\rangle^{\prime}
$$

These operators, when applied to a general wave function $\psi_{p_{12}}^{\prime}(\varphi)$, produce a state that does obey the boundary condition A15,

$$
e^{ \pm i \hat{\varphi} / 2} \hat{\gamma}_{1} \psi_{p_{12}}^{\prime}(\varphi)=e^{ \pm i \varphi / 2} \psi_{\bar{p}_{12}}^{\prime}(\varphi)
$$

and therefore can have a physical meaning. In addition, the operators $e^{ \pm i \hat{\varphi} / 2} \hat{\gamma}_{1}$ also have a simple interpretation in the number basis:

$$
e^{ \pm i \hat{\varphi} / 2} \hat{\gamma}_{1}=\sum_{n}|n \pm 1\rangle\langle n|,
$$

it simply shifts the charge state by one. Corresponding relations hold for $\hat{\gamma}_{2}$ as stated in the main part in Eqs. 17) and 19.

\section{Tunneling Hamiltonian}

For the derivation of the Majorana-Josephson coupling we start from a standard bilinear coupling of the two wire segments:

$$
H_{T}=\int d^{3} x\left[t(x) \hat{\psi}_{L}(x) \hat{\psi}_{R}^{\dagger}(x)+\text { H.c. }\right] .
$$

Applying the above unitary transformation (A6), the tunneling Hamiltonian is transformed to

$$
H_{T}^{\prime}=\int d^{3} x\left[t(x) e^{-i\left(\hat{\varphi}_{L}-\hat{\varphi}_{R}\right) / 2} \hat{\psi}_{L}(x) \hat{\psi}_{R}^{\dagger}(x)+\text { H.c. }\right]
$$

To express this Hamiltonian in terms of the Majorana operators, we proceed similar to Refs. 80 82 and invert the relation (A8) (re-introducing the island index $\alpha$ ),

$$
\begin{aligned}
\hat{\psi}_{\alpha}(x) & =f_{\alpha}(x) \hat{\chi}_{\alpha 0}+f_{\alpha}^{*}(x) \hat{\chi}_{\alpha 0}^{\dagger}+\ldots \\
& =k_{\alpha}(x) \hat{\gamma}_{\alpha 1}+i l_{\alpha}(x) \hat{\gamma}_{\alpha 2}+\ldots
\end{aligned}
$$

where ... denotes the contributions from finite-energy modes, which we drop in the low-energy description. In the last step, we have introduced Majorana operators according to Eq. A9, where $\hat{\gamma}_{L 1}=\hat{\gamma}_{1}, \hat{\gamma}_{L 2}=\hat{\gamma}_{2}, \hat{\gamma}_{R 1}=\hat{\gamma}_{3}$, and $\hat{\gamma}_{R 2}=\hat{\gamma}_{4}$ consistent with our labeling in Fig. 1 used in the main part. When inserting Eq. A26 into the expression A24 for the tunneling Hamiltonian, we can simplify the expression by assuming that $t(x)$ is a function localized in the vicinity of the junction, i.e., $k_{L}(x) t(x), l_{R}(x) t(x) \approx 0$. We may therefore neglect $\hat{\gamma}_{1}$ and $\hat{\gamma}_{4}$ from the integral and find

$$
H_{T}^{\prime} \approx \frac{E_{M}}{2} e^{-i\left(\hat{\varphi}_{L}-\hat{\varphi}_{R}\right) / 2} i \hat{\gamma}_{2} \hat{\gamma}_{3}+\text { H.c. }
$$

with $E_{M}=2 \int d^{3} x t(x) l_{R}(x) k_{L}(x)$. Assuming finally $E_{M}$ to be real, we obtain the expression for for the Majorana-Josephson coupling in the main part. Inserting Eq. A20 and Eq. A22 into the tunneling Hamiltonian, we finally obtain an operational definition for the tunneling Hamiltonian both in the phase- and the number-basis representation as used in our numerical calculations.

\section{Appendix B: Parameter choices and estimations}

This Appendix is dedicated to discussing the parameter choices and relations that we employ in our study. We first argue in App. B 1 why we neglect phase differences between the two bulk superconductors connected to the nanowire. We then give in App. B 2 a heuristic derivation of how the Majorana coupling $E_{M}$ and the Josephson coupling $E_{J, C}$ of the junction connecting the two topological superconducting islands are related. In App. B3, we finally motivate our parameter choices for the time-scale estimations of the fusion-rule protocol (Sec. V) regarding also their experimental feasibility.

\section{Phase differences across the junction}

We have set the phase difference between the two bulk superconductors (in the following denoted by $\chi$ ) to zero in the Hamiltonian (7). However, in contrast to superconducting circuits with a loop, the phase differences cannot be adjusted by an external magnetic flux for the device we consider. In this Appendix, we explain why this is nevertheless a permissible simplification in our analysis of the fusion-rule protocol.

First, phase differences between the bulk superconductors matter only if all three valves are at least partially open (step 1 and 2 of the fusion-rule protocol). Otherwise the disconnected parts of the semiconductor wire structure can be treated independently and a nonzero phase difference $\chi$ can simply be absorbed into a redefinition of the phases. This is not possible if all junctions are partially open. However, we have explicitly verified from our numerical simulations that the ground state of the system is assumed for zero phase difference $\chi=0$ irrespective of the values of all parameter values. Since the system adiabatically follows the ground state in step 1 and 2 , there is no reason why a nonzero offset phase should develop.

Second, in addition to such an offset phase, one should also investigate the effect of phase fluctuations, which are associated with current fluctuations. While these fluctuations do not affect the measurement outcomes for the charge sensing, they can affect the pumping current. For small phase differences $\chi$, one can write the energy of the $\chi$-dependent part of the energy as $E \sim E_{\min } \chi^{2} / 2$, where $E_{\text {min }}$ is roughly given by the smallest of the Majorana 
/ Josephson couplings $E_{\min }=\min \left(E_{J, \alpha}(t), E_{M}(t)\right)$ [98]. We neglect in our considerations the effect of a nonzero charging energy. Fluctuations in $\chi(t)$ cause a fluctuating current $I(t)=\partial E / \partial \chi(t)=E_{\min } \chi$ and the number of electrons to the left and right of the junction therefore fluctuates. This does not affect the measurement outcomes on average because $\chi$ fluctuates symmetrically around zero.

However, the fluctuations can affect the current noise, which means that one should repeat the cycle sufficiently many times to suppress noise effects. One may wonder whether the number of cycles needed to obtain an acceptable signal-to-noise ratio would not lead to measurement times that are long compared to, e.g., quasiparticle poisoning times. To this end, we estimate the number $\Delta N_{\text {cycle }}$ of electrons that are transferred in a typical cycle due to a fluctuating phase $\chi(t)$ (in addition to the pumped charge). For simplicity, we assume that $\chi$ is constant for each cycle and changes from one cycle to the other randomly. The typical number for $\Delta N_{\text {cycle }}$ thus reads $\Delta N_{\text {cycle }}=I \Delta t=E_{\min } \sqrt{\left\langle\chi^{2}\right\rangle} \Delta t$ where $\Delta t$ is the time duration of step 1 and 2 of the fusion-rule protocol. Treating $\chi$ as a classical variable, the average energy $E \sim E_{\min }\left\langle\chi^{2}\right\rangle / 2$ for the phase degree of freedom is given by half the temperature, $T / 2$, according to the equipartition theorem in thermal equilibrium. It follows

$$
\Delta N_{\text {cycle }}=\sqrt{E_{\min } T} \Delta t .
$$

To estimate $\Delta N_{\text {cycle, }}$, we insert in the above expression $E_{\min }(t)=E_{M}^{\max }=10 E_{C}$ and $\Delta t \sim 10 / E_{C}$ and $T=$ $E_{C} / 10$ from our considerations in the main part, which yields

$$
\Delta N_{\text {cycle }} \lesssim 10
$$

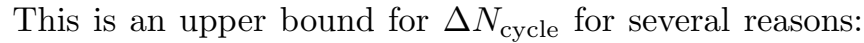
(i) The adiabaticity condition derived in the main part used for the value of $\Delta t$ has been estimated conservatively. (ii) The Majorana coupling $E_{M}^{\max }$ is rather smaller than $10 E_{C}$ and, in addition, $E_{\min } \leq E_{M}^{\max }$ during the cycle. (iii) The presence of a charging energy suppresses charge fluctuations. (iv) The assumption of a constant phase during each cycle leads to a maximal value for $\Delta N_{\text {cycle. }}$ If the correlation time of the phase fluctuations is smaller, i.e., phase fluctuations are much faster than the cycle period, the current fluctuations would average out. This means that the relevant $\Delta t$ would be much shorter than the time needed for the steps in the protocol. The fluctuating charge $\Delta N_{\text {cycle }}$ should thus be rather smaller than the above estimate. Since any charge-pumping experiment will be carried out with a large number of cycles in practice, we thus expect these experiments to have an acceptable signal-to-noise ratio.

\section{Relation between $E_{M}$ and $E_{J, C}$}

The Majorana tunneling energy $E_{M}$ and the Josephson energy $E_{J, C}$ cannot be controlled individually since both are tuned through the gate near the central valve. We here give a crude estimate of how they are related. The Josephson energy $E_{J, C}$ can be connected with the junction properties through the Ambegaokar-Baratoff formula [83]: $I_{c} R_{n}=\pi \Delta / 2 e$. Here, $I_{c}=2 \pi E_{J, C} / \Phi_{0}$ is the critical supercurrent through the junction with flux quantum $\Phi_{0}=h / 2 e$. Furthermore, $R_{n}$ denotes the normal resistance of the junction with $1 / R_{n} \sim\left(e^{2} / \hbar\right) \pi|t|^{2} \nu_{L} \nu_{R}$ with tunneling amplitudes $t$ and densities of states $\nu_{L / R}$ of the left and right wire segments, respectively. This yields the relation

$$
E_{J, C}=\frac{\pi^{2}}{4} \Delta|t|^{2} \nu_{L} \nu_{R}
$$

Note that the tunneling amplitude $t$ in this expression is related to the overlap of the extended bulk wave functions. The Majorana-Josephson energy $E_{M}$ is also a tunneling amplitude but it depends on the overlap of the Majorana bound states localized at the junction [84]. For simplicity, we take $E_{M} / t \sim\left\langle\psi_{L}^{\mathrm{maj}} \mid \psi_{R}^{\mathrm{maj}}\right\rangle /\left\langle\psi_{L} \mid \psi_{R}\right\rangle \sim L / \xi$. Here, we used that the bulk wave functions $\psi_{L / R}(x) \sim$ $1 / \sqrt{L}$ scale with the length $L$ of the segments, while the Majorana wave functions $\psi_{L / R}^{\text {maj }}(x) \sim 1 / \sqrt{\xi}$ rather scale with the coherence length $\xi$ of the superconducting islands. To estimate the density of states, we model the wire segments as effectively free one-dimensional electron gases, which yields $\nu_{L / R}=\sqrt{2 m^{*} L^{2} / E_{F}}$ with effective mass $m^{*}$ and Fermi energy $E_{F}$. Inserting these relations into Eq. B3, we obtain

$$
E_{J, C}=\frac{\pi^{2}}{4} \Delta \frac{2 m^{*} \xi^{2}}{E_{F}} E_{M}^{2} .
$$

Note that this relation is independent of the wire length as expected for a property of the junction. In the topological regime, the Fermi energy lies in the gap and can be estimated as $E_{F} \sim m^{*} \alpha^{2}[3]$ with the effective spinorbit velocity $\alpha$. Furthermore, in the strong spin-orbit regime, we can estimate $\xi \sim \alpha / \Delta$ as the ratio of the Fermi velocity and the gap (see Ref. 85] and supplemental material), which gives the simple result

$$
E_{J, C}=k \frac{E_{M}^{2}}{\Delta}
$$

where $k$ is a constant of close to 1 ; for the above values we obtain $k \approx 5$, which we use for our numerical results.

\section{Parameters choices and neglect of quasiparticle excitations}

Here we discuss the feasibility of the parameter ratios used in our time-scale estimations of the fusion-rule protocol and the braiding steps. Since our model does not include quasiparticle excitations, it is applicable only as long as the Majorana coupling satisfies $E_{C}, E_{M}^{\max } \lesssim \Delta$. Here, $\Delta$ is the superconducting gap, which we assume to 
be the same in the nanowire as in the bulk superconductor. This is not unrealistic in view of recent experiments [45, 65) measuring $\Delta$ on the order of a few K (e.g. for aluminum). The charging energy should thus be a few hundreds of $\mathrm{mK}$. While charging energies in prototypical nanowire structures are on the same order as $\Delta$ for wires of $\sim 1-2 \mu \mathrm{m}$ length [45, lower charging energies could be achieved by using longer nanowires. This would also be advantageous for suppressing the overlap of the Majorana bound states within each wire segment. Alternatively, the ratio $E_{C} / \Delta$ could be reduced by using niobium as a proximal superconductor instead, which has a larger gap reaching $8 \mathrm{~K}[86$. To initialize the MBS, it is furthermore important that $E_{C}, E_{M}^{\max } \gg T$, the thermal energy of the nanowire. This seems possible for fridge temperatures $\sim 20 \mathrm{mK}$ 45. Furthermore, one can control the maximal Majorana energy $E_{M}^{\max }$ by the gates and keeping it between $T$ and $\Delta$ should be no problem.

For our numerical results, we employ a large value of $\Delta / E_{C}=100$ since we assume $E_{C} \ll E_{M}^{\max } \ll \Delta$ in our analysis. This allows us to set $E_{M}^{\max } / E_{C}=10$ in our numerical calculations. In practice, $E_{M}^{\max } \sim E_{C}$ should be sufficient (replacing $\log \left(E_{M}^{\max } / E_{C}\right) \rightarrow 1$ in the timescale estimates) and smaller ratios $\Delta / E_{C}$ should thus work as well. Moreover, only the $E_{J, C}$ depends directly on $\Delta$ in our analysis and as long as $E_{M}^{\max } \ll \Delta, E_{J, C}$ is a rather small correction. We explicitly checked from our numerical analysis that a smaller ratio $\Delta=10 E_{C}$ does not lead to a suppression of the energy gaps in the spectrum.

The Josephson couplings to the bulk superconductors, $E_{J, \alpha}$, depend of course also on $\Delta$. However, the only important aspect is here that $E_{J, \alpha} / E_{C}$ is large enough to achieve a good suppression of charging effects. At least $E_{J, \alpha}^{\max } / E_{C}>10$ is needed [at $E_{J, \alpha}^{\max } / E_{C}=10$, one obtains $\varepsilon_{P} / E_{C} \sim 0.01$ from Eq. [37]]. We take $E_{J, \alpha}^{\max } / E_{C} \sim 50$ in our numerical calculations to make sure that $E_{J}^{\max } \gg$ $E_{M}^{\max }$. We note that the energy splittings arising from the Josephson energies scale as $\sim \sqrt{8 E_{J, C} E_{C}}, \sqrt{8 E_{J, \alpha} E_{C}}$, which does not exceed $\Delta$ for the values we assume. It is therefore reasonable to exclude quasiparticle excitations from our numerical calculations.

\section{Appendix C: Numerical diagonalization}

In this appendix, we discuss the advantages of numerically diagonalizing the Hamiltonian in the number basis as compared to the phase basis.

The number basis has a natural advantage because it is a countable basis due to the discreteness of the electron charge. By contrast, the phase basis is generated by a continuous variable, which has to be discretized in a numerical procedure. One then maps the Hamiltonian on a lattice model (see caption of Fig. 9 and Ref. [87). Taking a uniform mesh in phase space, we compare in Fig.
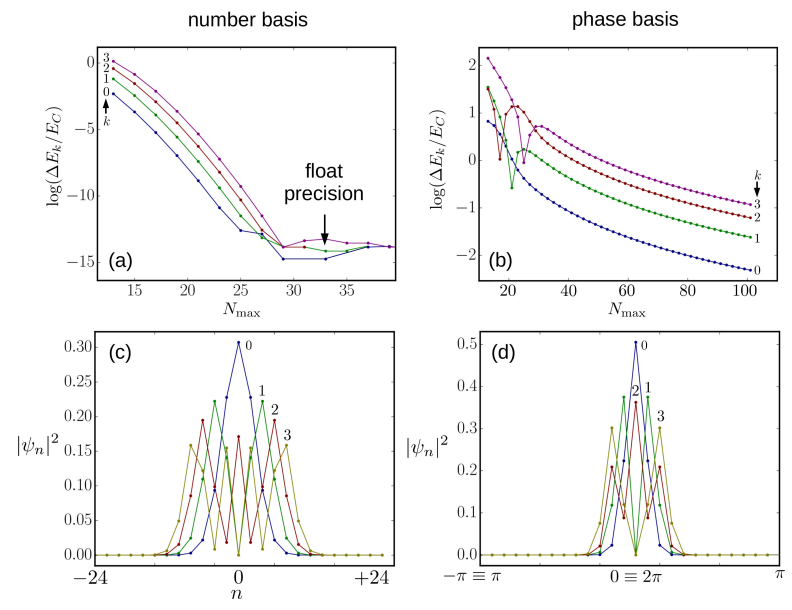

FIG. 9: Comparison of the convergence of the numerical approach in number space and phase space. (a) and (b) Energy difference of the four lowest eigenenergies $\Delta E_{k}\left(N_{\max }\right)=$ $E_{k}\left(N=N_{\max }\right)-E_{k}\left(N=N_{\max }-2\right)$ as a function of $N$ included basis states. The states are labeled in ascending order $(k=0,1,2,3)$. We show the result both in number space (a) and in discretized phase space (b). The eigenenergies are obtained from a single-island Hamiltonian, i.e., $H=E_{C}\left(\hat{n}-n_{g}\right)^{2}+E_{J}(1-\cos (\hat{\varphi}))$, see Eq. (5). In (c) and $(\mathrm{d})$, we show the corresponding probability densities in number and discretized phase space for the case $N_{\max }=25$. Note that we only include even electron numbers (i.e., the points in (c) differ by $\Delta n=2$ ). To compute the eigenenergies and eigenfunctions in phase space, we express the corresponding Schrödinger equation in phase space with the replacement $\hat{n}^{2} \rightarrow-\partial^{2} / \partial \varphi^{2}$ and discretize the phase variable, $\psi(\varphi) \rightarrow \psi\left(\varphi_{j}\right), j=1, \ldots, N, \varphi_{j}=j \cdot 2 \pi / N_{\max }$ and the second derivative $\partial^{2} \psi(\varphi) / \partial \varphi^{2}=\psi\left[\left(\varphi_{j+1}\right)+\psi\left(\varphi_{j-1}\right)-\right.$ $\left.2 \psi\left(\varphi_{j}\right)\right] /\left(2 \pi / N_{\max }\right)^{2}$. For the even-parity sector, we impose periodic boundary conditions, i.e., $\psi\left(\varphi_{N_{\max }+1}\right)=\psi\left(\varphi_{1}\right)$. The results are shown for $E_{J} / E_{C}=100$ and $n_{g}=0$.

9 the convergence of the lowest energy eigenstates of a single-island Hamiltonian when increasing the number of basis states both in number and in phase basis. We can clearly see that the number-basis approach converges exponentially fast, whereas the phase-basis approach yields only algebraic convergence.

The reason is that the wave function in phase space is sharply localized near $\varphi=0$ and therefore one needs a very fine resolution to describe the wave function properly. When taking $N_{\max }=25$ for the parameters used in Fig. 9(d), the wave function is localized around a few points around $\varphi=0$, which is equivalent to $\varphi=2 \pi$. To improve the performance in phase space, one could use a nonuniform mesh for the discretization. However, a grid with a fine resolution around zero phase would only work optimal for energetically low-lying states, so it might be useful to adapt the grid for different parts of the spectrum.

One may of course argue that limiting the maximally included number state to $|n| \leqslant N_{\max }$ also introduces an approximation similar to using a finite number of points 
in phase space. However, as we can see in Fig. 9 (c), the probabilities for different number states are still localized only to a number of $n \lesssim 10$ states and they drop exponentially for $n \gtrsim 10$. This is in accordance with our estimate $N_{\max } \sim \sqrt{E_{J} / E_{C}} \sim 10$ given in Sec. III for $E_{J} / E_{C}=100$ as used in Fig. 9. Moreover, the accuracy for energetically higher-lying states can be improved by including more charge states depending on its energy $E / E_{C}$. Thus, choosing a finite set of states for the numerical diagonalization is much easier in the number basis as compared to the phase basis.

Another advantage of the number basis approach is that the interpretation of the wave functions is simpler. We are also interested in the regime when the charging energy is the dominant energy scale (discussed in Sec. IV A which is useful for initialization and readout of parity states. In this regime, the wave function is not localized in phase space but in charge space. Also, numberspace probability distributions are directly related to the measurement outcomes. All these points are different from the situations studied in other works [42, 53], where charging effects are always one of the smallest energy scales. In these cases, it may be advantageous to use the phase basis.

\section{Appendix D: Effect of central Josephson coupling and asymmetries of bulk Josephson couplings}

In our discussion of the parameter regimes of the coupled topological Cooper pair boxes in Sec. [V] of the main part, we assumed (i) zero central Josephson coupling, $E_{J, C}=0$ and (ii) symmetric Josephson couplings to the bulk superconductors, $E_{J, L}=E_{J, R}$. We next extend this analysis by investigating the effects of loosening each of the assumptions separately.

\section{Effect of central Josephson coupling}

We first investigate the effect on the low-energy spectrum of including the central Josephson coupling $E_{J, C}$. Any tunnel coupling leading to a nonzero $E_{M}$ will also involve a nonzero $E_{J, C}$, i.e., these Josephson couplings cannot be switched off individually. Our crude estimate in App. B 2 yielded the relation $E_{J, C} \sim E_{M}^{2} / \Delta$, that is, the ratio $E_{J, C} / E_{M}$ is tunable with $E_{M}$. Our analysis here shows that the relevant energy splittings increase when $E_{J, C}$ is included (see Fig. 10). This implies that the time scale estimates given in the main part are conservative since they assume the "worst-case scenario" of $E_{J, C}=0$.

For simplicity, we focus our discussion here on zero bulk Josephson couplings, $E_{J, L}=E_{J, R}=0$, and sym-

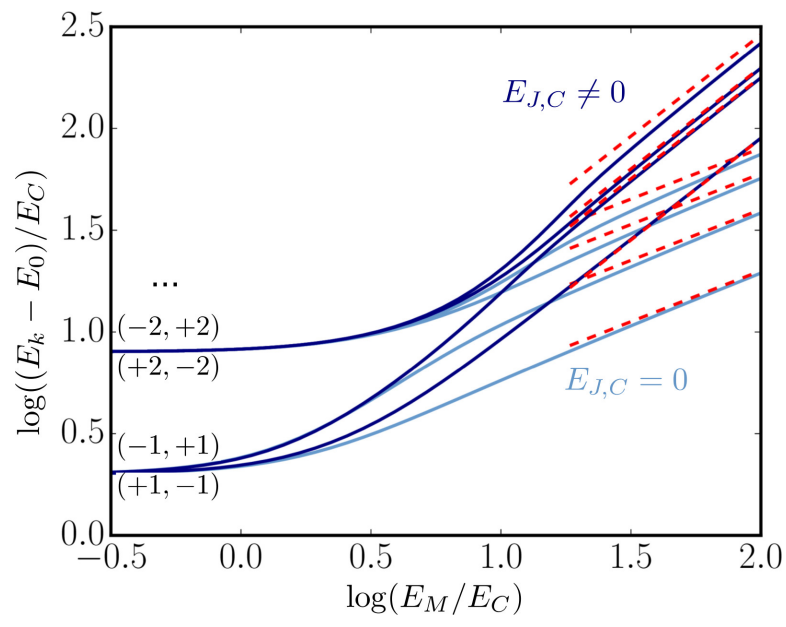

FIG. 10: Effect of the conventional Josephson coupling of the central junction $\left(E_{J, C}\right)$ on the low-energy spectrum. Solid lines show the numerically computed energy splitting between the first four excited states with zero excess charge, $N=0$, (at energies $E_{k}$ ) and the ground state (at energy $E_{0}$ ) as a function of the Majorana-Josephson coupling $E_{M}$. The center Josephson coupling is given by $E_{J, C}=5 \cdot E_{M}^{2} / \Delta$, which we exclude $\left(E_{J, C}=0\right)$ and include $\left(E_{J, C} \neq 0\right)$ from the computation as indicated. The red-dashed lines indicate the approximations for the splittings from Eq. (D2) for $E_{J, C}=0$ and from Eq. (D5) for $E_{J, C} \neq 0$. All the states are continuously connected to charge states $\left(n_{L}, n_{R}\right)$ for $E_{M}=0$ as indicated at curves on the left. The other parameters are $E_{C, L}=E_{C, R}=E_{C}$, $E_{J, L}=E_{J, R}=0, \Delta=100 E_{C}$, and $n_{g, L}=n_{g, R}=0$.

metric charging energies, $E_{C, L}=E_{C, R}=E_{C}$. Using the notation of Sec. IID the Hamiltonian 27) reads

$$
\begin{aligned}
H= & 2 E_{C}\left[\left(\frac{\hat{N}}{2}\right)^{2}+\left(\frac{\Delta \hat{n}}{2}\right)^{2}\right] \\
& +E_{M} \cos \left(\frac{\Delta \hat{\varphi}}{2}\right) i \hat{\gamma}_{2} \hat{\gamma}_{3}+E_{J, C}(1-\cos (\Delta \hat{\varphi}))
\end{aligned}
$$

As discussed in Sec. IV B the total number of electrons is conserved and it is sufficient to focus only on the $N=0$ subspace. Equation (D1) is similar to the Hamiltonian of a single Cooper pair box with the only difference that the phase-dependent term includes two harmonics. We compare the resulting $E_{M}$-dependence of the low-energy spectrum in Fig. 10 for $E_{J, C}=0$ and $E_{J, C}=5 E_{M}^{2} / \Delta$ as indicated. The spectrum for $E_{J, C}=0$ has been analyzed in Sec. IVB and for $E_{M} \gg E_{C}$, the energy levels are well-approximated by

$$
E_{k} \approx \sqrt{4 E_{C} E_{M}}(k+1 / 2) .
$$

The simple physical picture is here that the Majorana term couples adjacent charge states of the islands $\left(n_{L}, n_{R}\right) \leftrightarrow\left(n_{L} \pm 1, n_{R} \mp 1\right)$, which leads to single-electron plasma oscillations.

This changes when the Josephson coupling becomes nonneglible for $E_{J, C} \gtrsim E_{M}$. Let us derive an approximation of the eigenenergies in the limit $E_{J, C} / E_{M} \gg 1$ 

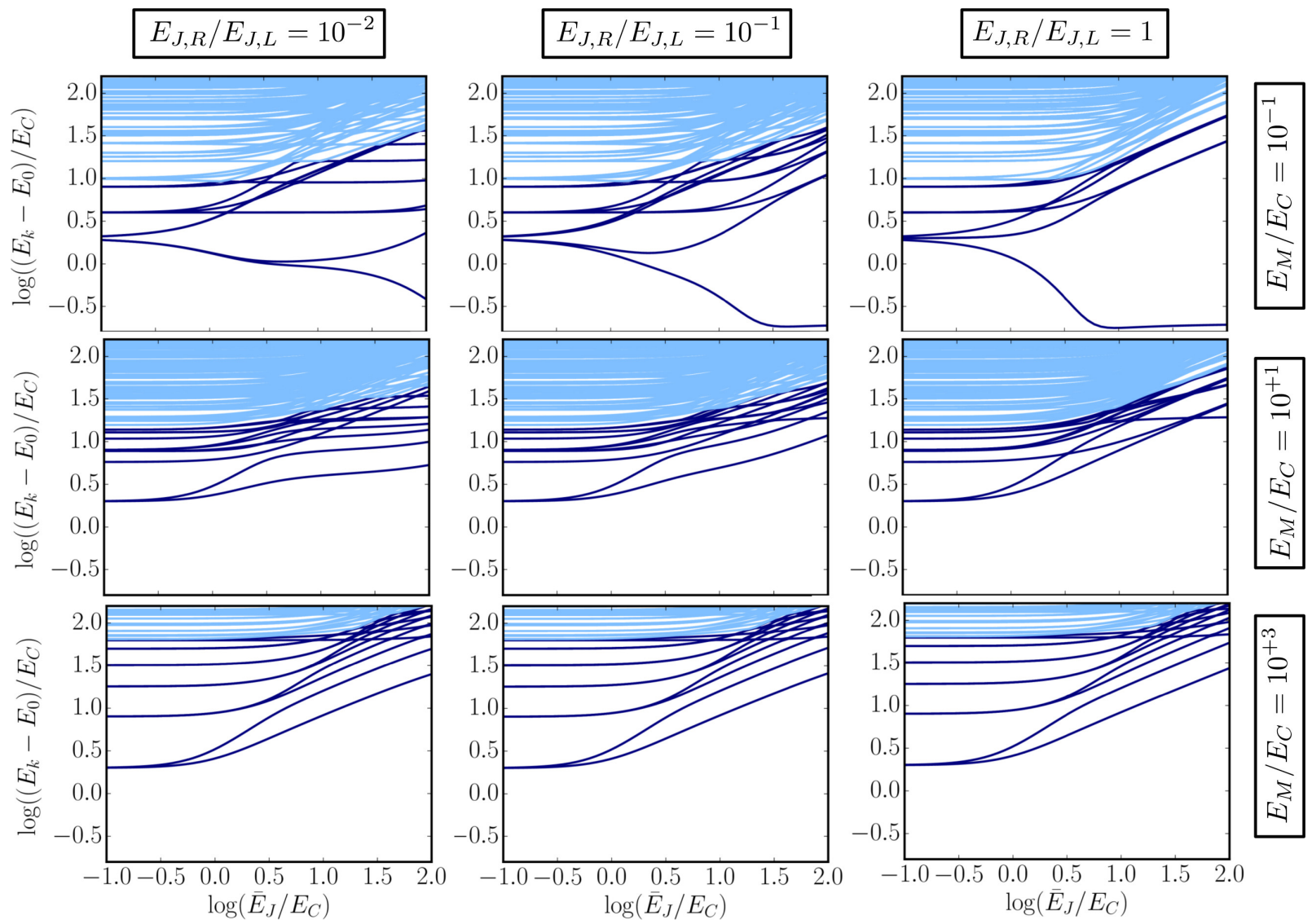

FIG. 11: Low-energy spectrum of two hybridized topological superconducting islands asymmetrically coupled to bulk superconductors. The figure shows the numerically computed energy splittings between succeeding excited states (at energy $E_{k}$ ) and the ground state (at energy $\left.E_{0}\right)$ as a function of the average Josephson coupling $\bar{E}_{J}=\left(E_{J, L}+E_{J, R}\right) / 2$ to the bulk superconductors. We highlight the 12 lowest states in darker color for comparison with Fig. 3 and show all higher-lying states in brighter color. The Majorana coupling $E_{M}$ is changed from one row to the other while the asymmetry $E_{J, R} / E_{J, L}$ of the Josephson couplings is changed from one column to the other as indicated. We use $n_{g, L}=n_{g, R}=0, E_{J, C}=0$, and $N_{\max }=20$.

to contrast it with the physics of the case of $E_{J, C}=0$. In the former case, the system behaves as two copies of Cooper pair-plasma oscillators corresponding to the two possible parity degrees of freedom of the island (eveneven and odd-odd parity; note that we restrict our considerations to even total parity). The ordinary Cooper pair tunneling across the junction can only couple states within the even-even or odd-odd parity sector, changing the number of electrons on both islands by two. Neglecting the fractional Josephson term $\propto \cos (\Delta \hat{\varphi} / 2)$, the eigenstates are given by the degenerate Josephson plasmon states $\left|k, 0_{12} 0_{34}\right\rangle$ and $\left|k, 1_{12} 1_{34}\right\rangle$ with energies $E_{k}=\sqrt{8 E_{J, C} E_{C}}(k+1 / 2)$.

The two parity states then become mixed due to the fractional Josephson term $\propto \cos (\Delta \hat{\varphi} / 2)$, yielding bonding and antibonding combinations for the eigenstates 99]:

$$
\begin{aligned}
\left|k, 0_{23} 0_{14}\right\rangle & =\frac{1}{\sqrt{2}}\left[\left|k, 0_{12} 0_{34}\right\rangle+\left|k, 1_{12} 1_{34}\right\rangle\right], \\
\left|k, 1_{23} 1_{14}\right\rangle & =\frac{1}{\sqrt{2}}\left[\left|k, 0_{12} 0_{34}\right\rangle-\left|k, 1_{12} 1_{34}\right\rangle\right] .
\end{aligned}
$$

This corresponds to fusing the MBS pair $\left(\gamma_{2}, \gamma_{3}\right)$ localized at the central junction. The eigenenergies are split according to the nonlocal parity $\left\langle i \gamma_{2} \gamma_{3}\right\rangle= \pm 1$, yielding

$$
E_{k, \pm}=\sqrt{8 E_{J, C} E_{C}}(k+1 / 2) \mp E_{M},
$$

where the minus (plus) sign corresponds to the (anti)symmetric combination, respectively. The associated energy gaps to the ground state with energy $E_{0,+}$ are shown as red-dashed lined in Fig. 10. Provided the charging energy is known, one could actually determine both $E_{J, C}$ and $E_{M}$ independently from the low-energy spectrum in an easy way using Eq. (D5).

This shows that the physics and also the energy spectrum is different depending on whether the Josephson Cooper pair coupling or the single-electron tunneling dominates. 


\section{Asymmetric Josephson couplings to bulk superconductors}

In this Appendix, we turn to the more general situation when the Josephson energies with the two bulk superconductors are asymmetric $\left(E_{J, L} \neq E_{J, R}\right)$. We show that the importance of asymmetries is suppressed the larger the Majorana coupling $E_{M}$ becomes.

The effect of asymmetric Josephson energies on the low-energy spectrum is illustrated in Fig. 11. We change the ratio $E_{J, R} / E_{J, L}$ in the horizontal direction and $E_{M}$ in the vertical direction. The figure shows that asymmetries have a large effect on the spectrum when the coupling $E_{M}$ between the islands is small $\left(E_{M} / \bar{E}_{J} \ll 1\right.$ with $\bar{E}_{J}=$ $\left(E_{J, L}+E_{J, R}\right) / 2$, upper row), whereas the effect is rather small if the coupling $E_{M}$ is large $\left(E_{M} / \bar{E}_{J} \gg 1\right.$, lower row). This finding can be readily understood from the representation (27) in sum and difference variables. It takes the form

$$
H=\tilde{H}+\left(E_{J, L}-E_{J, R}\right) \sin \left(\frac{\hat{\Phi}}{2}\right) \sin \left(\frac{\Delta \hat{\varphi}}{2}\right)
$$

with a part $\tilde{H}$ that is independent of the asymmetry, that is, it depends only on the average $\bar{E}_{J}$. As soon as the phase dynamics are locked, $\Delta \varphi=\varphi_{L}-\varphi_{R} \approx 0$ for $E_{M} \gg \bar{E}_{J}$, the asymmetry-dependent term can be dropped. The simple physical picture is that the system behaves as a single superconducting island connected to the bulk superconductors by two Josephson junctions in parallel (with zero flux enclosed).

This indicates that charging effects can be efficiently suppressed on both segments even if only one of them is coupled to a bulk superconductor. This finding is relevant for transferring MBS in nanowire networks as we discuss in Sec. VI A 2. Interestingly, it turns out that the requirement $E_{M} \gg E_{J}$ is, in fact, not even needed for shuffling MBS between the segments as we show in Sec. VIA2

\section{Appendix E: Time-scale conditions for readout}

Sections [V] and VI only focused so far on the time scales required for preparing and manipulating MBS in nanowire structures. In this Appendix, we complement these considerations by discussing the time scales for performing the readout of the MBS.

\section{Charge sensing}

By tuning the islands to the charge-dominated regime different parity states of the island are mapped onto different charge states. The charge state is then read out by simple charge sensors well-known from quantum-dot physics. This technique works as long as the readout time, $\tau_{D} \ll 1 / E_{M}^{\min }, 1 / E_{J, \alpha}^{\min }$, is much shorter than all time scales related to tunneling of electrons or Cooper pairs, respectively. As discussed in Ref. [55, one expects $\tau_{D}<1 \mu$ s with standard charge readout techniques.

\section{Charge-pumping readout}

An alternative readout method is to pump charge conditioned upon the fermion parities of the islands. The steps of the pumping procedure, explained in detail in Ref. [55], are sketched in Fig. 12(a). Before we derive time-scale conditions for the pumping procedure, we briefly review the steps.

Parity-dependent charge pumping. The simplest way to understand this pumping procedure is to follow the steps sketched in Fig. 12(a). In step 1, one reduces the Josephson energies $E_{J, \alpha}$ from $E_{J, \alpha}^{\max }$ after step 2 of the fusion-rule protocol (see Fig. 4) until they reach an energy $E_{J, \alpha}^{\mathrm{sw}}$ on the order of the charging energy $E_{C}$. In fact, reducing $E_{J, \alpha}$ is not needed at all as we explain at the end of this section but it is helpful for the illustration of the mechanism. We assume that the gate voltages are adjusted such that the lowest state of even-even parity is $\left|n_{L}, n_{R}\right\rangle=|0,0\rangle$, while the lowest state of odd-odd parity is given by $|-1,+1\rangle$. As indicated in Fig. 12(b), one then in step 2 adiabatically sweeps the gate voltages from $n_{g, \alpha}^{(1)} \rightarrow n_{g, \alpha}^{(2)}$. This is done such that one crosses a charge-degeneracy point for odd-odd parity [mapping $|-1,+1\rangle \rightarrow|+1,-1\rangle]$ but not for even-even parity [leaving $|0,0\rangle$ unchanged]. Consequently, a Cooper pair is transferred between the islands and the bulk superconductors dependent on the island parities. To complete the pumping, one closes the junctions to the bulk superconductors (step 3 ), $E_{J, \alpha} \rightarrow E_{J, \alpha}^{\min }$, opens the central valve $E_{M} \rightarrow E_{M}^{\max }$ (step 4 ), and sweeps the gate voltages back to their original values: $n_{g, \alpha}^{(2)} \rightarrow n_{g, \alpha}^{(1)}$ (step 5). Finally, one lets the system relax to the unique ground state again.

Proceeding in this way, one expects for the fusion-rule experiment discussed in Sec. V a pumping current of $I=1 / 2 \times 2 e \times f$, where $f$ is the pumping frequency. The factor $1 / 2$ accounts for the $50 \%$ probability to project the prepared state $\left(\left|0_{12} 0_{34}\right\rangle+\left|1_{12} 1_{34}\right\rangle\right) / \sqrt{2}$ on the odd-odd parity combination. This contrasts with the nontopological case: If there are no subgap states close to zero energy, the islands always remain in the even-even parity configuration and no charge is pumped. In the presence of notopological subgap states, it is further unlikely that the odd-odd combination appears with $50 \%$ probability because these states are unlikely to be at exactly at zero energy. The probability should also depend sensitively on the parameters. We emphasize again that we assumed in our discussion that the total parity of the islands does not change even over many cycles.

We now come back to our initial comment that it is in fact not needed to lower the Josephson energies down to $E_{J, \alpha} \sim E_{C}$ in step 1 . The gate-voltage sweep 
in step 2 could be carried out for any $E_{J, \alpha}^{\mathrm{sw}}$ as long as $E_{J, \alpha}^{\mathrm{sw}} \gtrsim E_{C}$. The only important aspect is that the gate voltage is swept over a point that would become a charge-degeneracy point for $E_{J, \alpha}=0$ for the oddodd parity subspace $\left[n_{g, \alpha}=0\right.$ in Fig. 12(b)]. By contrast, one must not cross over points that would become charge-degenerate for $E_{J, \alpha}=0$ for the even-even subspace $\left[n_{g, \alpha}= \pm 1\right.$ in Fig. 12(b)]. The projection on a particular charge state happens when closing the outer valves $\left(E_{J, \alpha} \rightarrow E_{J, \alpha}^{\min }\right)$. The time-scale estimation in the next paragraph shows that it is even favorable to perform the gate-voltage sweep at $E_{J, \alpha}^{\mathrm{sw}}=E_{J, \alpha}^{\max }$.

Time-scale condition. We next show that this charge pumping can proceed adiabatically if the gate voltage sweep in step 2 is performed on a time scale

$$
T_{2} \gg \frac{1}{E_{J, \alpha}^{\mathrm{sw}}} \quad\left(E_{J, \alpha}^{\mathrm{sw}} \gtrsim E_{C}\right) .
$$

It is thus most favorable to perform the gate sweep at $E_{J, \alpha}^{\mathrm{sw}}=E_{J, \alpha}^{\max }$. The manipulations of the Josephson and Majorana tunneling energies have to be performed on time scales $T_{i} \gg 1 / E_{C}$ as worked out earlier in the main part.

To verify Eq. E1), we again start from the adiabaticity condition 45 . Ideally, the gate voltages should be manipulated such that the Josephson and Majorana energies are kept constant and only the charging energy is manipulated through the gatings $n_{g, \alpha}$. In this case, we can express Eq. 45 as

$$
T_{2} \gg \int_{n_{g, \alpha}^{(1)}}^{n_{g, \alpha}^{(2)}} d n_{g, \alpha}\left|\frac{\left\langle\psi_{k}\left|\partial H / \partial n_{g, \alpha}\right| \psi_{0}\right\rangle}{\left(E_{k}-E_{0}\right)^{2}}\right| .
$$

We check the above condition for the lowest excited state $(k=1)$. Using $\left|\left\langle\psi_{1}(t)\left|\partial H(t) / \partial n_{g, \alpha}\right| \psi_{0}(t)\right\rangle\right| \sim E_{C}, n_{g, \alpha}^{(2)}-$ $n_{g, \alpha}^{(1)} \sim 1$, and estimating the minimal gap as $E_{1}-E_{0} \geqslant$ $\sqrt{E_{J, \alpha}^{\mathrm{sw}} E_{C}}[100$, one obtains Eq. E1.

The steps of changing the Majorana coupling $E_{M}$ and the Josephson coupling $E_{J, \alpha}$ (steps 1, 3, and 4) have to be performed on the same time scales given by Eqs. (1) and (2) as derived in Sec. V. Sweeping the gate voltages back to their original values (step 5) does not need to be done particularly slowly because when we open the central valve again, the system must have time to relax into the even-even parity ground state anyway. However, it is again favorable to perform this step adiabatically in other to avoid that the system is further excited (see discussion of step 4 in the fusion-rule protocol in Sec. VF

This, in sum, illustrates that the additional pumping steps in the current-readout scheme are in fact fast compared to the Majorana manipulation time scales and also usual charge sensing times (see Ref. [55]). The disadvantage is that the current-readout scheme only allows for an ensemble measurement. Moreover, the topological
Majorana case can be distinguished from nontopological Andreev states on the islands just by comparing with the predicted magnitude of the current $(I=e f)$. Trivial subgap states close to zero energy would lead in general to different, parameter-dependent but still nonzero pumping current since these states most probably lead to different probabilities for the even-even and odd-odd parity outcome.

\section{Appendix F: Effect of transitions into higher excited states}

In this Appendix, we argue that the adiabaticity criteria we derived in Secs. V. VI, and App. E are valid even if we account for the possibility of transitions into higher excited states. The criteria worked out there only account for transitions to the lowest accessible state while transitions into higher-lying excited states have been disregarded. However, they may be important, in principle, because there are many excited states and their cumulative effect might lead to stricter adiabaticity criteria than those derived in the foregoing section. Note that we do not take quasiparticle states into account here; we merely include the excited states within our Hamiltonian (4).

We argue in this Appendix that even if we sum condition (43) over all excited states, one can still satisfy the condition

$$
\sum_{k} g_{k} \ll \Delta t
$$

with the contributions

$$
g_{k}=\int_{C} d \boldsymbol{\lambda} \cdot\left|\frac{\left\langle\psi_{k}(\boldsymbol{\lambda})|\partial H(\boldsymbol{\lambda}) / \partial \boldsymbol{\lambda}| \psi_{0}(\boldsymbol{\lambda})\right\rangle}{\left[E_{k}(\boldsymbol{\lambda})-E_{0}(\boldsymbol{\lambda})\right]^{2}}\right|,
$$

without major corrections to the time scales estimated in Secs. V, VI. In all the steps we considered in these sections, we change only a single parameter $\lambda=E_{M}, E_{J, \alpha}$. Our analysis in the main part showed that one traverses (maximally) two regimes when changing $\lambda$ : the chargedominated regime, $\lambda \lesssim E_{C}$, and the tunneling / Josephson dominated regime, $\lambda \gtrsim E_{C}$. We next discuss these regimes separately.

\section{Charge-dominated part}

To make a conservative estimate for condition (F1) in the charge-dominated regime, we use that the eigenenergies of the Hamiltonian are roughly given by $E_{n_{L} n_{R}}=$ $E_{C}\left(n_{L}^{2}+n_{R}^{2}\right)$. Here, we label the states by the number of electrons $n_{L}$ and $n_{R}$ on the left and right island, respectively, and assume that the number of electrons in the ground state $\left|\psi_{0}(t)\right\rangle$ is close to $n_{L}=n_{R}=0$. In particular, we assumed here that the gatings $n_{g, L}=n_{g, R}=0$ but this assumption is uncritical because the quadratic increase of the energies with $n_{L}, n_{R}$ is also present for 
(a)

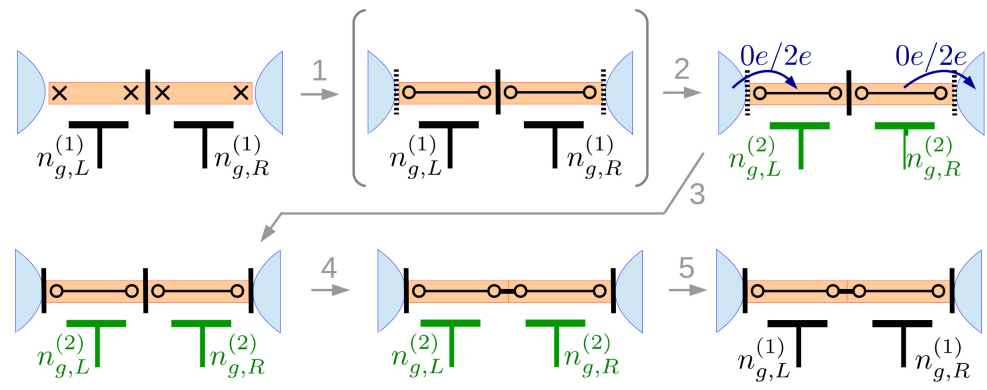

(b)

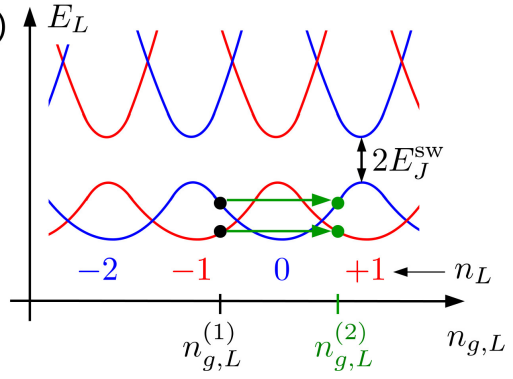

FIG. 12: Parity-dependent charge pumping. (a) Sketch of the steps of the pumping process. (b) Sketch of the energy spectrum for the left island as a function of the gating $n_{g, L}$. Sweeping the gating from $n_{g, L}^{(1)} \rightarrow n_{g, L}^{(2)}$ changes the charge on the island by 0 if the parity is even (blue) and by $2 e$ if the parity is odd (red). This works oppositely for the right island with $n_{g, R}^{(1)}=n_{g, L}^{(2)}$ and $n_{g, R}^{(2)}=n_{g, L}^{(1)}$. Note that the labeling of the charge states is not valid near the charge-degeneracy points, where states that differ by one Cooper pair mix with each other.

other gatings. The quadratic increase is important for the suppression of the effect of higher-lying states. Furthermore, the resulting energy gaps are larger when corrections due to the Majorana and / or Josephson couplings are included so that condition (F1) should be fulfilled even more easily.

$$
\sum_{k} g_{k} \leqslant g_{1} \times\left(\sum_{n_{L}, n_{R} \geqslant 1} \frac{1}{\left[n_{L}^{2}+n_{R}^{2}\right]^{2}}\right),
$$

with $g_{10}=\int_{\lambda_{\min }}^{\lambda_{\max }} d \lambda / E_{C}^{2}$. Replacing the sum by 1 , we would recover the time-scale estimate given in the main part 101. In writing down the above equation, we have also exploited that for all our estimates in Secs. V. VI, and App. E we never compute the matrix element $\left|\left\langle\psi_{k}|\partial H(t) / \partial \boldsymbol{\lambda}| \psi_{0}\right\rangle\right|$ (unless it is zero) but instead estimate it by its maximal value.

One can easily check that the sum on the right-hand side just gives a correction factor close to 1 : Using the inequality $n_{L}^{2}+n_{R}^{2} \geqslant 2 n_{L} n_{R}$, we get

$$
\begin{aligned}
\sum_{\left|n_{L}\right|,\left|n_{R}\right| \geqslant 1} \frac{1}{\left[n_{L}^{2}+n_{R}^{2}\right]^{2}} & \leqslant\left(\sum_{n_{L} \geqslant 1} \frac{1}{n_{L}^{2}}\right)\left(\sum_{n_{R} \geqslant 1} \frac{1}{n_{R}^{2}}\right) \\
& \approx 2.7,
\end{aligned}
$$

where we used $\sum_{n \geqslant 1} 1 / n^{2} \approx 1.64$. This shows that the adiabaticity criterion can indeed be verified by just considering the lowest excited state.

\section{Tunneling-dominated part}

Let us next turn to the tunneling-dominated regime. Here, the energy gaps of the excited states to the ground state read $E_{k}-E_{0}=k E_{10}$ with the gap $E_{10}=\sqrt{\alpha \lambda E_{C}}\left(\lambda=E_{M}, E_{J}\right)$ of the first excited state to the ground state ( $\alpha$ is a proportionality constant) [102. The transition matrix elements are given by $\left|\left\langle\psi_{k}(t)|\partial H / \partial \lambda| \psi_{0}(t)\right\rangle\right| \sim|\langle k|A| 0\rangle|$ with $A=\cos (\hat{\varphi})$ for the Josephson energy terms or $A=\cos (\hat{\varphi} / 2) i \hat{\gamma}_{2} \hat{\gamma}_{3}$ for the Majorana energy terms. These matrix elements are strongly suppressed with increasing $k$ since

$$
\left.\begin{array}{r}
|\langle k|\cos (\hat{\varphi} / 2)| 0\rangle| \\
|\langle k|\cos (\hat{\varphi})| 0\rangle|
\end{array}\right\}=\frac{c_{k}}{k !}\left|\left\langle k\left|\hat{\varphi}^{k}\right| 0\right\rangle\right| \propto \frac{1}{k !}
$$

with $c_{k}$ on the order of 1 or zero if $k$ is odd. One may therefore estimate

$$
g_{k} \sim g_{1} \frac{1}{k^{2} k !} .
$$

Using next that each of the plasmon states is maximally $2 k$-fold degenerate, we obtain

$$
\sum_{k} g_{k} \lesssim g_{0} \sum_{k \geqslant 1} k \times \frac{1}{k^{2} k !} \leqslant e g_{0},
$$

which again results in a correction factor of order 1 compared to the time-scale estimate derived for the lowest excited state ( $e$ in the above expression is the Euler number).

\section{Appendix G: Screening of charging energy by Majorana tunnel coupling}

In this Appendix, we discuss the screening of the charging energy of a wire segment when coupled to another nanowire segment through a Majorana coupling of the form (20). Let us concretely consider the situation when the left valve in the segmented wire structure in Fig. 1 is open $\left(E_{J, L} \gg E_{C}\right)$, while the right valve is closed $\left(E_{J, R}=0\right)$. The question is how the energy gap between the two local parity states on the right island $\left(p_{34}=0,1\right)$ changes when the Majorana coupling $E_{M}$ is increased by opening the central valve.

We employ for this purpose the following toy Hamiltonian (60), which only includes the parity degrees of 
freedom:

$$
H_{\mathrm{eff}}=i E_{M} \hat{\gamma}_{2} \hat{\gamma}_{3}+i \varepsilon_{P, R} \hat{\gamma}_{3} \hat{\gamma}_{4}
$$

We use here the notation introduced in Sec. III The above Hamiltonian crucially relies on the assumption $E_{J, L}, E_{M} \gg E_{C, R}$, in which both segments can be treated as being grounded. Otherwise the Cooper pair condensate can not be ignored and the full Hamiltonian including all charge states has to be used. Equation (G1) does thus not incorporate a term of the form $\sim i \varepsilon_{P, L} \hat{\gamma}_{1} \hat{\gamma}_{2}$ because the corresponding charging-induced energy splitting $\varepsilon_{P, L} \propto e^{-\sqrt{8 E_{J, L} / E_{C, 1}}}$ is much smaller than $E_{M}$ and $\varepsilon_{P, R}$ provided $E_{J, L} \gg E_{M}$. This leaves us first with the Coulomb-induced coupling $\varepsilon_{P, R}$ between MBS $\gamma_{3}$ and $\gamma_{4}$, which is in principle a function of all parameters of the model, in particular $E_{M}$ and $E_{J, L}$. Second, we have to account for the tunneling-induced coupling of MBS $\gamma_{2}$ and $\gamma_{3}$, where we neglected fluctuations in the phase difference of both islands contained in the fractional Josephson energy (4), i.e., the effect of the Cooper pair condensate. This works here in order to study the two lowest states, which exhibit no plasma oscillations.

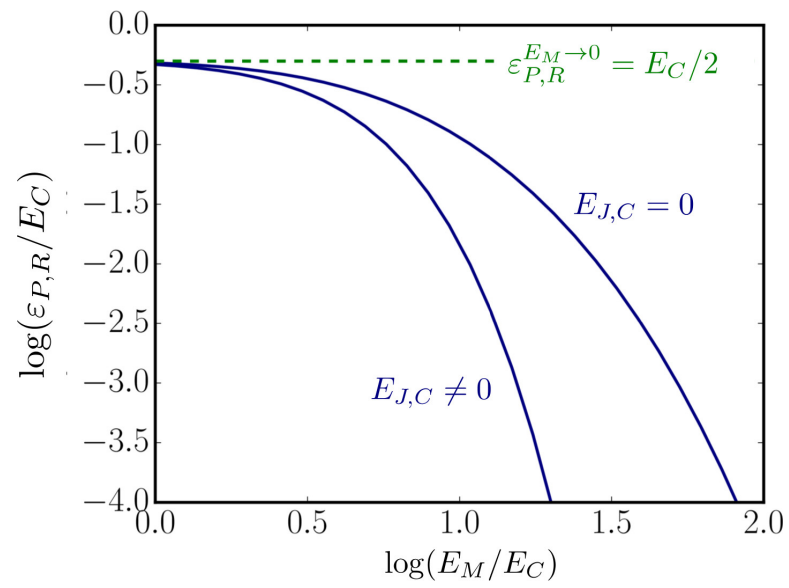

FIG. 13: Numerically computed energy gap $\varepsilon_{P, R}$, Eq. G6 (solid blue lines), between odd and even parity states on the right island as a function of the central Majorana coupling $E_{M}$. We also indicate the parity energy splitting $\varepsilon_{P, R}\left(E_{M} \rightarrow\right.$ $0)=E_{C, R} / 2$, corresponding to the two lowest charge states in the limit of vanishing Majorana coupling (green dashed). For the central Josephson coupling, we use $E_{J, C}=5 E_{M}^{2} / \Delta$ if nonzero as indicated and the other parameters are given by $E_{C, R}=E_{C, L}=E_{C}, n_{g, L}=n_{g, R}=0, E_{J, L}=100 E_{C}, E_{J, R}=$ $0, \Delta=100 E_{C}$.

The Hamiltonian (G1) admits two MBS at zero energy. One of them is $\gamma_{1}$ (since it is decoupled from the other MBS) and the other one is

$$
\hat{\gamma}_{0}=a \hat{\gamma}_{2}+b \hat{\gamma}_{4}=\frac{\varepsilon_{P, R} \hat{\gamma}_{2}+E_{M} \hat{\gamma}_{4}}{\sqrt{\varepsilon_{P, R}^{2}+E_{M}^{2}}} .
$$

The ground state is thus degenerate with respect to the occupation of the fermionic mode $\hat{f}_{10}=\left(\hat{\gamma}_{1}+i \hat{\gamma}_{0}\right) / 2$ with number operator $\hat{n}_{10}=\left(1+i \hat{\gamma}_{1} \hat{\gamma}_{0}\right) / 2$. The expectation values $\left\langle\hat{n}_{10}\right\rangle=0,1$ characterize the total parity of the two islands. The ground state with even total parity, $\left\langle\hat{n}_{10}\right\rangle=0$, satisfies

$$
-1=\left\langle i \hat{\gamma}_{1} \hat{\gamma}_{0}\right\rangle=\frac{\varepsilon_{P, R}\left\langle i \hat{\gamma}_{1} \hat{\gamma}_{2}\right\rangle+E_{M}\left\langle i \hat{\gamma}_{1} \hat{\gamma}_{4}\right\rangle}{\sqrt{\varepsilon_{P, R}^{2}+E_{M}^{2}}} .
$$

To extract $\varepsilon_{P, R}$ as a function of the parameters, we compute expectation values on the right hand side numerically. The expectation value $\left\langle i \hat{\gamma}_{1} \hat{\gamma}_{2}\right\rangle$ is connected to that of the operator introduced by Eq. 46):

$$
\left\langle i \hat{\gamma}_{1} \hat{\gamma}_{2}\right\rangle=\left\langle\hat{P}_{o o}\right\rangle-\left\langle\hat{P}_{e e}\right\rangle .
$$

To obtain $\left\langle i \hat{\gamma}_{1} \hat{\gamma}_{4}\right\rangle$, we can exploit the fact that the Hilbert space of the effective Hamiltonian (G1) is twodimensional when restricted to the two lowest states with even total parity. In this subspace, we can define Pauli matrices $\hat{\sigma}_{z}=i \hat{\gamma}_{1} \hat{\gamma}_{2}=i \hat{\gamma}_{3} \hat{\gamma}_{4}$ and $\hat{\sigma}_{x}=i \hat{\gamma}_{2} \hat{\gamma}_{3}=i \hat{\gamma}_{1} \hat{\gamma}_{4}$ (but the equalities are valid only in this subspace, not in general). For a pure state, the corresponding Bloch vector must have length 1 and this implies $\left\langle\hat{\sigma}_{x}\right\rangle^{2}+\left\langle\hat{\sigma}_{z}\right\rangle^{2}=1$ and thus

$$
\left\langle i \hat{\gamma}_{1} \hat{\gamma}_{4}\right\rangle=-\sqrt{1-\left\langle i \hat{\gamma}_{1} \hat{\gamma}_{2}\right\rangle^{2}} .
$$

(The sign follows from the fact that the energy of the ground state must be minimal.) Equation (G3) can now be solved for $\varepsilon_{P, R}$,

$$
\varepsilon_{P, R}=\frac{E_{M}}{\sqrt{1 /\left\langle i \hat{\gamma}_{1} \hat{\gamma}_{2}\right\rangle^{2}-1}},
$$

implying for the coefficients in Eq. G2):

$$
a=\left\langle i \hat{\gamma}_{1} \hat{\gamma}_{4}\right\rangle, \quad b=\sqrt{1-\left\langle i \hat{\gamma}_{1} \hat{\gamma}_{2}\right\rangle^{2}} .
$$

The result for the coefficient $a$ has been shown and discussed in Sec. VIA2 We briefly discuss here the dependence of the parity splitting $\varepsilon_{P, R}$ on $E_{M}$, which is shown in Fig. 13. Indeed, $\varepsilon_{P, R}$ is not constant as a function of $E_{M}$ but becomes exponentially small for $E_{M} \gg E_{C}$. This shows that the charging energy on the right island can be effectively suppressed by opening the central junction. Importantly, this does not require the system to be tuned into the single island regime $E_{M} \gg$ $E_{J, L}$ (cf. App. D 2): In the regime $E_{M} \ll E_{J, L}$, the left island functions similar to a bulk superconductor since its Josephson plasma ground state has a distribution in charge space that is much broader than that of the right island.

In Fig. 13 we also show results down to $E_{M} \sim E_{C, R}$ even though Eq. (G1) is strictly speaking not valid in this regime. What we intend to illustrate is that our 
procedure nevertheless approaches the correct value for $\varepsilon_{P, R}$ in the limit $E_{M} / E_{C, R} \rightarrow 0$, which is simply half the energy difference between the two lowest charge states $n_{R}=0,1$ (green dashed line).
We thus have argued that gate-controlled transfer of MBS between two nanowire segments is possible by tuning just a single valve - the one connecting the two nanowire segments.
[1] B. A. Bernevig and T. L. Hughes, Topological insulators and topological superconductors (Princeton University Press, 2013).

[2] A. Y. Kitaev, Sov. Phys.-Uspeki 44, 131 (2001).

[3] J. Alicea, Rep. Prog. Phys. 75, 076501 (2012).

[4] M. Leijnse and K. Flensberg, Semicond. Sci. Technol. 27, 124003 (2012).

[5] C. W. J. Beenakker, Annu. Rev. Con. Mat. Phys. 4, 113 (2013).

[6] T. D. Stanescu and S. Tewari, J. Phys.: Condens. Matter 25, 233201 (2013).

[7] S. Elliott and M. Franz, arXiv:1403.4976 (unpublished).

[8] C. Nayak, M. Freedman, and S. Das Sarma, npj Quantum Information 1, 15001 (2015).

[9] E. C. Rowell and Z. Wang, arXiv preprint arXiv:1508.04793 (2015).

[10] C. Nayak, S. H. Simon, A. Stern, M. Freedman, and S. Das Sarma, Rev. Mod. Phys. 80, 1083 (2008).

[11] A. Y. Kitaev, Ann. Phys. 303, 2 (2003).

[12] F. Hassler, arXiv preprint arXiv:1404.0897 (2014).

[13] D. A. Ivanov, Phys. Rev. Lett. 86, 268 (2001).

[14] C. Nayak, S. H. Simon, A. Stern, M. Freedman, and S. Das Sarma, Rev. Mod. Phys. 80, 1083 (2008).

[15] N. Read and D. Green, Phys. Rev. B 61, 10267 (2000).

[16] S. Das Sarma, C. Nayak, and S. Tewari, Phys. Rev. B 73, 220502 (2006).

[17] L. Fu and C. L. Kane, Phys. Rev. Lett. 100, 096407 (2008).

[18] Q.-F. Liang, Z. Wang, and X. Hu, Europhys. Lett. 99, 50004 (2012).

[19] S.-P. Lee, J. Alicea, and G. Refael (in preparation).

[20] L. Fu and C. L. Kane, Phys. Rev. B 79, 161408(R) (2009).

[21] J. D. Sau, R. M. Lutchyn, S. Tewari, and S. Das Sarma, Phys. Rev. Lett. 104, 040502 (2010).

[22] T.-P. Choy, J. M. Edge, A. R. Akhmerov, and C. W. J. Beenakker, Phys. Rev. B 84, 195442 (2011).

[23] S. Nadj-Perge, I. K. Drozdov, B. A. Bernevig, and A. Yazdani, Phys. Rev. B 88, 020407 (2013).

[24] R. M. Lutchyn, J. D. Sau, and S. Das Sarma, Phys. Rev. Lett. 105, 077001 (2010).

[25] Y. Oreg, G. Refael, and F. von Oppen, Phys. Rev. Lett. 105, 177002 (2010).

[26] J. Alicea, Phys. Rev. B 81, 125318 (2010).

[27] L. P. Rokhinson, X. Liu, and J. K. Furdyna, Nat. Phys. 8, 795 (2012).

[28] S. Nadj-Perge, I. K. Drozdov, J. Li, H. Chen, S. Jeon, J. Seo, A. H. MacDonald, B. A. Bernevig, and A. Yazdani, Science 346, 602 (2014).

[29] A. D. K. Finck, D. J. Van Harlingen, P. K. Mohseni, K. Jung, and X. Li, Phys. Rev. Lett. 110, 126406 (2013).

[30] V. Mourik, K. Zuo, S. M. Frolov, S. R. Plissard, E. P. A. M. Bakkers, and L. P. Kouwenhoven, Science 336, 1003 (2012).
[31] A. Das, Y. Ronen, Y. Most, Y. Oreg, M. Heiblum, and H. Shtrikman, Nat. Phys. 8, 887 (2012).

[32] M. T. Deng, C. L. Yu, G. Y. Huang, M. Larsson, P. Caroff, and H. Q. Xu, Nano Lett. 12, 6414 (2012).

[33] H. O. H. Churchill, V. Fatemi, K. Grove-Rasmussen, M. T. Deng, P. Caroff, H. Q. Xu, and C. M. Marcus, Phys. Rev. B 87, 241401 (2013).

[34] J. Alicea, Y. Oreg, G. Refael, F. von Oppen, and M. P. A. Fisher, Nat. Phys. 7, 412 (2011).

[35] P. Bonderson, Phys. Rev. B 87, 035113 (2013).

[36] D. J. Clarke, J. D. Sau, and S. Tewari, Phys. Rev. B 84, 035120 (2011).

[37] B. I. Halperin, Y. Oreg, A. Stern, G. Refael, J. Alicea, and F. von Oppen, Phys. Rev. B 85, 144501 (2012).

[38] A. Romito, J. Alicea, G. Refael, and F. von Oppen, Phys. Rev. B 85, 020502 (2012).

[39] M. Burrello, B. van Heck, and A. R. Akhmerov, Phys. Rev. A 87, 022343 (2013).

[40] J. Li, T. Neupert, B. A. Bernevig, and A. Yazdani, arXiv preprint arXiv:1404.4058 (2014).

[41] J. D. Sau, D. J. Clarke, and S. Tewari, Phys. Rev. B 84, 094505 (2011).

[42] T. Hyart, B. van Heck, I. C. Fulga, M. Burrello, A. R. Akhmerov, and C. W. J. Beenakker, Phys. Rev. B 88, 035121 (2013).

[43] Ö. Gül, D. J. van Woerkom, I. van Weperen, D. Car, S. R. Plissard, E. P. A. M. Bakkers, and L. P. Kouwenhoven, Nanotechnology 26, 215202 (2015).

[44] P. Krogstrup, N. L. B. Ziino, W. Chang, S. M. Albrecht, M. H. Madsen, E. Johnson, J. Nygård, C. M. Marcus, and T. S. Jespersen, Nature Materials 14, 400 (2015).

[45] W. Chang, S. M. Albrecht, T. S. Jespersen, F. Kuemmeth, P. Krogstrup, J. Nygård, and C. M. Marcus, Nature Nanotechnology 10, 232 (2015).

[46] K. A. Dick, K. Deppert, M. W. Larsson, T. Mårtensson, W. Seifert, L. R. Wallenberg, and L. Samuelson, Nat. Mater. 3, 380 (2004).

[47] S. R. Plissard, I. van Weperen, D. Car, M. A. Verheijen, G. W. G. Immink, J. Kammhuber, L. J. Cornelissen, D. B. Szombati, A. Geresdi, S. M. Frolov, et al., Nature Nanotechnology 8, 859 (2013).

[48] Y. Makhlin, G. Schön, and A. Shnirman, Reviews of modern physics 73, 357 (2001).

[49] M. H. Devoret, A. Wallraff, and J. Martinis, arXiv preprint cond-mat/0411174 (2004).

[50] A. Cottet, Implementation of a quantum bit in a superconducting circuit (Doctoral thesis, Université Paris VI, Paris (France), 2002).

[51] J. Koch, T. M. Yu, J. Gambetta, A. A. Houck, D. I. Schuster, J. Majer, A. Blais, M. H. Devoret, S. M. Girvin, and R. J. Schoelkopf, Phys. Rev. A 76, 042319 (2007).

[52] F. Hassler, A. R. Akhmerov, C.-Y. Hou, and C. W. J. Beenakker, New Journal of Physics 12, 125002 (2010).

[53] B. van Heck, A. R. Akhmerov, F. Hassler, M. Burrello, 
and C. W. J. Beenakker, New Journal of Physics 14 035019 (2012).

[54] F. Hassler, A. R. Akhmerov, and C. W. J. Beenakker, New Journal of Physics 13, 095004 (2011).

[55] D. Aasen, M. Hell, R. V. Mishmash, A. Higginbotham, J. Danon, M. Leijnse, T. S. Jespersen, J. A. Folk, C. M. Marcus, K. Flensberg, et al. (2015), unpublished.

[56] G. de Lange, B. van Heck, A. Bruno, D. J. van Woerkom, A. Geresdi, S. R. Plissard, E. P. A. M. Bakkers, A. R. Akhmerov, and L. DiCarlo, Phys. Rev. Lett. 115, 127002 (2015).

[57] T. W. Larsen, K. D. Petersson, F. Kuemmeth, T. S. Jespersen, P. Krogstrup, J. Nygård, and C. M. Marcus, Phys. Rev. Lett. 115, 127001 (2015).

[58] Y.-P. Shim and C. Tahan (2015), unpublished, 1507.07923.

[59] M. Cheng, V. Galitski, and S. Das Sarma, Phys. Rev. B 84, 104529 (2011).

[60] M. S. Scheurer and A. Shnirman, Phys. Rev. B 88, 064515 (2013).

[61] T. Karzig, G. Refael, and F. von Oppen, Phys. Rev. X 3, 041017 (2013).

[62] T. Karzig, A. Rahmani, F. von Oppen, and G. Refael, Phys. Rev. B 91, 201404 (2015).

[63] T. Karzig, F. Pientka, G. Refael, and F. von Oppen, Phys. Rev. B 91, 201102 (2015).

[64] C. Knapp, M. Zaletel, D. E. Liu, M. Cheng, P. Bonderson, and C. Nayak, arXiv preprint condmat/1601.05790 (2016).

[65] A. P. Higginbotham, S. M. Albrecht, G. Kirsanskas, W. Chang, F. Kuemmeth, P. Krogstrup, T. S. Jespersen, J. Nygard, K. Flensberg, and C. M. Marcus, Nat. Phys. p. 1017 (2015).

[66] L. Fu, Phys. Rev. Lett. 104, 056402 (2010).

[67] L. W. Molenkamp, K. Flensberg, and M. Kemerink, Phys. Rev. Lett. 75, 4282 (1995).

[68] H. Schoeller and G. Schön, Phys. Rev. B 50, 18436 (1994).

[69] Y. V. Nazarov and Y. M. Blanter, Quantum transport: introduction to nanoscience (Cambridge University Press, 2009)

[70] W. G. van der Wiel, S. D. Franceschi, J. M. Elzerman, T. Fujisawa, S. Tarucha, and L. P. Kouwenhoven, Rev. Mod. Phys. 75, 1 (2003).

[71] L. Schiff, Quantum mechanics (New York: McGrawHill, 1968).

[72] T. Fujisawa, D. G. Austing, Y. Tokura, Y. Hirayama, and S. Tarucha, Nature 419, 278 (2002).

[73] J. R. Petta, A. C. Johnson, C. M. Marcus, M. P. Hanson, and A. C. Gossard, Phys. Rev. Lett. 93, 186802 (2004).

[74] G. Catelani, R. J. Schoelkopf, M. H. Devoret, and L. I. Glazman, Physical Review B 84, 064517 (2011).

[75] D. Ristè, C. Bultink, M. Tiggelman, R. Schouten, K. Lehnert, and L. DiCarlo, Nature communications 4, 1913 (2013).

[76] G. Catelani, Physical Review B 89, 094522 (2014).

[77] C. Wang, Y. Y. Gao, I. M. Pop, U. Vool, C. Axline, T. Brecht, R. W. Heeres, L. Frunzio, M. H. Devoret, G. Catelani, et al., Nature communications 5 (2014).

[78] D. Rainis and D. Loss, Phys. Rev. B 85, 174533 (2012).

[79] D. J. van Woerkom, A. Geresdi, and L. P. Kouwenhoven, Nature Physics 11, 547 (2015).

[80] M. Barkeshli and J. D. Sau, arXiv preprint
arXiv:1509.07135 (2015)

[81] K. Flensberg, Phys. Rev. B 82, 180516 (2010).

[82] A. Zazunov, A. L. Yeyati, and R. Egger, Phys. Rev. B 84, 165440 (2011).

[83] M. Tinkham, Introduction to superconductivity (Courier Corporation, 2012).

[84] K. Flensberg, Phys. Rev. B 82, 180516 (2010).

[85] S. Das Sarma, J. D. Sau, and T. D. Stanescu, Phys. Rev. B 86, 220506 (2012).

[86] A. V. Pronin, M. Dressel, A. Pimenov, A. Loidl, I. V. Roshchin, and L. H. Greene, Phys. Rev. B 57, 14416 (1998).

[87] T. B. Boykin and G. Klimeck, Eur. J. Phys. 25, 503 (2004).

[88] This holds irrespective of the specific form of the Josephson coupling. The bulk superconductors can only transfer electrons in (Cooper) pairs to the island, which does not change the fermion parity of the islands.

[89] We use a different sign factor in the exponentials of Eqs. 13 and (14) as compared to Ref. 66 so that the displacement relation 9 becomes compatible with the commutation relation (8).

[90] A state $|\psi\rangle$ has even (odd) total parity here if the difference of the number of fermionic modes occupied for $|\psi\rangle$ and the reference state $\left|n_{L}, n_{R}\right\rangle=|0,0\rangle$ is even (odd). Note that $n_{L}$ and $n_{R}$ are just defined up to arbitrary offset electron numbers whose parities are not specified.

[91] The Hamiltonian for a Cooper pair box [Eq. (5) for one island] reads in electron number states: $H=$ $\sum_{n}\left[E_{C}|n\rangle\langle n|+E_{J} / 2(|n+2\rangle\langle n|+| n-2\rangle\langle n|)\right]$. The Hamiltonian $H_{\Delta}$ follows by replacing $n \rightarrow \Delta n, E_{C} \rightarrow E_{C} / 2$, $E_{J} \rightarrow E_{M}$ ), and changing the number of electrons by 1 instead of 2 in the Hamiltonian.

[92] We assume $n_{g, L}=n_{g, R}=0$; for other gatings the energy can be reduced and the order of the levels may reverse.

[93] The ground state becomes two-fold degenerate. We checked numerically that the splitting between the corresponding states $\left|1_{14} 0_{23}\right\rangle$ and $\left|0_{14} 0_{23}\right\rangle$ is given by $\varepsilon_{P}$ [Eq. [37] ] and therefore exponentially small in

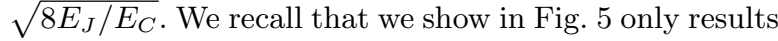
for even total parity (including $\left|0_{14} 0_{23}\right\rangle$ ) since transitions into states of odd total parity (including $\left|1_{14} 0_{23}\right\rangle$ ) are forbidden and therefore irrelevant for the time-scale considerations.

[94] The matrix element $A_{k}=\left|\left\langle\psi_{k}\left|\partial H / \partial E_{J}\right| \psi_{0}\right\rangle\right|=$ $\left|\left\langle\psi_{k}\left|\cos \left(\hat{\varphi}_{1}\right)+\cos \left(\hat{\varphi}_{2}\right)\right| \psi_{0}\right\rangle\right|$ in Eq. 45 can actually become much smaller than 1 in the limit $E_{J} \gg E_{M} \gg$ $E_{C}$. If one approximates $\left|\psi_{k}\right\rangle \approx\left|k_{1}, k_{2} ; p_{14}, p_{23}\right\rangle$ in that limit, one first finds $A_{k_{1}=1, k_{2}=0}=A_{k_{1}=0, k_{2}=1}=$ $A_{k_{1}=1, k_{2}=1}=0$, that is, transitions into the first excited states vanish. The reason for this is that $\cos \left(\hat{\varphi}_{\alpha}\right)$ contains only an even power of $\hat{\varphi}_{\alpha}=\hat{a}_{\alpha}+\hat{a}_{\alpha}^{\dagger}$ and therefore a product of an even number of ladder operators $\hat{a}_{\alpha}, \hat{a}_{\alpha}^{\dagger}$, each changing $k_{\alpha}$ by $-1,+1$, respectively. However, taking the next excited states in condition (47) would just increase the gap by a factor of $\sqrt{2}$. What is more important is that the matrix element is suppressed as $A_{k_{2}=2, k_{1}=0}, A_{k_{2}=0, k_{1}=2} \propto \sqrt{E_{C} / E_{J}}$. Inserting this into Eq. 45 , integrand in Eq. 47 scales as $\propto 1 / \sqrt{E_{J}}$ instead of $\propto 1 / E_{J}$. This would remove logarithmic dependence in the condition (48). However, to give a solid quantitative prediction, one should also include cor- 
rections for deviations of $\left|\psi_{k}\right\rangle$ from $\left|k_{1}, k_{2} ; p_{14}, p_{23}\right\rangle$ for $E_{J} \sim E_{C}$. In the same order, higher-harmonic corrections to the Josephson energy might become relevant. We limit our discussion therefore to our conservative estimate.

[95] Note that one of the two charge states is an excited state of the system (here $\left|n_{L}=-1, n_{R}=+1\right\rangle$ ) but transitions to the ground state (here $\left|n_{L}=0, n_{L}=0\right\rangle$ ) are not possible because the central valve is closed.

[96] Note that relaxation rates in the other steps of the protocol should be slower: Josephson plasma oscillations in nontopological nanowire setups relax typically on the microsecond scale 57.

[97] We assumed that the number $n_{e}^{\prime}$ of occupied unpaired modes excluding the zero mode is even. Then the parity of the island $p_{12}=n_{0}$ equals the occupation $p_{12}$ of the zero-energy mode and the unitary transformation A5 contributes an irrelevant $2 \pi$-periodic phase factor $e^{i n_{e}^{\prime} \varphi / 2}$ to the wave function (since it does not change). If $n_{e}^{\prime}$ is odd, we could proceed in the same way by connecting the parity via $p_{12}=1+n_{0}$ to the occupation $n_{0}$ of the zero-energy mode.
[98] The phases across the junctions with larger couplings $E_{T}>E_{\min }$ are more strongly locked and phase fluctuations are more strongly suppressed as compared to the phase difference across the junction with coupling energy $E_{\min }$.

[99] This is correct for real $E_{M}$ assumed throughout the paper. For complex $E_{M}=e^{i \kappa}\left|E_{M}\right|$, the eigenstates are given by $\left(\left|k, 0_{12} 0_{34}\right\rangle \pm e^{i \kappa}\left|k, 1_{12} 1_{34}\right\rangle\right) / \sqrt{2}$.

[100] This holds both when $E_{J, \alpha}^{\mathrm{sw}} \sim E_{C}$, because $E_{1}-E_{0} \geqslant$ $2 E_{J, \alpha}^{\mathrm{sw}}$ as Fig. 12(b) illustrates, but also when $E_{J, \alpha}^{\mathrm{sw}} \gg$ $E_{C}$ when $E_{1}-E_{0} \approx \sqrt{8 E_{J, \alpha}^{\mathrm{sw}} E_{C}}$.

[101] Note that $g_{10}$ refers here to an excited state with odd total parity $\left(n_{L}=1, n_{R}=0\right.$ or $\left.n_{L}=0, n_{R}=1\right)$, which cannot be reached from the ground state. However, in the main part we use $E_{1}-E_{0}=2 E_{C} \sim E_{C}$, which is why $g_{10}=\int_{\lambda_{\min }}^{\lambda_{\max }} d \lambda / E_{C}^{2}$ reproduces the result from the main part.

[102] Note that this formula does not hold in step 2 of the fusion-rule protocol discussed in Sec. VD however, we can use $E_{k 0} \geqslant k \sqrt{\alpha E_{C} E_{M}}$ there and therefore apply our considerations in this section. 